\title{
Textes et textes de loi sur la gestion des ressources naturelles au Burkina Faso
}

\section{Z. Henri-Noël Bouda}
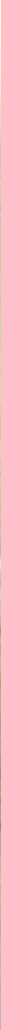



\section{Textes et textes de loi sur la gestion des ressources naturelles au Burkina Faso}

Z. Henri-Noël Bouda 
Sources des photos de la couverture:

- Daniel TIVEAU (CIFOR/BRAO) pour la photo de fond et la quatrième photo (éléphant)

- Direction de communication de l'Assemblée nationale, pour la photo présentant la façade de l'Assemblée nationale et la photo du Président de l'Assemblée nationale, plantant un arbre.

Bureau Régional Afrique Occidentale

Adresse de bureau : Situé à l'intérieur du CNRST, face à l'Hôpital Yalgado

Ouédraogo - Ouagadougou

Adresse postale : 06 BP 9478 Ouagadougou 06, Burkina Faso

Tel.: +22650304742 Fax : +22650302930

Site web: www.cifor.cgiar.org E-mail: d.tiveau@cgiar.org 


\section{Table des matières}

Sigles et abréviations $\quad \mathrm{v}$

Remerciements vii

Résumé vii

Introduction 1

I Les différentes ressources naturelles 3

1.1. Définition de RN 3

1.2. Les différentes $\mathrm{RN}$ existantes 3

1.3. Répartition des compétences concernant les $\mathrm{RN} 4$

II. Les principaux textes et textes de loi sur la GRN 6

2.1. Les textes sur le foncier 6

2.1.1. La Réforme Agraire Foncière 6

2.1.2. La Politique nationale de sécurisation foncière en milieu rural 8

2.1.3. Autres textes sur le foncier 9

2.2. Les textes sur les ressources forestières 10

2.2.1. De 1900 à l'indépendance 10

2.2.2. De l'indépendance à nos jours 11

2.2.3. Autres documents d'orientation concernant les ressources forestières 13

2.3. Les documents de référence concernant la ressource $\begin{array}{ll}\text { naturelle eau } & 15\end{array}$

2.4. Les textes sur les ressources fauniques 18

2.5. Les textes sur les ressources pastorales 19

2.6. Les textes sur les ressources halieutiques et piscicoles 20

2.7. Les textes sur les ressources minières et les carrières 21 
2.8. Autres documents de politiques prenant en compte les ressources naturelles

2.8.1. Lettre de Politique de Développement Rural

Décentralisé

2.8.2. Cadre Stratégique de Lutte contre la Pauvreté

2.8.3. Stratégie de Développement Rural à l'horizon $2015 \quad 23$

2.8.4. Stratégie nationale de gestion des feux en milieu rural 24

2.8.5. Le Programme d'action national d'adaptation à la variabilité et aux changements climatiques

2.8.6. La politique nationale d'aménagement du territoire

III. Les conventions locales pour la GRN 27

3.1. La définition d'une convention locale $\quad 27$

3.2. La place des conventions locales $\quad 27$

3.3. Le texte sur les conventions locales 28

3.4. Les signataires des conventions locales 28

3.5. Les phases d'élaboration d'une convention locale 29

$\begin{array}{lr}\text { Conclusion } & 30\end{array}$

Références $\quad 31$

Annexes $\quad 33$

iv $\quad$ Textes et textes de loi sur la gestion des ressources naturelles au Burkina Faso 


\section{Sigles et abréviations}

\begin{tabular}{|c|c|}
\hline ASDI & $\begin{array}{l}\text { Agence Suédoise de coopération pour le Développement } \\
\text { International }\end{array}$ \\
\hline CGCT & Code Général des Collectivités Territoriales \\
\hline CIFOR & Center for International Forestry Research \\
\hline CNEau & Conseil National de l’Eau \\
\hline CNSFMR & Comité National de Sécurisation Foncière en Milieu Rural \\
\hline CSLP & Cadre Stratégique de Lutte contre la Pauvreté \\
\hline CT & Collectivité Territoriale \\
\hline CVD & Comités Villageois de Développement \\
\hline CVGT & Commission Villageoise de Gestion des Terroirs \\
\hline DGFROP & $\begin{array}{l}\text { Direction Générale du Foncier Rural et des Organisations } \\
\text { Paysannes }\end{array}$ \\
\hline FAO & $\begin{array}{l}\text { Food and Agriculture Organization (Organisation des Nations } \\
\text { Unies pour l'Alimentation et l'Agriculture) }\end{array}$ \\
\hline FEM & Fonds pour l'Environnement Mondial \\
\hline GGAOF & Gouverneur-Général de l'Afrique de l'Ouest Française \\
\hline GRN & Gestion des Ressources Naturelles \\
\hline LIPDHD & $\begin{array}{l}\text { Lettre d'Intention de Politique de Développement Humain } \\
\text { Durable }\end{array}$ \\
\hline LPDRD & Lettre de Politique de Développement Rural Décentralisé \\
\hline MAHRH & $\begin{array}{l}\text { Ministère de l'Agriculture de l'Hydraulique et des Ressources } \\
\text { Halieutiques }\end{array}$ \\
\hline MATD & $\begin{array}{l}\text { Ministère de l'Administration Territoriale et de la } \\
\text { Décentralisation }\end{array}$ \\
\hline MECV & Ministère de l'Environnement et du Cadre de Vie \\
\hline MEDEV & Ministère de l'Economie et du Développement \\
\hline MET & Ministere de l'Environnement et du Tourisme \\
\hline MFB & Ministère des Finances et du Budget \\
\hline MID & Ministère des Infrastructures et du Développement \\
\hline ONG & Organisation Non Gouvernementale \\
\hline $\begin{array}{l}\text { PADSEA- } \\
\text { II }\end{array}$ & $\begin{array}{l}\text { Programme d'Appui Danois au Secteur Eau et Assainissement, } \\
\text { phase II }\end{array}$ \\
\hline PAGIRE & Plan d'Action de Gestion Intégrée des Ressources en Eau \\
\hline PAN/LCD & Plan d'Action National de Lutte Contre la Désertification \\
\hline PANA & $\begin{array}{l}\text { Programme d'action National d'Adaptation à la variabilité et } \\
\text { aux changements climatiques }\end{array}$ \\
\hline PANE & Plan d'Action National pour l'Environnement \\
\hline PFN & La Politique Forestière Nationale \\
\hline$M$ & Premier Ministère \\
\hline
\end{tabular}


PNAF Programme National d'Aménagement des Forêts

PNAT Politique Nationale d'Aménagement du Territoire

PNFV Programme National de Foresterie Villageoise

PNGRF Programme National de Gestion des Ressources Forestières au Burkina Faso

PNGT Programme National de Gestion des Terroirs

PNSFMR Politique Nationale de Sécurisation Foncière en Milieu Rural

PNUD Programme des Nations Unies pour le Développement

PPTE Pays Pauvres Très Endettés

RAF Réorganisation Agraire et foncière

RIGRN Règles Internes de Gestion des Ressources Naturelles

RN Ressources Naturelles

SDAU Schéma Directeur d'Aménagement et d'Urbanisme

SDPS Services Départementaux du Plan et de la Statistique

SDR Stratégie de Développement Rural

SNP Système National de Planification

SPAN/CB Stratégie et le Plan d'Action National sur la Conservation de la Biodiversité

SPAN/CC Stratégie et le Plan d'Action National sur les Changements Climatiques 


\section{Remerciements}

La réalisation de ce travail a été possible grâce au financement de l'Agence Suédoise de coopération pour le Développement International (ASDI) dans le cadre du projet forêts sèches de CIFOR. Nous tenons à exprimer à cette agence notre reconnaissance pour le soutien combien inestimable.

Nos remerciements vont également à :

- Son Excellence Monsieur Roch Marc Christian KABORE, Président de l'Assemblée National du Burkina Faso et à tous ses collaborateurs, qui ont accepté mettre à notre disposition les différents textes officiels.

- Au Secrétaire Général du Gouvernement et du Conseil des Ministres du Burkina Faso, et ses collaborateurs, pour nous avoir autorisé la copie des différents textes parus dans le Journal Officiel du Burkina Faso. Monsieur Romain August BAMBARA, Directeur de communication de l'Assemblée nationale, pour les photos mises à notre disposition.

- L'équipe régionale du CIFOR en Afrique de l'Ouest, notamment Daniel TIVEAU et Mathurin ZIDA, respectivement Coordonnateur Régional du CIFOR pour l'Afrique de l'Ouest et Chef de Projet Forêts Sèches, pour l'intérêt qu'ils ont porté au travail et pour s'être investis personnellement pour sa réalisation.

- Monsieur Bala Wenceslas SANOU, Consultant indépendant au bureau d'étude YIRIWA-CONSULT, pour ses conseils, ses commentaires et les corrections apportées au document. 


\section{Résumé}

La gestion durable des ressources naturelles, notamment dans les pays du Sahel, a toujours soulevé des problèmes dont celui de l'inadaptation des législations nationales y relatives. Le processus d'élaboration et de mise en œuvre de ces législations est à l'image des conditions politiques, socio-économiques et culturelles des pays en question.

Le Burkina Faso, pour sa part, a connu plusieurs formes de régimes politiques et, de ce fait, capitalise un certain nombre d'expériences de réformes législatives et réglementaires dans le secteur des forêts et des ressources naturelles. La renaissance démocratique du Burkina Faso suite à l'adoption de la constitution de 1991 a permis la restauration de l'Assemblée nationale à l'issue des élections législatives de 1992. Depuis, plusieurs textes juridiques sur l'existence, le fonctionnement et la vie de la nation et de ses structures ont été adoptés par l'assemblée. Au nombre de ceux-ci, on compte une dizaine de textes relatifs à la gestion des ressources naturelles.

Lobjectif de ce travail est de faire une compilation des principaux textes législatifs et réglementaires relatifs à la gestion, l'exploitation, la protection et la conservation des ressources naturelles au Burkina Faso. Après avoir donné un contour à la notion de ressources naturelles, ce document présentera les principaux textes des secteurs suivants :

- Le foncier

- Les ressources forestières

- La ressource naturelle eau

- Les ressources fauniques

- Les ressources pastorales

- Les ressources halieutiques et piscicoles

- Les ressources minières et les carrières

Dans le contexte de la décentralisation, les conventions locales jouent un rôle important dans la gestion décentralisées des ressources naturelles. Aussi le dernier chapitre traite-t-il de la question des conventions locales au Burkina Faso.

Mot clés : Burkina Faso, Gestion des ressources naturelles, Législation, Textes et lois. 


\section{Introduction}

La constitution burkinabè fait de la protection de l'environnement un devoir primordial. En effet dès son préambule, il annonce: «...conscient de la nécessité absolue de protéger l'environnement; approuvons et adoptons la présente Constitution dont le présent préambule fait partie intégrante». L'article 29 stipule : "Le droit à un environnement sain est reconnu; la protection, la défense et la promotion de l'environnement sont un devoir pour tous.»

Par ailleurs, les $\mathrm{RN}$ constituent la première source potentielle de revenu propre de la plupart des communes rurales au Burkina. Cette potentialité reste toutefois encore peu (voire pas) valorisée par les collectivités territoriales pour des raisons diverses dont la méconnaissance des possibilités offertes à travers le cadre juridique, réglementaire et les documents d'orientation politique. Tant pour les collectivités territoriales que toute autre personne physique ou morale, la connaissance des textes régissant la gestion et l'exploitation des $\mathrm{RN}$ constitue un préalable à toute intervention dans le domaine.

Pour le monde de la recherche et du développement notamment, l'ignorance des textes constitue également un blocage pour une intervention (action de développement ou de recherche).

Ce fascicule tient alors lieu de répertoire, bien que non exhaustif, des principaux textes qui régissent l'exploitation et la gestion des RN au Burkina Faso.

Nous nous efforcerons de faire un récapitulatif des textes en vigueur, avec un regard sur ceux qui sont antérieurs. Sans prétendre apporter une expertise de juriste sur ces textes, nous essayerons de donner des informations sur leur application sur le terrain. 



\section{Les différentes ressources}

\section{naturelles}

\subsection{Définition de RN}

Selon le dictionnaire «Le Petit Larousse Illustré» (2000), une ressource naturelle se définit comme étant «l'ensemble des potentialités qu'offre le milieu physique, notamment dans les domaines énergétique, minier ou forestien. Dans notre cas, nous adopterons une définition pragmatique de la notion des RN. Nous dirons que cette expression désigne les différents produits que l'Homme tire du milieu naturel pour sa survie ou pour des raisons mercantiles.

\subsection{Les différentes $\mathrm{RN}$ existantes}

La notion de $\mathrm{RN}$ regroupe différents produits émanant de plusieurs domaines qui ont fait l'objet de textes spécifiques au Burkina :

- Le domaine du foncier. La terre, support naturel des différentes ressources naturelles, est elle-même considérée comme ressource naturelle. Le foncier correspond aux rapports des hommes à cette ressource de base.

- Le domaine forestier avec le bois, les racines, les écorces, les fibres, les résines, les gommes, les feuilles, les fleurs, les fruits et le sous bois du couvert arboré ;

- Le domaine faunique avec les animaux sauvages ;

- Le domaine "eau" avec les fleuves, les rivières, les marigots, les "boulis", les mares et les nappes phréatiques ; 
- Le domaine pastoral avec les pâturages, les points d'abreuvement, les espaces naturels traversés lors des déplacements ;

- Le domaine halieutique avec les poissons et les plantes aquatiques ;

- Le domaine des mines (substances minérales) et carrières (sable, graviers, latérite, moellons, etc.)

La gestion, l'exploitation, l'utilisation ou l'accès à tous les produits et sous produits de ces différents domaines sont soumis à une réglementation à travers différents textes que nous évoquerons dans ce document.

\subsection{Répartition des compétences concernant les RN}

Conformément à la loi 055-2004/AN du 21/12/2004 portant Code général des collectivités territoriales, les CT peuvent assumer des compétences dans les domaines que l'Etat leur transfert. Le transfert de compétences s'effectue suivant le principe de la subsidiarité et celui de la progressivité. Les domaines de compétence que l'Etat transfert aux CT sont au nombre de onze (11). Dans le tableau 1, nous nous limiterons aux compétences relatives à la gestion des ressources naturelles (GRN).

Toujours conformément à la même loi, tout transfert de compétences doit être accompagné du transfert des moyens et des ressources nécessaires à l'exercice normal de ladite compétence.

- les compétences sont transférées avec tout ou partie des services correspondants ;

- le transfert est constaté par un décret de dévolution pris en conseil de ministre.

Les CT ont à leur tour des obligations dans l'exercice des compétences transférées. Ces obligations concourent à la bonne gouvernance locale :

- obligation de bien gérer ;

- obligation de rendre compte à la population dans un objectif de transparence ;

- obligation de rendre compte à la tutelle 
Tableau 1 : Les domaines de compétence à transférer aux CT

\begin{tabular}{|c|c|c|}
\hline Domaines & $\begin{array}{l}\text { Compétences exercées } \\
\text { exclusivement par la CT }\end{array}$ & $\begin{array}{l}\text { Compétences exercées en partage avec l'Etat } \\
\text { et d'autres personnes morales }\end{array}$ \\
\hline $\begin{array}{l}\text { Aménagement du } \\
\text { territoire et gestion } \\
\text { du domaine foncier }\end{array}$ & $\begin{array}{l}\text { - La gestion et l'utilisation du } \\
\text { domaine foncier propre des } \\
\text { CT comprenant des parties du } \\
\text { domaine foncier national cédé à } \\
\text { titre de propriété }\end{array}$ & $\begin{array}{l}\text { - La gestion et l'utilisation des parties du } \\
\text { domaine foncier national et du domaine privé } \\
\text { de l'Etat situés dans les ressorts territoriaux } \\
\text { des CT. } \\
\text { - avis sur le schéma d'aménagement; } \\
\text { - participation à la gestion des terres du } \\
\text { domaine foncier national situé dans les } \\
\text { ressorts territoriaux des CT; } \\
\text { En plus des compétences ci-dessus, les communes } \\
\text { rurales partagent la compétence suivante avec } \\
\text { l'Etat: } \\
\text { - participation à l'élaboration du schéma } \\
\text { d'aménagement de l'espace de production et } \\
\text { de conservation; }\end{array}$ \\
\hline $\begin{array}{l}\text { Gestion des } \\
\text { ressources naturelles }\end{array}$ & 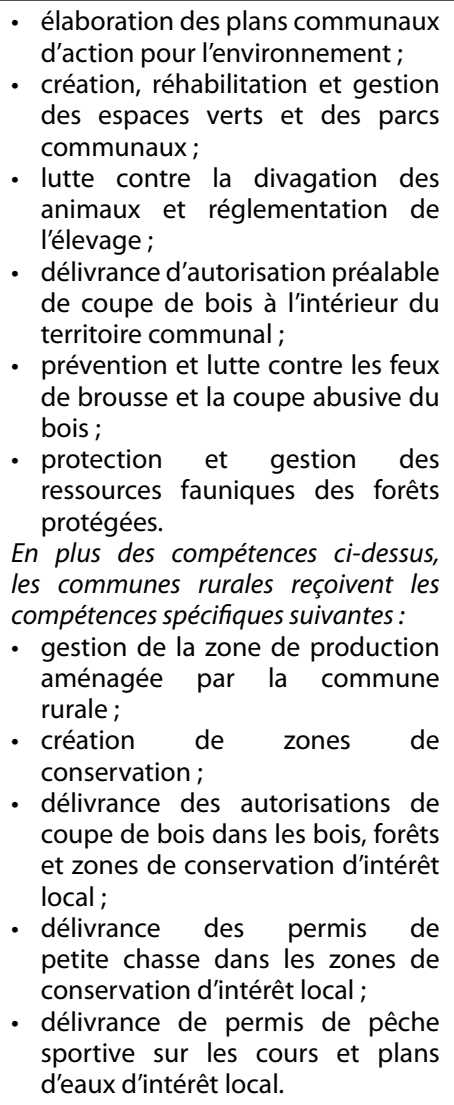 & $\begin{array}{l}\text { - participation à la protection et à la gestion } \\
\text { des ressources en eau souterraine, en eau de } \\
\text { surface et des ressources halieutiques; } \\
\text { - participation à la conservation et à la gestion } \\
\text { des ressources naturelles renouvelables } \\
\text { d'intérêt régional ou national; } \\
\text { - participation à la protection et à la gestion des } \\
\text { ressources fauniques des forêts classées; } \\
\text { En plus des compétences ci-dessus, les communes } \\
\text { rurales partagent les compétences suivantes avec } \\
\text { l'Etat et/ou d'autres personnes: } \\
\text { - participation à la gestion de la zone de } \\
\text { production aménagée par d'autres personnes } \\
\text { morales sur le territoire de la commune } \\
\text { rurale; } \\
\text { participation à la protection et à la gestion des } \\
\text { ressources naturelles situées sur le territoire } \\
\text { de la commune rurale; } \\
\text { participation à la protection et à la gestion } \\
\text { des forêts naturelles, de la faune sauvage, } \\
\text { des ressources en eau et des ressources } \\
\text { halieutiques situées sur les territoires de la } \\
\text { commune rurale. }\end{array}$ \\
\hline Eau et électricité & $\begin{array}{l}\text { - production et distribution de l'eau } \\
\text { potable; } \\
\text { - réalisation et gestion de puits, de } \\
\text { forages et de bornes fontaines }\end{array}$ & $\begin{array}{l}\text { - avis sur le schéma Directeur } \\
\text { d'approvisionnement en eau; } \\
\text { - participation à l'élaboration du schéma } \\
\text { régional d'électrification. }\end{array}$ \\
\hline
\end{tabular}

Source : loi 055-2004/AN du 21/12/2004 portant Code général des collectivités territoriales 


\section{Les principaux textes et textes de loi sur la GRN}

La renaissance démocratique au Burkina en 1991, a permis, à la suite des élections législatives de 1992, la restauration de l'Assemblée nationale. Depuis, plusieurs textes juridiques sur l'existence, le fonctionnement et la vie de la nation et de ses structures ont été adoptés par l'assemblée. De cette longue liste, on dénombre une dizaine de textes relatifs à la GRN. Nous ferons mention ici des plus importants adoptés par l'assemblée nationale ou non.

\subsection{Les textes sur le foncier}

\subsubsection{La Réforme Agraire Foncière}

Après l'indépendance de la Haute Volta en 1960, l'une des premières mesures de l'Etat était la «domanialisation» de certaines terres pour un meilleur contrôle de la gestion des ressources. Cette domanialisation a entrainé la cohabitation de deux systèmes (droit moderne et droit coutumier) sur la gestion des terres. Une opposition du légal (droit moderne) au légitime (droit coutumier) est donc née, créant donc un contraste entre la théorie (loi moderne) et la pratique (droit coutumier). Ce droit coutumier était même de fait accepté par l'administration au niveau local. La RAF, initiée en 1985 sous la Révolution, est venu lever l'équivoque qui semblait faire jour. La RAF conférait alors la propriété exclusive du patrimoine foncier à l'Etat. Ainsi, l'article 4 souligne que "le domaine foncier national est de plein droit propriété de l'Etat" Par Domaine 
Foncier National, il fallait comprendre entre autres les terres détenues en vertu des coutumes, ce qui sous entend le démantèlement partiel de la chefferie traditionnelle (Matthias B. et al. 2000). Une critique imputée à la RAF porte sur son principe de concentration du pouvoir décisionnel entre les mains de l'Etat et ses démembrements. Les communautés locales n'ont presque pas de pouvoir de décision sur le domaine foncier. Désormais, les terres devaient être attribuées par des autorités compétentes de l'Etat, sous forme d'un droit de jouissance, avec obligation de mise en valeur effective de ces terres. La RAF s'inscrit alors dans une vision du rôle fort de l'Etat et s'appuie sur la logique de l'aménagement du territoire suivant les contraintes préalablement établies par des techniciens. Après la période stricte de l'Etat d'exception, la RAF a connu plusieurs relectures en 1994 et 1996.

Avec les ajustements ultérieurs, la possibilité de constitution de domaines privés a été introduite. A cet effet, l'article 5 fait mention de cession à titre de propriété privée aux personnes physiques ou morales de certaines terres du domaine foncier. Par ailleurs des domaines de responsabilité sont prévus pour les CT, notamment dans le décret $\mathrm{n}^{\circ}$ 97-054/PRES/PM/MEF portant conditions et modalités d'application de la RAF. Les ouvertures faites portent sur les points suivants:

- avis divers sur les instruments d'aménagement (schémas national, régional, provincial d'aménagement du territoire, schéma d'aménagement et d'urbanisme, lotissement);

- présidence des commissions d'attributions des terres à usage d'habitation;

- participation au contrôle sur l'utilisation de l'eau (articles 239, 258, 267) ;

- droit d'usage, protection des forêts et de la faune (articles 232, 307).

Le Programme National de Gestion des Terroirs (PNGT), un des produits de la RAF a été mis en place en 1987 pour encourager un développement rural participatif et de planification à la base, donnant un certain pouvoir de décision aux villageois quant à l'affectation des terres et leur gestion. A ce titre, des Commissions Villageoises de Gestion des Terroirs (CVGT) ont été mises en place dans un certain nombre de villages pour assurer les fonctions d'attribution et de gestion des terres villageoises. Cependant, les fonctions qui leur ont été assignées par les textes d'orientation sur la gestion foncière n'étaient guère assurées. Les structures traditionnelles (chef de terre, chef de village) ont conservé un rôle influent. Ainsi, les CVGT sont restées très peu fonctionnelles car les difficultés liées à l'articulation entre les institutions traditionnelles préexistantes et effectives, et ces institutions imposées de l'extérieur (l'Etat), sont restées pendantes (Matthias B. et al. 2000) 


\subsubsection{La Politique nationale de sécurisation foncière en milieu rural}

Puisque la RAF n'a pas connue une application effective, le «flou» qu'elle était sensée relever persiste. Du reste, son application ne garantissait pas non plus une résolution des problèmes connus depuis la période coloniale. La situation actuellement semble d'ailleurs évoluer au pire.

En effet, au Burkina Faso, les éléments d'une véritable situation de crise foncière se mettent progressivement en place dans le milieu rural (MAHRH 2007) :

- compétition accrue et conflictuelle entre acteurs pour le contrôle et l'exploitation des terres ;

- poursuite et même intensification dans certaines régions, des migrations agricoles et des transhumances pastorales;

- multiplication et aggravation des conflits entre acteurs ruraux à l'occasion de la mise en valeur des terres et de l'exploitation des ressources naturelles;

- développement d'un processus de concentration des terres entre les mains d'entrepreneurs ruraux dénommés agro businessmen ou " nouveaux acteurs»;

- faible efficacité des mécanismes juridiques et institutionnels de gestion foncière et de gestion des conflits en milieu rural.

Pour apporter des réponses efficaces et durables au problème de la sécurisation foncière des acteurs ruraux, le gouvernement du Burkina Faso a décidé d'entreprendre l'élaboration d'un document de politique nationale de sécurisation foncière en milieu rural (PNSFMR).

L'option d'élaborer un tel document s'appuie sur plusieurs considérations (MAHRH 2007) :

- la complexité des questions à résoudre nécessite une démarche préalable rigoureuse d'établissement d'un diagnostic des problèmes fonciers majeurs et une approche concertée d'élaboration des solutions à mettre en œuvre ;

- l'expérience récente a montré que les relectures successives et techniques de la législation foncière en vigueur (RAF) n'avaient pas permis de trouver des réponses appropriées à la question de la sécurisation foncière rurale ;

- des options de politique foncière claires et faisant consensus au plan national sont un préalable à l'élaboration avec succès d'une future législation foncière rurale adaptée et effective.

\begin{tabular}{l|l} 
8 & Textes et textes de loi sur la gestion des ressources naturelles au Burkina Faso
\end{tabular} 
Dans le cadre de l'élaboration de la PNSFMR, la mise en œuvre d'un processus de dialogue politique national tranche avec les expériences passées de relectures de la RAF, essentiellement techniques et confinées aux mains de spécialistes.

La PNSFMR vise à assurer à l'ensemble des acteurs ruraux, l'accès équitable au foncier, la garantie de leurs investissements et la gestion efficace des différends fonciers, afin de contribuer à la réduction de la pauvreté, à la consolidation de la paix sociale et à la réalisation du développement durable. Cinq objectifs spécifiques sont définis pour la PNSFMR (MAHRH 2007) :

- garantir le droit d'accès légitime de l'ensemble des acteurs ruraux au foncier, dans une dynamique de développement rural durable, de lutte contre la pauvreté et de promotion de l'équité et de la légalité ;

- contribuer à l'amélioration de la prévention et du règlement des conflits liés au foncier et à la gestion des ressources naturelles ;

- contribuer à créer les bases de la viabilité et du développement des CT par la mise à leur disposition de ressources foncières propres et des outils efficaces de gestion ;

- accroître l'efficacité des services de l'Etat et des CT dans l'offre d'un service public adapté et effectif de sécurisation foncière en milieu rural ;

- promouvoir la participation effective des acteurs de base et de la société civile à la mise en œuvre, au suivi et à l'évaluation de la PNSFMR.

Le document de PNSFMR fera l'objet d'une loi relative à la sécurisation foncière en milieu rural, à soumettre à l'assemblée nationale pour adoption.

\subsubsection{Autres textes sur le foncier}

Le foncier a toujours constitué un dossier délicat au niveau du parlement. En effet, comme le souligne le document de Politique nationale de sécurisation foncière en milieu rural, outre l'opposition entre la loi moderne et le coutumier, il y a que la situation foncière n'est pas homogène sur l'ensemble du territoire national; elle est fortement diversifiée selon les principales zones socio foncières ${ }^{1}$. Pendant que certaines zones se caractérisent par une rareté des ressources naturelles (zone Nord, zone du Plateau central), d'autres bénéficient de ressources naturelles relativement abondantes (zones Sud, zone Sud Ouest). Du point de vue démographique, certaines zones connaissent une forte concentration de population (zone du Plateau central) pendant que la pression démographique est faible dans d'autres (zones Est, Sud Ouest). Ceci

\footnotetext{
Les 7 zones socio foncières ci-après ont été identifiées : zones nord ; zone du plateau central ; zone est ; zone sud ; zone sud-ouest ; zone de vielle colonisation agricole ; zone de front pionnier.
} 
crée une diversité de pratiques foncières que le législateur trouve tant de peine à harmoniser dans un texte réglementaire.

Outre les 2 textes majeurs ci-dessus, l'Assemblée nationale, a adopté les textes légiférant la gestion des terres, notamment le domaine urbain et périurbain, à usage d'habitation ou d'activités diverses (industrielles, artisanales, etc.) :

N'étant donc pas intimement lié à la GRN ces lois ne feront pas l'objet de commentaire dans ce document.

\subsection{Les textes sur les ressources forestières}

Dans le contexte de ce document, nous entendons par ressources forestières le bois, les racines, les écorces, les fibres, les résines, les gommes, les feuilles, les fleurs, les fruits et le sous bois du couvert arboré. En somme, il s'agit des produits forestiers ligneux et non ligneux, le règne animal étant exclu.

Avant d'abordé les textes fondamentaux régissant la gestion et l'exploitation de ces ressources, faisons un peu d'histoire sur l'évolution des textes.

\subsubsection{De 1900 à l'indépendance}

En juillet 1900, le service colonial de l'agriculture et des forêts a promulgué la première législation forestière de l'Afrique de l'Ouest Française. Le code a institué des permis, des concessions, des espèces et des zones protégées, et des droits usufructuaires. Il a placé les forêts directement sous le contrôle de l'État colonial, avec le Gouverneur-Général et ses délégués contrôlant l'attribution des permis et des concessions. Les droits d'exploitation commerciale étaient gérés par la branche exécutive du gouvernement - pas le Service Forestier. Le dégagement de forêt et la coupe des espèces à bois de "haute qualité» ne pouvaient être conduits qu'avec la permission du commandant de chaque Cercle. Le code a également établi des droits d'usage au profit des populations locales, tout en ouvrant la porte à la repression (Ribot. 2001) : "Dans les bois et les forêts du Domaine [des colonies d'Afrique occidentale françaises] non concédés $\grave{a}$ des individus, les indigènes continueront à exercer les droits d'usage (jardinage, coupe ordinaire de bois, pâturage, chasse, etc.) dont ils jouissent actuellement. Si leurs comportements de l'abus des droits ci-dessus énoncées compromettent la richesse forestière du domaine forestier, le Gouverneur-Général prendra par décret, toutes les mesures d'interdiction et de sauvegarde nécessaires."(GGAOF 1916, art. 23 in Ribot. 2001).

Ainsi ce premier code forestier d'Afrique occidentale a placé des droits commerciaux sous le contrôle de l'État tout en reléguant aux populations rurales

10 Textes et textes de loi sur la gestion des ressources naturelles au Burkina Faso 
les produits forestiers considérés comme n'ayant aucune valeur marchande. En effet, aux populations indigènes, seulement un droit de collecte a été donné pour ces produits pour lesquels le Service Forestier n'avait pas d'intérêt. En outre, ces droits d'usage pouvaient disparaitre à tout moment si les forestiers et les administrateurs affectaient de telles régions boisées aux utilisateurs commerciaux (Ribot 1999). Ils étaient simplement des utilisateurs de seconde classe, au gré des droits primordiaux des utilisateurs commerciaux. Le but d'une telle politique était de protéger des espèces commercialement valables contre l'épuisement par les utilisateurs commerciaux et indigènes, par l'intermédiaire de l'attribution des permis et des concessions et de la perception d'impôts de forêt par des agents de gouvernement. Dans la période coloniale, ces permis et concessions ont été habituellement assignées aux négociants européens et urbains (Ribot 2001).

Au cours des cent dernières années, il y a eu seulement de légères variations de cette approche. Une loi du février 1908 a établi un impôt sur le bois de chauffage et le charbon de bois et a exigé des permis pour leur transport (en plus des permis de production déjà existants). Un nouveau code forestier de l'Afrique de l'ouest française (couvrant la Mauritanie, le Sénégal, la Guinée, le Soudan, le Niger, la Haute-Volta et le Dahomey) a été ratifié le 4 juillet 1935 et tient à ce jour comme modèle de loi forestière pour toute l'Afrique de l'ouest francophone (Ribot 2001). Ce code était beaucoup plus affiné que celui de 1900. Le nouveau code a réaffirmé la propriété de l'Etat sur les forêts et tous les produits forestiers. Ce code qui renforce la propriété de l'Etat sur les forêts a exigé des permis pour toute l'exploitation commerciale de la forêt, a présenté de diverses restrictions d'utilisation, et a spécifié des pénalités pour les infractions. Par ailleurs, de même qu'auparavant, des terres non exploitées comme terres agricoles (y compris les forêts) pouvaient être confisquées sans compensation.

Le décret d'application de 1937 a spécifié les droits des indigènes de faire des canoës et puis a énuméré les nombreuses espèces d'arbre protégées contre leur utilisation. Ces espèces protégées pouvaient seulement être coupées quand un permis de coupe avait été obtenu pour l'installation des superficies agricoles.

\subsubsection{De l'indépendance à nos jours}

Après l'indépendance, le code de 1935 est demeuré en vigueur dans la plupart des pays francophones d'Afrique occidentale jusqu'aux années 80. A partir de la fin des années 80 , beaucoup de pays sahéliens ont réécrit leur code forestier, qualifiant leur approche de participative et "fondée sur la communauté». 
Au Burkina Faso, le code forestier a été adopté par l'assemblée nationale en 1997, puis promulgué la même année. Il constitue la loi de base relative aux ressources forestières, fauniques et halieutiques.

Les premiers articles définissent clairement son objectif et son but. En effet, l'article 1 annonce: "Le présent Code a pour objet de fixer, conformément à la Politique forestière nationale, l'ensemble des principes fondamentaux relatif $\grave{a}$ la conservation et à la gestion des ressources naturelles forestières, fauniques et halieutiques», et l'article 2 précise : "Il vise en particulier à établir une articulation harmonieuse entre la nécessaire protection de ces ressources et la satisfaction des besoins économiques, culturels et sociaux de la population». Il ne s'agit donc pas d'une protection absolue et exclusive, mais d'une protection qui prend en compte la satisfaction des besoins de la population. La notion de la participation est intégrée dès l'article 3: "Le Code définit les conditions favorables au développement performant des sous-secteurs des forêts, de la faune et des pêches tout en assurant la promotion des principes de gestion participative et durable des ressources concernées».

Le code forestier précise que l'ensemble des formations forestières du pays est réparti en forêts publiques (article 16) et en forêts privées (article 33). Les forêts publiques sont réparties entre le patrimoine de l'Etat et le patrimoine des CT (article 17.)

Ce code donne une place de choix à une politique forestière nationale dont il définit les options en son l'article 7 : «La politique forestière nationale est fondée sur les principales options fondamentales suivantes:

- la conservation de la diversité biologique;

- la valorisation des ressources forestières, fauniques et halieutiques pour le développement économique et l'amélioration du cadre de vie;

- la génération d'emplois et des revenus au profit de la population;

- la participation et la responsabilisation effective de la population dans la conception, l'exécution, le suivi et l'évaluation des activités forestières, notamment à travers la gestion décentralisée des ressources naturelles.»

La politique forestière nationale (PFN) qui a vu le jour en 1995 et qui traite des sous - secteurs forêts, faune et pêches, confirme le rôle central des services forestiers de l'Etat dans la préservation des ressources forestières considérées comme éléments du patrimoine national, tout en reconnaissant la participation des populations à la gestion et à l'exploitation des forêts comme principe de bonne gouvernance des ressources forestières.

12 Textes et textes de loi sur la gestion des ressources naturelles au Burkina Faso 
La PFN vise essentiellement les objectifs suivants (MECV 1998) :

- "Traduire la place et le rôle des sous-secteurs forêts, faune et pêches et de préciser les options du gouvernement à travers les axes prioritaires de développement qui sy rapportent, en fonction des missions du Ministère de tutelle;

- Rationaliser la gestion des ressources des trois sous-secteurs forêts, faune et pêches. Cette rationalisation signifie, avant tout, une mise en cohérence et en synergie des interventions dans ces trois sous-secteurs avec de nettes améliorations ;

- Conférer une base conceptuelle pour l'élaboration de la législation afférente à la gestion de ces trois sous-secteurs ;

- Constituer un outil de négociation et un cadre de référence quant aux concertations avec les partenaires au développement pour la coordination et l'harmonisation des interventions menées dans les trois sous-secteurs."

\subsubsection{Autres documents d'orientation concernant les ressources forestières}

L'environnement est l'un des secteurs qui a connu le plus de programmes et projets. «L'amour national» pour l'environnement s'est accru depuis les années 80 avec le mouvement révolutionnaire. Revenons sur quelques points importants de ces grands programmes.

Le lancement des Trois luttes le 22 avril 1985 (lutte contre les feux de brousse, lutte contre la divagation des animaux et lutte contre la coupe abusive du bois) a marqué un tournant majeur dans l'aménagement des forêts. Ce mot d'ordre politique a accéléré de fait, à partir de 1986, le démarrage de l'aménagement participatif des forêts qui a fait l'objet en 1981 d'une politique circonscrite aux forêts classées, grâce au projet Aménagement et exploitation des forêts pour le ravitaillement de Ouagadougou en bois de feu (Projet PNUD/FAO/ $\mathrm{BKF} / 85 / 011$ ) financé par le PNUD à la requête du Gouvernement pour appuyer l'application de la Lutte contre la coupe anarchique du bois (MECV. 2007)

En 1984 naquit le Programme National de Foresterie Villageoise (PNFV) qui sera relu en 1991. Son approche stratégique qui fût l'approche participative. Les expériences capitalisées en matière de foresterie villageoise et d'aménagement forestier ont permis entre autre l'adoption par le Gouvernement, en 1996 d'un Programme National d'Aménagement des Forêts (PNAF). Le PNFV et le PNAF feront l'objet d'une fusion en 2007 pour donner le programme national de gestion des ressources forestières 2006-2015. Le nouveau programme définit quatre axes d'orientations : 
- "La capitalisation des acquis et l'amélioration des connaissances en matière de gestion durable des ressources forestières.

- L'accroissement et la diversification des productions forestières;

- Le renforcement des capacités des acteurs ;

- Le renforcement du cadre politique, juridique et institutionnel en matière de gestion durable des ressources forestières."

Le Plan d'action national pour l'environnement (PANE) élaboré en 1991 vise pour objectif principal la recherche d'un équilibre socio-écologique et socio-économique susceptible de contribuer à l'autosuffisance et à la sécurité alimentaire et d'offrir les meilleures conditions de vie aux populations. A moyen et long termes, le PANE vise particulièrement à (MET 1991) :

- "Maîtriser les pressions sur le milieu naturel;

- Favoriser la régénération des RN et à protéger la biodiversité;

- Améliorer le cadre et les conditions de vie des populations;

- Amorcer le processus d'un développement durable.»

Le Plan d'Action National de Lutte Contre la Désertification (PAN/LCD) adopté par le Gouvernement en 2000 est un instrument de mise en œuvre de la convention de lutte contre la désertification, ratifiée par le Burkina Faso. Il se fixe comme objectif global de contribuer à l'instauration d'un développement durable du pays par le renforcement des capacités des autorités locales ainsi que d'assurer la participation active des populations, des collectivités et des groupes locaux dans les actions de lutte contre la désertification et d'atténuation des effets de la sécheresse.

Notons pour clore ce chapitre, le plan d'action de mise en œuvre des reformes institutionnelles et juridique pour la décentralisation dans le secteur forestier (MECV 2006). La mise en œuvre du plan d'action s'appuie sur des principes directeurs conformes à ceux du Cadre Stratégique de Lutte contre la Pauvreté (CSLP) et de la Stratégie de Développement Rurale (SDR) qui sont :

- la promotion de la bonne gouvernance ;

- le développement du capital humain ;

- le renforcement du processus de décentralisation ;

- la prise en compte de l'approche genre ;

- le renforcement du partenariat entre l'Etat et les autres acteurs.

14 Textes et textes de loi sur la gestion des ressources naturelles au Burkina Faso 


\subsection{Les documents de référence concernant la ressource naturelle eau}

Tout comme le domaine forestier national se compose des forêts publiques et des forêts privées, la ressource eau au niveau national comprend les eaux publiques naturelles ou artificielles et les eaux privées composées des eaux d'étang, de mares ou de fossés creusés sur des fonds privés. Notons tout d'abord l'élaboration et l'adoption d'instruments pour la gestion prospective et durable des ressources en eau et de l'assainissement ces dernières années :

- la Stratégie nationale de l'assainissement (1996) ;

- le Document de politique et stratégies en matière d'eau (1998) ;

- le Document cadre sur la gestion et la maintenance des points d'eau en milieux rural et semi urbain et son décret d'application (2000);

- la Loi d'orientation sur la gestion de l'eau (2001);

- le Plan d'action pour la gestion intégrée des ressources en eau-PAGIRE (2003);

- la Politique nationale de développement durable de l'agriculture irriguée et le Plan d'action y relatif pour l'horizon 2015 (2004);

- le Lancement de la “Biennale eau 2004-2005" pour contribuer à la réduction significative de la pauvreté (2004) ;

- le Plan d'action 2004-2008 de l'ONEA pour l'approvisionnement en eau potable des centres urbains (2004) :

- le Programme d'Appui Danois au Secteur Eau et Assainissement, phase II (PADSEA II) pour la période 2005 - 2009.

- la Stratégie nationale de l'approvisionnement en eau potable (en étude);

Comme textes de référence pour la gestion de la ressource naturelle eau, retenons le Code de l'environnement, le code forestier, la RAF, la loi $n^{\circ} 002-$ 2001/AN portant loi d'orientation relative à la gestion de l'eau et le PAGIRE

Le code de l'environnement traite de la pollution des eaux dans son chapitre six. Ce chapitre traite notamment des droits de rejet de déchets dans les eaux.

Comprises comme élément des ressources forestières, les ressources eaux sont traitées dans le code forestier. Ce code porte sur l'administration forestière la charge de protection des eaux, en son article 48 : «Sur l'ensemble du domaine forestier, l'administration chargée des forêts est habilitée à prendre toutes les mesures nécessaires par les conditions spécifiques du milieu et notamment la fixation des sols en pente, la protection des terres et des ouvrages contre l'action érosive, la 
conservation des espèces rares et des biotopes fragiles, la protection des sources et des cours d'eau».

La RAF dans son chapitre III, section I (articles 71 à 83) traite de la gestion de l'eau. Elle dispose d'abord que: "Au sens de la présente loi, constituent des eaux domaniales ou eaux publiques, toutes les ressources en eau ainsi que les constructions et aménagements hydrauliques appartenant aux personnes morales de droit public ou réalisés dans un but d'intérêt général" (article 71), puis précise les différentes composantes de la ressource eau à son article 72 : "Les ressources en eau comprennent les eaux superficielles, souterraines et atmosphériques tels que fleuves, rivières, lacs, étangs, mares et leurs dépendances légales, nappes souterraines et nuages dans les limites de l'espace national»

La loi $\mathrm{n}^{\circ}$ 002-2001/AN portant loi d'orientation relative a la gestion de l'eau, stipule en son article 5 que "L'eau est un élément du patrimoine commun de la Nation. Elle fait partie du domaine public», et précise à son article 6 que «... le domaine public de l'eau comprend l'eau dans ses divers états physiques et situations géomorphologiques ainsi que les ouvrages publics affectés ou nécessaires à sa gestion. $Y$ sont inclus à ce titre :

- les cours d'eau;

- les lacs naturels ou artificiels, les étangs, les mares et d'une manière générale, les étendues d'eau;

- les espaces où la présence de l'eau, sans être permanente, est régulière et empêche ou conditionne directement l'exploitation à des fins agricoles;

- les eaux souterraines;

- l'eau atmosphérique;

- les sources, puits, forages, abreuvoirs et autres points d'eau affectés à l'usage du public ou à un service public ainsi que leurs périmètres de protection immédiate, délimités en application de l'article 34, alinéa 1 ;

- les digues, les barrages, les chaussées, les écluses et leurs dépendances ou ouvrages annexes;

- les canaux d'irrigation, d'assainissement et de drainage ;

- les aqueducs, les canalisations, les dérivations et les conduites d'eau; les réservoirs, les stations de traitement d'eau potable, les stations d'épuration des eaux usées et, d'une manière générale, les ouvrages hydrauliques affectés à l'usage du public ou à un service public ainsi que les installations et les terrains qui en dépendent»

Cette loi de référence en gestion de l'eau au Burkina désigne clairement le gestionnaire principale de la ressource : "Le ministère chargé de l'eau est le garant institutionnel de la gestion intégrée des ressources en eau» (article 13).

16 Textes et textes de loi sur la gestion des ressources naturelles au Burkina Faso 
Toutefois, la loi tient compte de la décentralisation et stipule que «Les décisions relatives à la gestion de l'eau sont prises par les autorités locales, dans le cadre de la collectivité ou de la circonscription administrative dont le champ territorial de compétences est le plus restreint, sous réserve qu'aucune considération d'intérêt général ou liée à la nécessité de satisfaire dans les meilleures conditions les besoins en eau de toute nature ne s'y oppose» (article 14). Les autorités locales peuvent même recevoir des compétences en matière de gestion d'eau dans leur territoire. En effet, l'article 43 fait cette ouverture :»Dans tout ou partie du territoire d'une collectivité territoriale, l'Etat peut déléguer à celle-ci, aux conditions qu'il définit conformément à la loi, certaines de ses compétences relatives à l'utilisation de l'eau. Cette délégation concerne la gestion du service public de distribution d'eau potable, ou des utilisations de l'eau à des fins agricoles, aquacoles, pastorales, industrielles, touristiques ou de production d'énergie. Elle peut porter également sur l'assainissement, entendu comme le traitement et l'évacuation des eaux usées et, le cas échéant, l'évacuation des eaux pluviale.»

Le Plan d'Action pour la Gestion Intégrée des Ressources en Eau (PAGIRE) adopté en 2001 a pour objectif général de : "Contribuer à la mise en ouvre d'une gestion intégrée des ressources en eau du pays, adaptée au contexte national, conforme aux orientations définies par le Gouvernement burkinabè et respectant les principes reconnus au plan international en matière de gestion durable et écologiquement rationnelle des ressources en eau».

Fondé sur le document de politique et stratégies en matière d'eau et organisé autour de huit domaines d'actions², le PAGIRE qui court de 2003 à 2015 intègre les grandes orientations suivantes :

- "Recentrer les missions de l'Etat;

- Mettre en place le Conseil National de l'Eau (CNEau) en tant qu'organe national pour une gestion concertée de l'eau associant l'Etat, les collectivités locales, le secteur privé et la société civile;

- Construire de nouveaux espaces de gestion sur la base des bassins hydrographiques en tant que circonscriptions spécifiques appropriées pour la planification et la gestion de l'eau;

- Renforcer les capacités d'intervention des collectivités locales, du secteur privé et de la société civile dans le domaine de l'eau;

- Développer et renforcer les ressources humaines.»

\footnotetext{
${ }^{2}$ Les huit domaines d'action sont : «environnement habilitant»; "système d'information sur l'eau»; "procédures de gestion»; «recherche/développement»; «ressources humaines»; «information, éducation, sensibilisation, plaidoyer»; «cadre institutionnel» et «mesures d'urgence»
} 


\subsection{Les textes sur les ressources fauniques}

Les ressources fauniques constituent l'une des 3 ressources dont le code forestier définit les conditions favorables de gestion. Les ressources fauniques font donc partie du patrimoine national et conservées, protégées et gérées comme telles. Le titre II (article 71 à 162) du code forestier est consacré à la faune qui est défini comme "l'ensemble des animaux sauvages, vivant en liberté dans leur milieu naturel ou maintenus en captivité, à l'exception des poissons, des mollusques et des crustacés» (article 71).

En tant que patrimoine national, la gestion de la faune concerne en premier l'Etat. Toutefois une grande ouverture est faite en faveur des communautés locales, notamment en ce qui concerne les refuges locaux ("Article 97 : La gestion des refuges locaux relève de la compétence des collectivités territoriales décentralisées, qui bénéficient à cet effet de l'assistance des services techniques locaux chargés de la faune. Les collectivités décentralisées prennent toutes les mesures nécessaires en vue d'assurer la participation effective des représentants des communautés concernées à cette gestion. En particulier, elles peuvent mettre en place des structures ad hoc de gestion fondée sur le partenariat, et dont la composition est déterminée par les textes complémentaires et d'application.»), les zones villageoises d'intérêt cynégétique («Article 101: Les activités autorisées à l'intérieur des zones villageoises d'intérêt cynégétique sont déterminées par les communautés de base avec l'assistance des services techniques locaux chargés de la faune»), la répartition des revenus tirés de la faune ("Article 94 Les redevances et taxes collectées, dans le cadre de la valorisation des réserves de la faune, font l'objet d'une répartition entre le budget de l'Etat et celui des collectivités locales.»), etc.

Pour Ly (2001), les textes burkinabè sur les ressources fauniques, tout comme ceux des autres pays d'Afrique occidentale, repose sur 3 principes primordiaux. Il s'agit des principes de protection, de mise en valeur et de participation.

Outre le code forestier et la RAF qui traitent de la chasse et des ressources fauniques, certains textes récents sont importants à retenir :

- Le cahier des charges générales d'octobre 1995, régissant l'activité des concessionnaires de zones à vocation faunique au Burkina Faso.

- Le décret n ${ }^{\circ}$ 96-061/PRES/PM/MEE/MATS/MEFP/MCIA/MTT du 11 mars 1996, portant réglementation de l'exploitation de la faune au Burkina Faso.

- L'arrêté conjoint no 96-022/MEE/MICA/MEF du 23 décembre 1996, portant fixation des taxes, redevances et titres d'exploitation de la faune au Burkina Faso. 
- Le décret no 98-305/PRES/PM/MEE/MEF/MTT du 15 juillet 1998, portant réglementation des concessions de gestion de la faune et des activités de concessionnaire et de guide.

\subsection{Les textes sur les ressources pastorales}

Autant que le foncier, la situation des espaces pastoraux des terroirs est périlleuse. Généralement ces espaces de terroirs sont selon la terminologie de la loi d'orientation sur le pastoralisme, des espaces pastoraux ouverts, c'està-dire que leur destination principale est autre que pastorale, mais que les activités pastorales y sont autorisées, en concurrence ou non avec d'autres types d'activités rurales selon les périodes de l'année (CNSFMR 2007). Cette situation explique à elle seule le fait que l'utilisation de ces espaces soit source de nombreux conflits entre pasteurs et agriculteurs.

Etant de fait «Carrefour» de plusieurs activités rurales pour cause de lois toujours restées mitigées à leur égard, les espaces pastoraux interpellent le décideur, sur la question de leur appropriation, mais surtout sur les mesures à prendre pour organiser un accès équitable aux ressources naturelles au profit de l'ensemble des acteurs concernés.

En réponse à cette interpellation, le gouvernement a soumis à l'appréciation de l'assemblée nationale, une loi d'orientation sur le pastoralisme qui a été adoptée le 14 novembre 2002.

Si le code de l'environnement et, dans une moindre mesure, le code forestier font mention des espaces pastoraux, de leur création et de leur utilisation (tous les textes relatifs à la gestion et à l'aménagement de l'espace rural touche le pastoral), la loi d'orientation sur le pastoralisme constitue, à ce jour, la seule référence réglementaire en matière de pastoralisme.

La loi d'orientation sur le pastoralisme tente de sécuriser les espaces pastoraux dans leur diversité, ainsi que les pasteurs dans leur accès aux ressources naturelles (CNSFMR 2007). Son article premier donne l'objet de la loi ("La présente loi fixe les principes et les modalités d'un développement durable, paisible et intégré des activités pastorales, agropastorales et sylvopastorales»), tandis que l'article 3 donne la définition des ressources pastorales («...ressources végétales, hydriques, minérales exploitées dans le cadre de l'élevage pastoral, elles sont comprises soit dans les espaces affectés à pâture des animaux, soit dans les espaces ouverts à pâture des animaux»), des espaces pastoraux («espaces affectés et espaces ouverts à la pâture des animaux»), les espaces affectés à la pâture des animaux («les espaces dont 
la destination principale est l'exercice d'activités pastorales: les espaces pastoraux d'aménagement spécial, les espaces de terroir réservé à la pâture, les espaces de cultures fourragères destinés à la pâture directe des animaux»).

La section I du chapitre III du titre I de cette loi élucide les responsabilités de l'Etat et des CT. La loi responsabilise conjointement les CT et l'Etat, notamment en ses articles 5 et 6 :

"Article 5: L'Etat et les collectivités territoriales garantissent aux pasteurs le droit d'accès aux espaces pastoraux, le droit d'utilisation équitable des ressources naturelles et la mobilité des troupeaux.

L'Etat et les collectivités territoriales prennent également les mesures nécessaires à l'amélioration des performances productives et de qualité de l'élevage pastoral. En particulier, ils créent les conditions de sécurisation des activités pastorales et de préservation de la santé animale; ils encouragent et soutiennent les activités d'amélioration de l'alimentation des animaux.

Article 6: Dans le cadre de la politique d'aménagement du territoire, l'Etat et les collectivités territoriales veillentà l'identification, à la protection et à l'aménagement des espaces à vocation pastorale.

En particulier dans le cadre de l'aménagement des espaces périurbains, l'Etat et les collectivités territoriales réservent les espaces nécessaires à la poursuite et au développement des activités d'élevage.

L'Etat et les collectivités territoriales veillent également à la préservation et à la protection des espaces pastoraux traditionnels. En particulier, ils favorisent dans les régions à vocation pastorale, la préservation de la vocation pastorale des bas-fonds et des zones de bourgou.»

\subsection{Les textes sur les ressources halieutiques et piscicoles}

On entend par ressource halieutique tout organisme vivant dans l'eau et pouvant en être retiré (article $167 \mathrm{du}$ Code forestier). Le même code à son article 166 définit la pêche comme un acte qui consiste à la capture ou à l'extraction, par tout moyen autorisé et pour toute utilisation que ce soit, des ressources halieutiques contenues dans les eaux burkinabé, et l'aquaculture comme l'élevage d'organismes aquatiques, y compris notamment les poissons, les mollusques, les crustacés, les batraciens et les végétaux.

Le code forestier qui reste la grande référence en matière de gestion des ressources halieutiques et de la pisciculture consacre son Livre III à la pêche et de l'aquaculture. Les thèmes suivants y sont traités :

20 Textes et textes de loi sur la gestion des ressources naturelles au Burkina Faso 
- La réglementation générale et des principes de conservation des ressources

- La gestion et du développement des activités de la pêche

- La gestion et du développement des activités d'aquaculture

- La protection des eaux, de l'hygiène et de la qualité des produits halieutiques

En 1999, le Ministère en charge de la pêche en son temps (Ministère de l'Environnement et du Cadre de Vie) à travers un document du PNUD et du FEM (Bancé et al. 1999) s'est défini les options prioritaires suivantes :

- une meilleure organisation des pêcheurs;

- l'optimisation des captures par des normes et politiques adaptées ;

- la vulgarisation des technologies de transformation et de conservation des produits de la pêche.

Ces options collent bien avec celles définit par la PFN un an plus tôt (MECV 1998) :

- "La rationalisation de l'exploitation des ressources halieutiques à travers l'exploitation optimale du potentiel existant et les aménagements en vue de l'amélioration de la productivité et de l'augmentation de l'approvisionnement en poisson au regard du déficit constaté ;

- L'établissement de concessions de pêche au profit des populations riveraines en priorité ;

- Le développement de la pisciculture semi-intensive;

- La conservation des écosystèmes aquatiques naturels et les plans d'eau artificiels»

Toutes les interventions entreprises par le ministère en charge de la pêche (MET, puis MEE, jusqu'en juin 2002 et MAHRH depuis juin 2002) sont articulées autour de quatre approches: l'approche participative, l'approche programme, l'approche gestion des terroirs, l'approche par zone écologique (MECV 1998). Cette politique d'intervention a pour objectif principal de permettre aux populations riveraines des sites poissonneux (Kompienga, Bagré, Sourou) d'être les premiers bénéficiaires des revenus tirés de la pêche.

\subsection{Les textes sur les ressources minières et les carrières}

Le texte de référence pour la gestion des ressources minières et les carrières est sans conteste le code minier adopté en 2003. Ce code à son article 4 donne une définition de substances minérales en termes de «substances naturelles amorphes 
ou cristallines, solides, liquides ou gazeuses ainsi que les substances organiques fossilisées et les gîtes géothermiques», et donne la classification des gîtes naturels de substances minérales qui sont classés en mines et carrières :

- "Sont considérées comme carrières : les gîtes de matériaux de construction, d'empierrement et de viabilité, d'amendement pour la culture des terres ainsi que les substances servant à l'industrie céramique et autres substances analogues, à l'exception de phosphates, nitrates, sels alcalins et autres sels associés dans les mêmes gisements et les tourbières. Les carrières sont réputées ne pas être séparées du sol dont elles suivent le régime de propriété.

- Les gîtes naturels de substances minérales qui ne sont pas classés comme carrières sont considérés comme mines. Les mines constituent une propriété distincte de la propriété du sol.

- Les installations et facilités annexes sont soumises au même régime juridique que les gîtes naturels de substances auxquelles elles se rapportent. Sont considérées comme annexes les installations de toute nature nécessaires à l'exploitation.

- certains gîtes naturels de substances minérales peuvent toutefois être classés comme substances de carrières ou comme substances minières suivant l'usage auquel elles sont destinées dans les conditions prévues par la réglementation minière».

Ce code stipule que l'Etat est le seul propriétaire des ressources minérales. L'article 5 précise: "Les gîtes naturels de substances minérales contenus dans le sol et le sous-sol du Burkina Faso sont, de plein droit, propriété de l'Etat. L'Etat en assure la mise en valeur en faisant appel notamment à l'initiative privée conformément aux dispositions de la présente loi».

Toutefois, les collectivités locales sont consultées comme le prévoit l'article 45: "L'autorisation d'exploitation artisanale traditionnelle est accordée, sous réserve des droits antérieurs, par décision de l'Administration des mines, après consultation des autorités administratives compétentes et des collectivités locales concernées...». L'article 82 précise que les collectivités reçoivent même une part des taxes superficiaires versés par les titulaires de titre minier ou d'autorisation d'exploitation ("20\% du montant de la taxe superficiaire doit être versé à la collectivité où se trouve la superficie»).

La section 3 du chapitre 2 du titre 2 du code minier est consacrée à l'autorisation d'exploitation des carrières. Les populations locales sont là encore associées (consultées) à l'autorisation d'exploitation permanente que peut accorder l'administration à toute personne physique ou morale ayant présenté une demande conforme à la réglementation minière. 


\subsection{Autres documents de politiques prenant en compte les ressources naturelles}

Outre les textes mentionnés précédemment tout au long de ce document, faisons un arrêt sur d'autres textes dont la mise en œuvre a un impact sur la GRN. Il s'agit notamment des textes suivants :

\subsubsection{Lettre de Politique de Développement Rural Décentralisé}

Adoptée en juillet 2002 par le Gouvernement, la Lettre de Politique de Développement Rural Décentralisé (LPDRD) a pour objectif d'harmoniser les différentes approches pour la promotion du développement du monde rural autour des principes fondamentaux dont la responsabilisation totale des communautés de base impliquant une maîtrise d'ouvrage assurée par les bénéficiaires

\subsubsection{Cadre Stratégique de Lutte contre la Pauvreté}

Le Burkina Faso, à la faveur de son éligibilité à l'initiative d'allégement de la dette des pays pauvres très endettés (PPTE), a adopté un cadre stratégique de lutte contre la pauvreté (CSLP) en 2000, qui tire ses fondements de la Lettre d'Intention de Politique de Développement Humain Durable (LIPDHD) de 1995.

En 2003, un processus de révision de ce cadre stratégique a réactualisé la stratégie globale du gouvernement pour la réduction de la pauvreté au Burkina Faso en identifiant les grands principes (CSLP-2) dont la gestion durable des ressources naturelles.

Le CSLP prend en compte les ressources naturelles à travers trois points : la gestion des ressources en terre, la gestion des ressources en eau, la gestion des ressources forestières, fauniques et halieutiques. En effet, seule la gestion durable et bénéfique des ressources agro-sylvopastorales conditionne largement la réussite du CSLP.

\subsubsection{Stratégie de Développement Rural à l'horizon 2015}

Elaborée en cohérence avec le Cadre Stratégique de Lutte contre la Pauvreté (CSLP), la Stratégie de Développement Rural (SDR) a été adoptée par le Gouvernement le 30 décembre 2003.

La SDR à l'horizon 2015 a pour objectif global d'assurer une croissance soutenue du secteur rural en vue de contribuer à la lutte contre la pauvreté, au renforcement de la sécurité alimentaire et à la promotion d'un développement 
durable. Parmi les objectifs spécifiques de la sont SDR, deux sont rattachés à la GRN (MECV 2007-a) :

- accroître les productions agricoles, pastorales, forestières, fauniques et halieutiques grâce à l'amélioration de la productivité ;

- assurer une gestion durable des ressources naturelles ;

Cadre de référence de l'ensemble des interventions publiques en faveur du développement rural, la SDR, s'est définit des axes stratégiques dont trois sont en relation avec la GRN (MECV 2007-a) :

- accroître, diversifier et intensifier les productions agricoles, pastorales, forestières, halieutiques et fauniques ;

- améliorer l'approvisionnement en eau potable ;

- assurer une gestion durable des ressources naturelles ;

\subsubsection{Stratégie nationale de gestion des feux en milieu rural}

Pour permettre d'atténuer les impacts négatifs des feux tout en valorisant leurs impacts positifs dans un contexte de développement durable, le Burkina Faso s'est doté d'une stratégie nationale et d'un plan d'action pour la gestion des feux en milieu rural en 2007. Les interventions définies par ladite stratégie devront être réalisées à travers ses principaux axes d'orientations qui sont (MECV 2007-a) :

- assurer une maîtrise foncière sur les espaces des terroirs ;

- faire de la gestion des feux en milieu rural, une responsabilité locale ;

- renforcer les compétences et les capacités des Collectivités Territoriales, des institutions villageoises et des organisations de la société civile en matière de gestion des feux ;

- renforcer le développement des capacités techniques de prestataires privés ;

- renforcer les capacités des services techniques.

\subsubsection{Le Programme d'action national d'adaptation à la variabilité et aux changements climatiques}

En fin 2007, le gouvernement du Burkina Faso adopte un programme d'action national d'adaptation à la variabilité et aux changements climatiques (PANA). 
Le contenu de ce document expose le programme d'adaptation du pays. Il rappelle les caractéristiques essentielles de celui-ci, les principales pressions exercées sur l'environnement et la manière dont les changements climatiques perturbent les processus biophysiques. Il décrit également le cadre du programme d'adaptation, dresse la liste des besoins essentiels en matière d'adaptation, précise les critères de sélection des actions prioritaires d'adaptation, propose une liste hiérarchisée de douze actions prioritaires et en établit des fiches de projet pour leur mise en œuvre. Il se termine sur un rappel du processus adopté pour l'élaboration du programme d'adaptation du pays aux changements et à la variabilité climatiques (MECV 2007-b).

\subsubsection{La politique nationale d'aménagement du territoire}

\section{Le décret $\mathrm{n}^{\circ}$ 2006-362/PRES/PM/MEDEV/MATD/MFB/ MAHRH/} MID/MECV du 20 juillet 2006 portant adoption de la politique nationale d'aménagement du territoire, donne le cadre réglementaire de l'aménagement du territoire conformément à la RAF et au CGCT. Le paragraphe introductif de ce décret montre l'évolution de la notion d'aménagement du territoire au Burkina Faso. "Si la pratique de l'aménagement du territoire est ancienne au Burkina Faso, son introduction dans le système national de planification (SNP) est relativement récente. En effet, c'est la planification régionale qui a été d'abord prise en charge par la création des services départementaux du plan et de la statistique (SDPS). Par cette mesure, il s'agissait de prendre en compte les réalités régionales dans les analyses pour mieux localiser les projets d'investissement, apporter une réponse aux problèmes de l'exode rural et ses conséquences, à l'aggravation des disparités régionales en matière d'équipements et d'infrastructures, à la répartition optimale des activités et des populations sur le territoire. Cette action de mise en place des structures de planification régionale fut complétée en 1979 par la création de la Direction de l'aménagement du territoire et plus tard par d'autres services départementaux du plan et de la statistique, traduisant la volonté du gouvernement d'utiliser l'aménagement du territoire pour la conduite du développement..

Cette politique consacre l'élaboration d'un certain nombre de document de planification ou la mise en œuvre d'autres préalablement élaborés. On peut citer entre autres :

- le schéma directeur de cartographie du territoire ;

- le programme d'action national pour l'environnement ;

- le programme d'action national de lutte contre la désertification ;

- le plan d'action de gestion intégrée des ressources en eau ; 
- le plan d'action et programme d'investissement du secteur d'élevage

- la stratégie nationale et le plan d'action en matière de biodiversité

- la stratégie nationale de mise en œuvre de la convention sur les changements climatiques

- la lettre de politique de développement rural décentralisée, etc.

- schémas régionaux et provinciaux d'aménagement du territoire.

Outre le développement économique et l'intégration sociale, la gestion durable du milieu naturel constitue l'une des orientations majeures de cette politique. Cela revient donc à la recherche permanente du compromis entre d'une part, la nécessité d'exploiter les ressources de l'environnement pour assurer une existence décente à l'homme d'aujourd'hui et d'autre part les impératifs de préserver les bases nécessaires à la survie de l'homme d'aujourd'hui et de celui de demain. A cette troisième orientation, qui a trait à la préservation de l'environnement et à la valorisation des ressources naturelles, sont rattachés les objectifs suivants :

- la sécurisation foncière,

- la réhabilitation et la restauration des ressources naturelles dégradées et/ou fortement compromises ;

- la mise en valeur durable du potentiel environnemental ;

- l'amélioration du cadre de vie urbain et rural ;

- la protection à grande échelle des terres ;

- la gestion durable des ressources agricoles et pastorales,

- la mise en adéquation entre le système économique et les potentialités naturelles. 


\section{III}

Les conventions locales pour la GRN

\subsection{La définition d'une convention locale}

La convention est un accord volontaire entre deux ou plusieurs parties ou personnes. Elle définit les relations entre les parties, les conditions d'utilisation, d'exploitation ou de gestion de ce qui fait l'objet de l'accord. Dans le cadre des conventions locales sur la GRN, il s'agit des clauses d'utilisation et de conservation de ces ressources et des relations qu'entretiennent les différentes parties prenantes, notamment les populations locales environnantes.

Dans le projet d'arrêté conjoint (MECV, MAHRH et MATD) portant contenu, démarche élaboration et validation des conventions locales de gestion des ressources forestières la définition suivante est donnée : «Le code local ou convention locale de gestion des ressources naturelles est un ensemble de normes et règles déterminant l'utilisation des ressources naturelles dans un terroir donné ainsi que les sanctions décidées en cas d'abus. C'est un chaînon du Plan de Gestion des Terroirs. En d'autres termes, les conventions ou règles internes de gestion des ressources naturelles (RIGRN) sont des règles consensuelles, des modalités arrêtées par consensus entre d'une part les populations et d'autre part les partenaires techniques et les autorités politico-administratives et décentralisées» (article 1).

\subsection{La place des conventions locales}

Les textes réglementaires (loi, décret, arrêté, politique relative à un secteur, programme, plan national, etc.) règlent généralement des cas généraux et restent incapables à prendre en compte toutes les spécificités de toutes les régions couvertes par ces textes. Ces textes reconnaissent et encouragent fréquemment d'ailleurs l'élaboration de conventions locales pour la GRN. 
Ces conventions qui sont soumises à la législation nationale ne peuvent ni concurrencer, ni remplacer les textes en vigueur. Elles sont faites juste pour résoudre des questions locales spécifiques. Leur rôle principal est de limiter les conflits autour de l'exploitation de ces ressources.

\subsection{Le texte sur les conventions locales}

Outre le CGCT qui ouvre la porte de négociation de conventions aux collectivités locales, la référence valide en matière de conventions locales sur la GRN au Burkina constitue le projet d'arrêté conjoint portant contenu, démarche élaboration et validation des conventions locales de gestion des ressources forestières, cité dans le «Plan d'action de mise en ouvre des reformes institutionnelles et juridiques pour la décentralisation dans le secteur forestier» publié par le MECV en 1998. Il faut cependant noter que plusieurs projets, programmes et organismes de développement (la GTZ, la SNV, la coopération danoise, etc.) ont encouragé et soutenu des organisations rurales pour l'élaboration et la mise en œuvre de conventions locales. Mais cet élan n'a pas été soutenu par un cadre de référence ou un texte officiel d'orientation.

\subsection{Les signataires des conventions locales}

Il va de soit que les signataires d'une convention locale soient les parties prenantes. Dans le projet d'arrêté relatif aux conventions locales, «les parties à l'élaboration d'une convention locale sont constituées par:

- les populations locales organisées au sein de structures de gestion;

- les partenaires techniques (ONG, projets, services techniques déconcentrés de l'Etat);

- les autorités politico-administratives (préfet, haut-commissaire);

- les autorités décentralisées (les maires et les conseils municipaux)» (article 3).

Le conseil communal peut procéder à la prise d'arrêtés portant approbation des conventions locales de GRN. Le projet d'arrêté va plus loin en précisant à l'article 6 que "Pour qu'une convention locale soit valide, il faut qu'elle soit reconnue par le premier responsable de la collectivité territoriale (maire de la commune rurale) du ressort territorial de l'implantation de la ressource. Cette reconnaissance doit être matérialisée par la signature de l'autorité communale sur la convention locale».

Le comité villageois de développement (CVD) peut proposer au conseil communal l'adoption de conventions locales de gestion de certaines ressources partagées comme par exemple les zones sylvopastorales, dans le but d'en assurer l'optimisation socio-économique 


\subsection{Les phases d'élaboration d'une convention locale}

Selon le projet d'arrêté sur les conventions locales sur la gestion des ressources forestières, les différentes phases d'élaboration d'une convention locale sont :

- L'initiative de l'élaboration: elle appartient aux populations locales organisées au sein des structures de gestion (associations locales, CVD), appuyées par les services techniques et partenaires au développement qui les accompagnent dans l'élaboration proprement dite. Cette initiative peut aussi provenir de l'autorité communale (conseil municipal de la commune rurale) pour protéger spécialement une ressource bien définie.

- La phase d'information/sensibilisation des populations locales et des structures déconcentrées de l'Etat: cette phase doit consister en des rencontres et réunions d'information des populations locales afin de dégager de manière précise le domaine et le champ d'application de la convention. Les populations sont généralement assistées de consultants et de techniciens des services techniques qui sont impliqués au titre de conseillers pour faciliter la recherche de consensus et assurer la conformité des textes à la juridiction légale et dans l'explication des thèmes dans leur formulation. Une fois la formulation faite, l'avant projet de convention est discuté avec les techniciens des structures déconcentrées de l'Etat et les autorités politico-administratives qui apportent leurs amendements.

- La phase d'adoption : elle consiste en une grande réunion de toutes les parties prenantes autour de l'autorité politico-administrative du ressort de la convention pour l'adoption définitive de la convention locale.

- La validation de la convention locale : comme signalé plus haut, une convention locale n'est valide que si elle est reconnue par le premier responsable de la collectivité territoriale (maire de la commune rurale).

Une fois la convention locale authentifiée et validée par les autorités politicoadministratives et communales du ressort du projet, elle est mise en œuvre et n'est applicable que dans la zone du ressort territorial couvert par le projet (article 7 du projet d'arrêté). 


\section{Conclusion}

Les RN sont une des principales sources de moyens de subsistance pour les populations rurales au Burkina Faso: alimentation, revenus, soins, etc. une gestion durable de ces ressources est indispensable pour la survie de la génération actuelle, et pour garantir aux générations futures un environnement équilibré.

La réussite de ce pari de gestion durable passe par le respect de l'environnement. En vu d'y parvenir, plusieurs textes législatifs et réglementaires ont été adoptés.

Mais dans leur application, certaines lacunes subsistent :

- Certains textes tels que la RAF semblent en porte-à-faux avec la réalité du terrain. En effet face à un régime coutumier qui confere le droit de propriété de la terre à une organisation coutumière et son acquisition par l'héritage et le don, la loi dite moderne proclame l'Etat comme seul propriétaire de la terre. La reconnaissance (ou la prise en compte) du droit coutumier est indispensable pour une gestion non conflictuelle des RN d'une manière générale et du foncier de façon particulière en milieu rural ;

- Les textes sont méconnus des populations locales, faute de traduction et de vulgarisation ;

- Les conventions locales qui sont l'outil approprié aux spécificités locales et qui s'intègrent parfaitement au tissu institutionnel, législatif et réglementaire de la décentralisation devraient être mises à la disposition des populations locales qui doivent s'en approprier.

Cependant, malgré les imperfections qu'on peut relever, les textes ont le mérite de proposer des pistes, des règles et des principes de gestion durable des RN.

Toutefois, le gros problème reste le transfert effectif du domaine de GRN aux CT pour une véritable gestion décentralisée des $\mathrm{RN}$ au profit des populations environnantes. 


\section{Références}

Ibrahima Ly 2001. Tendances d'évolution du droit de la faune et des aires protégées en Afrique occidentale. Etude juridique de la FAO en ligne \# 13. Janvier 2001

Jesse Ribot. 1999. "Decentralization, Participation and Accountability in Sahelian Forestry: Legal Instruments of Political-Administrative Control," Africa, Vol. 69, No. 1, January 1999.

Jesse Ribot. 2001. Science, use rights and exclusion: a history of forestry in francophone West Africa). WRI. Washington, USA. 19 pages

Le Petit Larousse illustré. 2000, page 886 (définition de ressource). Larousse. Paris, France. Page 886.

Matthias Banzhaf, Boureima Drabo, Hermann Grell. 2000. Du conflit au consensus : Les pasteurs et agro-pasteurs de Kishi Beiga (Burkina Faso) optent pour la cogestion de leurs ressources naturelles. Securing the commons No3. Août 2000

Ministère del'Agriculture, de l'Hydraulique et des Ressources Halieutiques. 2007. Politique nationale de sécurisation foncière en milieu rural. MAHRH. Ouagadougou, Burkina Faso. 49 pages

Ministère de l'Environnement et du Tourisme. 1991. Le Plan d'action national pour l'environnement (PANE), Tome $1,2^{\text {e }}$ édition. MET. Ouagadougou, Burkina Faso. 183 pages

Ministère de l'Environnement et du Cadre de Vie. 1998. Politique Forestière Nationale. MECV. Ouagadougou, Burkina Faso. 24 pages

Ministère de l'Environnement et du Cadre de Vie. 2006. Plan d'action de mise en ouvre des reformes institutionnelles et juridique pour la décentralisation dans le secteur forestier

Ministère de l'Environnement et du Cadre de Vie. 2007 (a). Programme National de Gestion des Ressources Forestières 2006 - 2015. MECV. Ouagadougou, Burkina Faso. 87 pages 
Ministère de l'Environnement et du Cadre de Vie. 2007 (b). Programme d'action national d'adaptation à la variabilité et aux changements climatiques (PANA) du Burkina Faso. MECV. Ouagadougou, Burkina Faso. 84 pages

Soumayila BANCE et al. 1999. Stratégie nationale et plan d'action du Burkina Faso en matière de diversité biologique. PNUD et FEM 


\section{Annexes}

1. La liste de lois relatives à la GRN votées par l'AN de 1992 à 2007

2. Liste des principaux textes sur la GRN

3. Le code de l'environnement

4. Le code forestier

5. La loi d'orientation relative à la gestion de l'eau

6. La loi d'orientation relative au pastoralisme

7. Le Code Général des Collectivités Territoriales (CGCT) 


\section{Annexe I}

\section{LISTE DE LOIS RELATIVES A LA GRN VOTEES PAR L'AN DE 1992 A 2007}

\begin{tabular}{|c|c|c|}
\hline $\mathbf{N}^{\circ}$ de loi & Intitulé de la loi & $\begin{array}{c}\text { Date } \\
\text { adoption }\end{array}$ \\
\hline 031-2003/AN & Loi portant code minier au Burkina Faso. & $08-05-03$ \\
\hline 02-2001/AN & Loi d'orientation relative à la gestion de l'eau & 08-02-01 \\
\hline 23/97/II/AN & Loi portant Code Minier au Burkina Faso & $22-10-97$ \\
\hline 06/97/ADP & Loi portant code forestier au Burkina Faso & $31-01-97$ \\
\hline 05/97/ADP & Loi portant code de l'environnement au Burkina Faso & $30-01-97$ \\
\hline 20/96/ADP & $\begin{array}{l}\text { Loi portant institution d'une taxe de jouissance pour } \\
\text { l'occupation et la jouissance des terres du domaine } \\
\text { foncier national appartenant à l'Etat }\end{array}$ & 10-07-96 \\
\hline 06/95/ADP & $\begin{array}{l}\text { Loi portant modification de la loi } n^{\circ} 014 / 93 / \text { ADP portant } \\
\text { code des investissements miniers }\end{array}$ & $26-04-95$ \\
\hline 02/94/ADP & Loi portant code de l'environnement au Burkina Faso & $11-03-94$ \\
\hline 14/93/ADP & Loi portant code des investissements miniers & $19-05-93$ \\
\hline
\end{tabular}

34 Textes et textes de loi sur la gestion des ressources naturelles au Burkina Faso 


\section{Annexe II}

\section{LISTE DES PRINCIPAUX TEXTES RELATIFS A LA GRN}

1. La Lettre de Politique de Développement Rural Décentralisé (LPDRD)

2. La lettre de politique de développement rural décentralisée

3. La loi d'orientation relative au pastoralisme

4. La loi d'orientation sur la gestion de l'eau

5. La Politique Forestière Nationale (PFN)

6. La politique nationale d'aménagement du territoire

7. la Politique nationale de développement durable de l'agriculture irriguée et le Plan d'action y relatif pour l'horizon 2015

8. La Politique nationale de sécurisation foncière en milieu rural (PNSFMR)

9. La réorganisation agraire et foncière (RAF)

10. La Stratégie nationale de gestion des feux en milieu rural

11. La Stratégie nationale de l'assainissement

12. La stratégie nationale de mise en œuvre de la convention sur les changements climatiques

13. La stratégie nationale et le plan d'action en matière de biodiversité

14. Le Cadre Stratégique de Lutte contre la Pauvreté (CSLP)

15. Le code de l'environnement

16. Le code forestier

17. Le code général des collectivités territoriales (CGCT)

18. Le code minier

19. Le Plan d'Action de Gestion Intégrée des Ressources en Eau (PAGIRE)

20. Le plan d'action de mise en ouvre des reformes institutionnelles et juridiques pour la décentralisation dans le secteur forestier

21. Le plan d'action et programme d'investissement du secteur d'élevage

22. Le Plan d'Action National pour l'Environnement (PANE)

23. Le Plan d'Action National de Lutte Contre la Désertification (PAN/ LCD)

24. Le Programme d'action National d'Adaptation à la variabilité et aux changements climatiques (PANA)

25. Le Programme National d'Aménagement des Forêts (PNAF)

26. Le Programme National de Foresterie Villageoise (PNFV)

27. Les schémas régionaux et provinciaux d'aménagement du territoire

28. Le programme national de gestion des ressources forestières (PNGRF) 


\section{Annexe III}

\section{LOI N 005/97/ADP PORTANT CODE DE L'ENVIRONNEMENT AU BURKINA FASO}

\section{L'ASSEMBLEE DES DÉPUTÉS DU PEUPLE}

$\mathrm{Vu}$ la Constitution;

$\mathrm{Vu} \quad$ la Résolution $\mathrm{n}^{\circ}$ 001/92/ADP du 17 juin 1992, portant validation du mandat des Députés ;

A délibéré en séance du 30 janvier 1997

et adopté la loi dont la teneur suit :

\section{PREAMBULE}

Pays sahélien et enclavé dont l'économie repose essentiellement sur l'agriculture et l'élevage, le Burkina Faso connaît une dégradation continue de son écosystème.

Cette situation, accentuée par les sécheresses persistantes, a amené le pays à prendre conscience de la nécessité d'intégrer l'environnement et le développement à travers les options fondamentales suivantes qui impliquent outre la responsabilité de l'Etat, celle des collectivités locales décentralisées et des individus à tous les niveaux :

- la prise en compte del'interdépendance entrel'environnement, le développement socioéconomique et la qualité de vie dans tous programmes et projets de développement ;

- la ratification des accords internationaux en matière de préservation de l'environnement;

- la préservation des générations futures des calamités naturelles et artificielles liées à la dégradation de l'environnement.

Aussi, la présente loi dont le préambule fait partie intégrante a été élaborée pour servir de source d'inspiration de tous les textes qui seront pris pour répondre aux aspirations profondes de notre peuple en matière de préservation de l'environnement.

\section{TITRE I : DES DISPOSITIONS GENERALES}

\section{CHAPITRE I : DE L'OBJET ET DU CHAMP D'APPLICATION}

ARTICLE 1 : Le présent Code vise à établir les principes fondamentaux destinés à préserver l'environnement et à améliorer le cadre de vie au Burkina Faso.

ARTICLE 2 : Les principes fondamentaux de préservation de l'environnement visés à l'article 1 ci-dessus sont :

- la lutte contre la désertification ;

- l'assainissement et l'amélioration du cadre de vie des populations urbaines et rurales ;

- la mise en œuvre des Accords Internationaux ratifiés par le Burkina Faso en matière de préservation de l'Environnement ;

- la prévention et la gestion des catastrophes. 
ARTICLE 3 : Les dispositions du présent Code s'appliquent sans préjudice de celles relatives à l'Environnement et relevant des domaines spécifiques régis par la loi portant Réorganisation Agraire et Foncière (RAF), le Code de la Santé Publique, la loi sur le contrôle des pesticides et le Code Pénal.

ARTICLE 4 : La prévention et la gestion des catastrophes naturelles et artificielles font l'objet d'un décret pris en Conseil des Ministres, sur proposition conjointe des Ministres chargés de l'Action Sociale, de l'Administration du Territoire et de l'Environnement, en concertation avec toutes les parties concernées.

\section{CHAPITRE II : DE L'EMPLOI DES TERMES}

ARTICLE 5 : Au sens du présent Code,

1) LEnvironnement est l'ensemble des éléments physiques, chimiques et biolo-giques naturels ou artificiels et des facteurs économiques, sociaux, politiques et culturels, qui ont un effet sur le processus de maintien de la vie, la transformation et le développement du milieu, les ressources naturelles ou non et les activités humaines.

2) La Dette Ecologique est une redevance perçue au titre de l'exploitation et/ou de l'utilisation à des fins lucratives, des ressources naturelles ou de l'environnement.

3) L'Education Environnementale est l'ensemble des actions de sensibilisation, de formation et d'information visant à responsabiliser les populations sur la nécessité absolue de promouvoir un environnement sain.

4) L'Etude d'Impact sur l'Environnement (E.I.E.) est une étude à caractère analytique et prospectif aux fins de l'identification et de l'évaluation des incidences d'un projet sur l'environnement.

5) La Notice d'Impact sur l'Environnement (N.I.E.) est une E.I.E. simplifiée. Toutefois, elle doit répondre aux mêmes préoccupations que l'E.I.E. et comporter des indications sérieuses.

L'Etude et la Notice d'Impact sur l'Environnement permettent de cerner la différence entre l'environnement futur modifié tel qu'il résultera de l'exécution d'une activité, et l'environnement futur tel qu'il aurait évolué normalement sans la réalisation de ladite activité.

6) Les établissements dangereux, insalubres ou incommodes sont ceux présentant des dangers ou des inconvénients, soit pour la commodité du voisinage, soit pour la santé et la sécurité publique, soit pour l'agriculture, le cadre de vie, la conservation des sites, espaces, monuments et la diversité biologique.

7) Les déchets urbains sont des détritus solides, liquides ou gazeux en provenance des maisons d'habitation et assimilés, des immeubles administratifs, des salles de spectacles, de restauration et de tout autre établissement recevant du public.

Sont assimilées à des maisons d'habitation les casernes et les écoles.

Sont compris dans la dénomination déchets urbains :

- les ordures ménagères, les cendres, les débris de verre ou de vaisselle, les emballages plastiques, les feuilles, les balayures et les résidus de toute sorte, déposés dans des récipients individuels ou collectifs placés devant les maisons ou sur la voie publique, les eaux usées domestiques, les excréta ;

- les déchets non industriels, les déchets assimilés à des déchets urbains des établissements industriels, les déchets des établissements commerciaux, bureaux, cours et jardins privés, déposés dans des récipients, dans les mêmes conditions que les ordures ménagères ; 
- les crottins, lisiers, fumiers, feuilles mortes, boues et d'une façon générale, tous les produits provenant du nettoiement des voies publiques, voies privées abandonnées au balayage, jardins publics, parcs, cimetières et leurs dépendances, rassemblés en vue de leur évacuation ;

- les produits du nettoiement et détritus des halles, foires, marchés, lieux des fêtes publiques, lieux d'attache des bêtes de somme ou de trait, rassemblés en vue de leur évacuation ;

- les résidus en provenance des écoles, casernes, hospices, prisons et tout bâtiment public, groupés sur des emplacements déterminés, dans des récipients appropriés, les résidus non toxiques et non dangereux des formations sanitaires et assimilées ;

- le cas échéant, tous objets abandonnés sur les lieux, places et voies publiques ainsi que les cadavres d'animaux.

8) Est déchet industriel ou assimilé, tout résidu qu'il soit liquide, solide ou gazeux, issu d'un processus de fabrication, de transformation ou d'utilisation d'une matière ou d'un produit.

Sont ainsi dénommés :

- les déchets des industries chimiques, combustibles ou incombustibles, les produits phytosanitaires obsolètes, les boues d'épuration des eaux traitées, les boues industrielles, les huiles usagées, les émanations gazeuses, les eaux usées industrielles et artisanales, les ferrailles et les épaves de véhicules de tout genre ;

- les déchets toxiques ou pathogènes des formations sanitaires et assimilées.

9) Est dangereux, tout déchet présentant des risques graves pour la santé et la sécurité publique et pour l'environnement.

Les déchets en provenance de l'étranger sont présumés dangereux.

10) Les pesticides et assimilés sont :

- les produits de protection et d'amélioration des végétaux et des récoltes ;

- les produits de désinfection des formations sanitaires et assimilés, des établissements publics ou privés et des ménages ;

- les adjuvants vendus seuls ou en mélange, destinés à améliorer les conditions d'utilisation des produits ci-dessus définis ;

- Les produits antiparasitaires à usage vétérinaire qui sont des produits chimiques organiques ou minéraux destinés au diagnostic, à la prévention et au traitement des maladies parasitaires des animaux.

11) Les matières fertilisantes sont les engrais organiques et minéraux et des amendements dont l'emploi contribue d'une manière générale à améliorer les propriétés physiques, chimiques et biologiques du sol et à assurer la nutrition des végétaux.

12) Les pollutions atmosphériques se caractérisent par :

- la présence dans l'air ambiant de substances ou particules qui, de par leurs aspects, leurs concentrations, leurs odeurs ou leurs effets physiologiques, portent préjudice à la santé et à la sécurité publique ou à l'environnement;

- la présence dans l'atmosphère de substances contribuant entre autre à l'effet de serre et à la réduction de la couche d'ozone.

13) La pollution ou l'acte de pollution des eaux et des sols consistent en toutes modifications des caractéristiques physiques, chimiques et biologiques des eaux et des sols compromettant ainsi les usages qui en sont faits ou qui pourraient en être faits.

Les types de pollutions possibles des eaux et des sols sont : 
- la pollution physico-chimique;

- la pollution biologique;

- la pollution radioactive.

14) L'acte d'assainissement consiste en la gestion :

- des déchets solides, liquides ou gazeux provenant des ménages, des établissements publics ou privés, des industries, de l'artisanat et des exploitations agricoles ;

- des eaux de pluie ou de ruissellement ;

- des végétaux et animaux envahissants.

15) Les nuisances diverses sont entre autres :

- les bruits et émissions sonores ;

- les odeurs incommodantes ;

- les émissions lumineuses vives ;

- les fumées de cigarettes, cigares et pipes, lorsque ceux-ci sont de nature à nui re au repos, à la tranquillité, à la santé, à la sécurité publique ou à d'autres égards.

16) Les aménagements paysagers sont constitués par l'ensemble des opérations d'embellissement visant à améliorer le cadre de vie.

CHAPITRE III : DU CADRE INSTITUTIONNEL

ARTICLE 6 : Le Ministère chargé de l'environnement est le garant de la coordination institutionnelle de la qualité de l'environnement au Burkina Faso.

\section{TITRE II - DE LA PRESERVATION DE LENVIRONNEMENT ET DE L'AMELIORATION DU CADRE DE VIE}

\section{CHAPITRE I - DES OUTILS DE PRESERVATION DE L'ENVIRONNEMENT SECTION 1 - DU CADRE DE CONCERTATION, D'ORIENTATION, DE SUIVI ET D'EVALUATION}

ARTICLE 7 : Il est institué un cadre de concertation, d'orientation, de suivi et d'évaluation, en vue de l'intégration des principes fondamentaux de préservation de l'environnement dans le processus de développement social, économique et culturel du Burkina Faso.

ARTICLE 8 : La création, l'organisation, les attributions et le fonctionnement du cadre visé à l'article 7 ci-dessus, font l'objet d'un décret pris en Conseil des Ministres sur proposition du Ministre chargé de l'environnement.

ARTICLE 9 : Sont soumis au suivi ou à l'appréciation du cadre visé à l'article 7 ci-dessus, tous les projets de développement ayant un impact important sur l'environnement.

Un décret pris en Conseil des Ministres sur proposition du Ministre chargé de l'environnement, établit la liste des différents types de plans, programmes, projets et activités soumis aux prescriptions de l'alinéa 1 du présent article.

SECTION 2 - DU CONTROLE DE LA QUALITE

ARTICLE 10 : Il est institué au Burkina Faso un contrôle de la qualité de l'environnement, des produits et des denrées.

Un décret pris en Conseil des Ministres sur proposition conjointe du Ministre chargé de l'environnement et des Ministres concernés, fixe les normes de qualité de l'environnement, des produits et denrées, applicables au Burkina Faso. 


\section{SECTION 3 - DU FONDS D'INTERVENTION POUR L'ENVIRONNEMENT(F.I.E)}

ARTICLE 11 : Il est institué un Fonds d'Intervention pour l'Environnement (F.I.E).

Ce Fonds est exclusivement réservé au financement des opérations de restauration de l'environnement, de lutte contre les pollutions et nuisances, et à toute action écologique conformément aux principes fondamentaux de préservation de l'environnement.

ARTICLE 12 : L'organisation, les modalités de fonctionnement et d'alimentation ainsi que les conditions d'utilisation du Fonds d'Intervention pour l'Environnement sont déterminées par décret pris en Conseil des Ministres sur proposition conjointe des Ministres chargés de l'Environnement et des Finances.

ARTICLE 13 : Il est institué une Dette Ecologique (D.E) au Burkina Faso au titre de l'exploitation et de l'utilisation à des fins lucratives des ressources naturelles ou de l'environnement, sans préjudice de la réparation des dommages causés à l'environnement.

ARTICLE 14 : La Dette Ecologique est due sous forme de redevance, dans les conditions fixées par décret pris en Conseil des Ministres, sur proposition des Ministres concernés.

Elle participe à l'alimentation du Fonds d'Intervention pour l'Environnement (F.I.E) .

\section{SECTION 4 - DE L'EDUCATION ENVIRONNEMENTALE}

ARTICLE 15 : Il est institué au Burkina Faso, une Education Environnementale dans tous les plans, programmes et projets de développement ainsi que dans les ordres d'enseignement pour faciliter la prise en compte des principes fondamentaux de préservation de l'environnement dans le développement socio-économique du pays.

ARTICLE 16 : Les Ministères chargés de l'Education, de la Communication, de l'Environnement et tout autre Ministère concerné, élaborent les modules de formation en environnement, déterminent le public cible ainsi que les voies et moyens de diffusion des messages.

\section{SECTION 5 - DES ETUDES ET DES NOTICES D'IMPACT SUR L'ENVIRONNEMENT}

ARTICLE 17 : Les activités susceptibles d'avoir des incidences significatives sur l'environnement sont soumises à l'avis préalable du Ministre chargé de l'Environnement. L'avis est établi sur la base d'une Etude d'Impact sur l'Environnement (E.I.E) ou d'une Notice d'Impact sur l'Environnement (N.I.E) soumise à l'examen du cadre visé à l'article 7 ci-dessus.

ARTICLE 18 : L'Etude et la Notice d'Impact sur l'Environnement, s'inscrivent à l'intérieur d'un processus décisionnel. De ce fait elles contribuent à établir la faisabilité des projets au même titre que les études techniques, économiques et financières.

ARTICLE 19 : L'Etude d'Impact sur l'Environnement doit être complétée par une enquête publique dont le but est de recueillir les avis et les contre propositions des parties concernées par rapport à l'Etude d'Impact sur l'Environnement qui est présentée.

Les conditions d'exécution de l'enquête publique sont fixées par décret pris en Conseil des Ministres sur proposition du Ministre chargé de l'Environnement.

40 Textes et textes de loi sur la gestion des ressources naturelles au Burkina Faso 
ARTICLE 20 : Un décret pris en Conseil des Ministres sur proposition du Ministre chargé de l'Environnement établit et révise la liste des travaux, ouvrages, aménagements et activités, ainsi que les documents de planification assujettis à l'Etude ou à la Notice d'Impact sur l'Environnement.

Ce décret précise les conditions dans lesquelles l'Etude ou la Notice d'Impact sur l'Environnement doit être rendue publique.

ARTICLE 21 : Tout promoteur peut avoir recours à une expertise de son choix pour effectuer l'Etude ou la Notice d'Impact sur l'Environnement. Les résultats de ces études font l'objet d'un rapport.

ARTICLE 22 : Les frais inhérents à la réalisation de l'Etude ou de la Notice d'Impact sur l'Environnement sont entièrement à la charge du promoteur.

ARTICLE 23 : Un décret pris en Conseil des Ministres, sur proposition du Ministre chargé de l'Environnement, détermine le contenu de l'Etude et de la Notice d'Impact sur l'Environnement.

ARTICLE 24 : La gestion des problèmes environnementaux à caractère transfrontalier se fait en concertation avec le(s) Etat(s) concerné(s) dans le respect des normes internationales en la matière.

\section{CHAPITRE II : DES MESURES DE PRESERVATION DE L'ENVIRONNEMENT SECTION 1 - DES MESURES SUR LES ETABLISSEMENTS DANGEREUX, INSALUBRES ET INCOMMODES}

ARTICLE 25 : Les établissements dangereux, insalubres et incommodes sont répartis en trois classes :

- la première classe comprend les établissements qui, de par leur nature, doivent être obligatoirement éloignés des habitations ;

- la deuxième classe comprend les établissements dont l'éloignement des habitations n'est pas rigoureusement nécessaire, mais dont l'exploitation ne peut être autorisée qu'à la condition que des mesures soient prises pour prévenir les dangers ou les incommodités ;

- la troisième classe comprend les établissements qui, bien que ne présentant pas d'inconvénients graves, ni pour le voisinage, ni pour la santé et la sécurité publique, sont cependant soumis à des prescriptions générales édictées pour tous les établissements similaires.

ARTICLE 26: Un décret pris en Conseil des Ministres sur proposition du Ministère chargé de l'Environnement classe les établissements installés au Burkina Faso conformément aux dispositions de l'article 25 ci-dessus.

Cette classification doit s'adapter à celles des filières déjà sélectionnées par les Ministères chargés de l'Industrie et du Commerce.

ARTICLE 27 : Les conditions d'ouverture et de fonctionnement des établissements visés à l'article 26 ci-dessus sont déterminées par décret pris en Conseil des Ministres sur proposition conjointe du Ministre chargé du secteur d'activité concerné et du Ministre chargé de l'Environnement.

ARTICLE 28 : Les établissements classés visés à l'article 26 ci-dessus, sont assujettis au paiement d'une taxe unique perçue lors de toute autorisation ou déclaration d'établissement classé. 
En outre, une redevance annuelle est perçue sur ceux dont la nature et/ou le volume des activités, font courir des risques particuliers à l'environnement, la santé et la sécurité publique et qui requièrent de ce fait des contrôles approfondis et périodiques.

Un décret pris en Conseil des Ministres sur proposition conjointe des Ministres chargés de l'Environnement, des Finances et de l'Administration du Territoire précise les modalités de répartition des produits de la taxe unique et/ou de la redevance entre l'Etat et les collectivités territoriales.

ARTICLE 29 Le taux de la taxe unique et/ou de la redevance annuelle est fixé par décret pris en Conseil des Ministres, sur proposition des Ministres concernés.

ARTICLE 30 : Lorsque l'installation, la construction, l'exploitation ou le fonctionnement d'un établissement industriel, artisanal, agricole ou de toute entreprise ou activité ou de tout engin, présentent pour le voisinage, la santé et la sécurité publique, des dangers ou des inconvénients graves, le Ministre chargé de l'Environnement peut, sur décision administrative, ordonner la fermeture de l'établissement ou de l'entreprise ou la suspension de l'activité sans préjudice des sanctions pénales applicables.

\section{SECTION 2 - DES MESURES SUR LES DECHETS URBAINS ET RURAUX}

ARTICLE 31 : Il est interdit de détenir ou d'abandonner des déchets urbains dans des conditions favorisant le développement d'animaux nuisibles, d'insectes et autres vecteurs de maladies susceptibles de provoquer des dommages aux personnes et aux biens.

ARTICLE 32 : Toute personne qui produit ou détient des déchets urbains dans des conditions susceptibles de porter atteinte à la santé et à la sécurité publique ou à l'environnement de façon générale, est tenue d'en assurer l'élimination conformément aux dispositions visées à l'article 33 ci-après.

ARTICLE 33: Dès l'entrée en vigueur du présent Code, les autorités locales chargées de la gestion des déchets urbains ainsi que toute autre personne physique ou morale concernée disposent de six (6) mois pour élaborer des plans de gestion des décharges et pollutions diverses à soumettre à l'avis préalable du Ministre chargé de l'Environnement avant leur mise en exécution.

ARTICLE 34 : Un décret pris en Conseil des Ministres, sur proposition du Ministre chargé de l'Environnement, en collaboration avec les Ministres concernés, réglemente la collecte, le stockage, le transport, le traitement et l'élimination des déchets urbains.

Des textes d'application pris à l'échelon local complètent les présentes dispositions.

ARTICLE 35: Les dispositions relatives à la gestion des déchets urbains visées aux articles 31, 32 et 33 ci-dessus, s'appliquent aux déchets de même nature produits en zone rurale.

\section{SECTION 3 - DES MESURES SUR LES DECHETS INDUSTRIELS OU ASSIMILES PRODUITS SUR LE TERRITOIRE NATIONAL}

ARTICLE 36 : Tout déchet industriel ou assimilé est dangereux dès lors qu'il présente une menace ou un danger quelconque pour la santé et la sécurité publique ou pour l'environnement, soit par lui-même, soit lorsqu'il entre en contact avec d'autres composés, du fait de leurs réactivités chimiques ou de leurs propriétés toxiques, explosives ou corrosives. 
ARTICLE 37 : Les établissements de 1ère et 2ème classes visés à l'article $\mathbf{2 5}$ ci-dessus, installés sur le Territoire National, sont soumis à un cahier des charges général, élaboré conjointement par les Ministères chargés de l'Industrie, des Mines, de l'Environnement, de la Santé, des Ressources hydrauliques, de l'Administration Territoriale et des Domaines.

Ce cahier des charges général, précise notamment les conditions générales d'élimination des déchets industriels, les conditions d'hygiène et de sécurité.

Sous réserve du respect des textes en vigueur, les collectivités territoriales, en relation avec les services techniques chargés de l'Environnement, des Mines, de la Santé, des Ressources hydrauliques et des Domaines, élaborent les cahiers des charges spécifiques qui prennent en compte les préoccupations particulières de leurs localités.

ARTICLE 38 : Le Ministère chargé de l'Environnement élabore en collaboration avec les Ministères concernés, un cahier des charges sectoriel qui précise les conditions matérielles et les techniques de stockage, de traitement, de recyclage, de transport et d'élimination des déchets industriels et assimilés.

\section{SECTION 4 - DES MESURES SUR LES DECHETS DANGEREUX EN PROVENANCE DE L'ETRANGER}

ARTICLE 39 : Est interdit au Burkina Faso, tout acte relatif au transit, à l'importation, à l'achat, à la vente, au transport, au traitement, au dépôt et au stockage des déchets dangereux.

ARTICLE 40 : Aucune matière radioactive, aucun appareil mettant en oeuvre une telle matière, ne peut être introduit au Burkina Faso, sans autorisation préalable donnée par décret pris en Conseil des Ministres, sur proposition du Ministre concerné.

ARTICLE 41 : Un décret pris en Conseil des Ministres sur proposition du Ministre chargé de l'Environnement fixe les conditions de gestion de la matière et de l'appareil visés à l'article 40 ainsi que celles des déchets produits.

\section{SECTION 5 - DES MESURES SUR LES PESTICIDES ET LES MATIERES FERTILISANTES.}

ARTICLE 42 : L'importation et la fabrication au Burkina Faso des pesticides et des matières fertilisantes sont soumises à autorisation et homologation préalables des Ministres chargés du Commerce, de l'Agriculture et de l'Elevage.

ARTICLE 43 : Sont interdites au Burkina Faso, la vente, la distribution à titre gratuit et l'utilisation des produits visés à l'article $\mathbf{4 2}$ ci-dessus, lorsque ceux-ci n'ont pas fait l'objet d'homologation ou lorsqu'ils sont périmés ou obsolètes.

ARTICLE 44 : Un décret pris en Conseil des Ministres sur proposition du Ministre chargé de l'Environnement fixe les conditions de transport, de stockage et d'utilisation des produits visés à l'article $\mathbf{4 2}$ ci-dessus, en vue de prévenir tout risque pour la santé et la sécurité publique et pour l'environnement.

ARTICLE 45 : La liste des pesticides et des matières fertilisantes prohibés au Burkina Faso est établie et mise à jour par le Ministre chargé de l'Agriculture et de l'Elevage.

ARTICLE 46 : Les stocks des produits visés à l'article 42 ci-dessus, lorsqu'ils sont périmés ou obsolètes sont détruits sans délai par le détenteur ou le propriétaire, sous le contrôle des services techniques chargés de l'Environnement.

Les frais de cette destruction sont à la charge du propriétaire ou du détenteur. 


\section{SECTION 6 - DES MESURES SUR LES POLLUTIONS ATMOSPHERIQUES}

ARTICLE 47 : Les immeubles, établissements industriels, commerciaux, artisanaux et agricoles, les mines et carrières, les véhicules à moteur ou autres objets mobiliers possédés, exploités ou détenus par toute personne physique ou morale doivent être construits, exploités ou utilisés de manière à satisfaire aux dispositions prises en application du présent Code, afin d'éviter les pollutions de l'atmosphère et les odeurs qui incommodent la population, compromettent la santé, la sécurité publique, ou nuisent à la production agricole et animale, à la conservation des sites et monuments.

ARTICLE 48 : Les conditions de construction, d'exploitation, d'utilisation des immeubles, établissements industriels, commerciaux, artisanaux et agricoles, des mines et carrières, des véhicules à moteur, engins et autres objets mobiliers sont déterminées par des décrets pris en Conseil des Ministres sur proposition des Ministres compétents.

Ces décrets précisent :

1) les conditions dans lesquelles peut être interdite ou réglementée l'émission dans l'atmosphère de fumées, suies, poussières ou gaz toxiques, corrosifs, odorants ou radioactifs;

2) les délais dans lesquels les immeubles, établissements, véhicules à moteur et autres objets mobiliers existant à la date de publication de chaque décret doivent satisfaire à ses obligations ;

3) les conditions dans lesquelles sont réglementés et contrôlés, aux fins prévues par l'article 47 ci-dessus, les constructions des immeubles, l'ouverture des établissements classés, l'équipement des véhicules à moteur, la fabrication des objets mobiliers et l'utilisation des combustibles et carburants ;

4) les conditions dans lesquelles l'administration peut, avant l'intervention des condamnations pénales, prendre, en raison de l'urgence, toutes les mesures exécutoires destinées à faire cesser d'office la pollution ;

5) les personnes qui peuvent être considérées comme pénalement responsables des infractions commises par des organismes de droit public.

\section{SECTION 7 - DES MESURES SUR LA POLLUTION DES EAUX ET DES SOLS}

ARTICLE 49 : Sont réglementés, tous les rejets, déversements, dépôts et toute activité susceptibles de provoquer à court, moyen et long termes, une dégradation de la qualité des sols et des eaux de surface ou souterraines.

Le Ministère chargé de l'Environnement et les départements ministériels concernés, élaborent conjointement, les normes de rejet ou de dépôt spécifiques qui prennent en compte les exigences du milieu récepteur, la qualité de l'environnement et les considérations socio-économiques, culturelles et techniques.

Ces normes qui sont révisées périodiquement, servent de base à l'élaboration des autorisations spéciales de rejet ou de dépôt.

L'avis des autorités locales est requis.

Sous réserve des textes en vigueur, les autorités locales édictent des mesures spécifiques de rejet ou de dépôt, prenant en compte les réalités biophysiques, économiques, sociales et culturelles particulières à leurs localités.

ARTICLE 50 : Les rejets, déversements, dépôts et toute activité tels que visés à l'alinéa premier de l'article 49 ci-dessus qui ne bénéficient pas d'autorisation spéciale sont interdits.

44 Textes et textes de loi sur la gestion des ressources naturelles au Burkina Faso 
ARTICLE 51: Les rejets ou dépôts qui ne font pas l'objet d'interdiction ni de soumission à autorisation préalable ni de règlement, demeurent libres, sous réserve que les conditions dans lesquelles ils sont effectués, la nature et les quantités de matières rejetées et/ou déposées ne soient pas susceptibles de :

- remettre en cause les usages qui sont faits de l'eau et du sol ;

- altérer les caractéristiques physico-chimiques et biologiques des milieux récepteurs ;

- nuire aux animaux, aux végétaux et à leur consommation ;

- porter atteinte à la santé et à la sécurité publique.

ARTICLE 52: Lorsque le rejet d'une matière fait l'objet d'une interdiction, les Ministres chargés de l'Environnement, des Ressources Hydrauliques, de l'Agriculture, de l'Elevage, de l'Industrie, de la Santé, de l'Administration du Territoire et de la Sécurité, interdisent ou réglementent conjointement la fabrication, l'importation, la détention, la vente et l'utilisation de cette matière.

L'interdiction et/ou la réglementation concerne également les produits entrant dans la composition des matières visées à l'alinéa 1 du présent article et les matériels conçus pour leur utilisation.

ARTICLE 53 : Les autorisations spéciales de rejet ou de dépôt précisent :

1) la dénomination des matières dont le rejet ou le dépôt est autorisé ;

2) le lieu de rejet ou de dépôt ;

3) la quantité globale du rejet ou du dépôt ;

4) la quantité par unité de temps ou de surface ainsi que toutes les prescriptions techniques nécessaires pour supprimer ou réduire les effets nocifs que le rejet ou le dépôt autorisé peut avoir sur le milieu récepteur, les êtres vivants, l'alimentation et la santé publique ;

5) la date limite de validité de l'autorisation et le montant de la taxe.

ARTICLE 54: Les bénéficiaires des autorisations spéciales de rejet ou de dépôt sont soumis à l'obligation de fournir des renseignements statistiques et de prendre toutes mesures utiles pour faciliter le contrôle des rejets et des dépôts.

ARTICLE 55 : Les autorisations de rejet ou de dépôt sont établies à titre individuel et leur délivrance est conditionnée par le paiement d'une taxe dans les conditions fixées par décret pris en Conseil des Ministres, sur proposition des Ministres concernés.

Ce décret détermine en outre, les modalités de répartition des produits de cette taxe entre l'Etat et les collectivités territoriales concernées.

ARTICLE 56 : Les Ministères chargés de l'Environnement, des Ressources Hydrauliques, de la Santé, de l'Administration du Territoire, de la Sécurité et de l'Industrie, désignent conjointement, les autorités investies du pouvoir de délivrer des autorisations spéciales de rejet ou de dépôt et définissent la limite de leur compétence.

Les Ministères concernés peuvent suspendre les autorisations de rejet ou de dépôt en cours de validité ou les rejeter par une décision motivée.

Aucune compensation ne peut être effectuée au profit du bénéficiaire d'une autorisation pour les préjudices dus à la suspension ou au retrait de cette autorisation.

\section{SECTION 8 - DES MESURES SUR LES PAYSAGES, LES SITES ET LES} MONUMENTS.

ARTICLE 57 : Il est interdit de détruire les sites, les paysages et les monuments présentant un intérêt scientifique, culturel ou historique. 
Un décret pris en Conseil des Ministres sur proposition des Ministres concernés et après concertation avec les Autorités locales, fixe la liste des sites, paysages et monuments à préserver. Cette liste est revue chaque fois que de besoin.

\section{CHAPITRE III : DES MESURES SUR L'AMELIORATION DU CADRE DE VIE SECTION 1 - DE L'ASSAINISSEMENT}

ARTICLE 58 : L'assainissement du cadre de vie est d'intérêt général.

Un décret pris en Conseil des Ministres sur proposition du Ministre chargé de l'Environnement, après concertation avec les Ministres concernés et les Autorités locales, définit une Stratégie Nationale d'Assainissement.

Ce décret précise notamment les modalités de mise en oeuvre, de suivi et d'évaluation de cette stratégie.

\section{SECTION 2 - DES NUISANCES DIVERSES}

ARTICLE 59 : L'interdiction ou la réglementation des nuisances diverses font l'objet de décrets pris en Conseil des Ministres sur proposition du Ministre chargé de l'Environnement, après concertation avec les parties concernées.

Ces décrets précisent les conditions de réglementation ou d'interdiction selon les lieux ou les moments, les responsabilités des personnes physiques ou morales publiques ou privées.

\section{SECTION 3 - DES AMENAGEMENTS PAYSAGERS}

ARTICLE 60 : Les embellissements qui font l'objet d'aménagements paysagers regroupent:

- les espaces verts ;

- les plantations d'alignement ;

- les jardins ;

- les ceintures vertes;

- les parterres ;

- les parcs urbains ;

- les squares ;

- les monuments ;

- les embellissements des sites, des monuments et des voies publiques.

ARTICLE 61 : Un décret pris en Conseil des Ministres sur proposition du Ministre chargé de l'Environnement en collaboration avec les Ministres concernés et après concertation avec les Autorités locales, définit une Stratégie Nationale d'Aménagements Paysagers et fixe les conditions d'attribution et d'exploitation de ces aménagements paysagers.

ARTICLE 62 : Les aménagements paysagers sont entrepris par des personnes physiques ou morales, de droit public ou privé, sur autorisation des autorités locales conformément aux dispositions du décret visé à l'article 61 ci-dessus.

ARTICLE 63 : Les projets d'aménagements paysagers d'intérêt public sont soumis à l'examen des services techniques compétents et leurs exécutions font l'objet de contrôles réguliers.

ARTICLE 64 :Un cahier des charges établi par l'autorité locale précise les conditions d'occupation et d'exploitation des aménagements paysagers à caractère public. 


\section{TITRE III - DE LA REPRESSION DES INFRACTIONS}

\section{CHAPITRE I : DES PROCEDURES}

\section{SECTION 1 - DE LA RECHERCHE ET DE LA CONSTATATION DES INFRACTIONS}

ARTICLE 65 : Ont compétence pour rechercher et/ou constater les infractions aux dispositions du présent Code :

- les officiers de police judiciaire ;

- les agents de police judiciaire ;

- les agents assermentés des Eaux et Forêts ;

- les agents assermentés des services de l'Hygiène et de l'Assainissement, de l'Agriculture et de l'Elevage, de l'Inspection du Travail ;

- les agents municipaux assermentés, chargés de la protection de l'Environnement et/ou de la Sécurité ;

- les agents assermentés de l'inspection économique ;

- tous autres agents assermentés, mandatés par le Ministre chargé de l'Environnement.

ARTICLE 66 : Les officiers de police judiciaire et les agents assermentés des Eaux et Forêts peuvent :

- pénétrer dans les enceintes et les bâtiments des exploitations industrielles ou agricoles, ainsi que dans les dépôts, entrepôts, magasins, lieux de stockage ou de vente des produits de ces exploitations ;

- inspecter les installations, aménagements, ouvrages, machines, véhicules, appareils et produits ;

- avoir accès à tout document relatif au fonctionnement de l'exploitation ou de l'entreprise commerciale;

- opérer des prélèvements, mesures, relevés d'analyses requis.

Les agents assermentés des autres services et les agents visés à l'article 65 ci-dessus doivent être accompagnés par un officier de police judiciaire ou par un agent assermenté des Eaux et Forêts.

Les agents visés à l'article $\mathbf{6 5}$ ci-dessus procèdent aux constats, enquêtes et perquisitions conformément aux dispositions du Code de Procédure Pénale et en dressent procèsverbal.

ARTICLE 67 : Les procès-verbaux contiennent l'exposé précis des faits et de toutes les circonstances pertinentes ainsi que les identités et déclarations des parties et des témoins s'il y a lieu.

Ces procès-verbaux valent à titre de simples renseignements. Ils font foi jusqu'à inscription de faux.

ARTICLE 68 : Le prévenu qui veut s'inscrire en faux contre un procès-verbal est tenu de le faire par écrit, au moins huit (8) jours avant l'audience indiquée par la citation.

ARTICLE 69: Les agents non assermentés des Eaux et Forêts et des autres services visés à l'article 65 ci-dessus ne peuvent établir que des rapports qui font foi jusqu'à preuve contraire. 


\section{SECTION 2 - DES ACTIONS ET DES POURSUITES}

ARTICLE 70 : Les conditions d'attribution de compétence des juridictions sont celles du Code Pénal.

ARTICLE 71 : Les actions et les poursuites devant les juridictions territorialement compétentes sont exercées par le Ministère chargé de l'Environnement sans préjudice du droit qui appartient au Ministère Public près ces juridictions.

ARTICLE 72 : Le Ministère chargé de l'Environnement, le Ministère Public, le prévenu et la partie civile peuvent relever appel des jugements relatifs aux infractions en matière de préservation de l'environnement rendus en premier ressort dans les conditions prévues par le Code Pénal.

\section{SECTION 3 - DES SAISIES ET DES CONFISCATIONS.}

ARTICLE 73 : Tout produit ou denrée est saisi ou confisqué dans le cadre de la répression des infractions commises en violation des dispositions du présent code, sans préjudice des sanctions pénales applicables.

ARTICLE 74 : En cas de saisie ou de confiscation de produit ou denrée, le procès-verbal de constatation des infractions porte mention desdites saisies ou confiscations.

ARTICLE 75 : Les produits et denrées provenant des confiscations sont vendus s'il y a lieu, par voie d'enchères publiques.

\section{SECTION 4 - DES TRANSACTIONS}

ARTICLE 76 : Dans le cadre de la répression des infractions commises en violation des dispositions du présent Code, le Ministère chargé de l'Environnement a la possibilité de transiger.

ARTICLE 77 : Le montant des transactions doit être acquitté dans les délais fixés dans l'acte des transactions, faute de quoi, il est procédé aux poursuites judiciaires.

ARTICLE 78 : Un décret pris en Conseil des Ministres sur proposition du Ministre chargé de l'Environnement fixe les barèmes des transactions applicables aux infractions commises en violation des dispositions du présent code.

\section{CHAPITRE II - DES INFRACTIONS ET DES SANCTIONS \\ SECTION 1 - DES INFRACTIONS EN MATIERE DE PRESERVATION DE L'ENVIRONNEMENT}

ARTICLE 79 : Quiconque met en chantier des activités en violation des dispositions de l'article 9 ci-dessus, est mis en demeure de suspendre lesdites activités, sans préjudice de la remise en état des lieux, à sa charge, dans un délai de trois (3) mois.

Passé ce délai, il lui est fait application d'une pénalité de retard de cent mille francs $(100.000 \mathrm{~F})$ par jour.

ARTICLE 80 : Est puni d'une peine d'emprisonnement de deux (2) mois à deux (2) ans et d'une amende de un million à cinq millions de francs (1.000.000 à 5.000.000 F) ou de l'une de ces deux peines seulement, quiconque importe, met en circulation, fabrique, distribue à titre gratuit ou vend un produit ou une denrée qui n'a pas été soumis au contrôle de qualité visé à l'alinéa ler de l'article $\mathbf{1 0}$ du présent code.

Il en est de même pour tout élément constitutif de l'environnement introduit ou émis en violation du même article. 
Le produit ou la denrée ainsi incriminé (e) est confisqué (e).

ARTICLE 81 : Le non paiement de la redevance visée à l'article 14 du présent code entraîne une pénalité équivalente au double de la redevance elle-même. En cas de mise en demeure restée sans effet, le coupable est puni d'une peine d'emprisonnement de trois (3) mois à un (1) an et d'une amende de un million à cinq millions de francs $(1.000 .000$ à 5.000.000 F) ou de l'une de ces deux peines seulement.

Le Tribunal saisi peut en outre ordonner la cessation de l'activité concernée.

ARTICLE 82 : Il est interdit d'entreprendre des activités en violation des dispositions des articles 15 et 17 ci-dessus.

ARTICLE 83 La violation des prescriptions visées à l'article 82 ci-dessus entraîne la suspension des activités ou la fermeture de l'établissement concerné par le service chargé de l'Environnement ou la juridiction saisie.

Il en est de même pour le non respect des prescriptions à l'article $\mathbf{1 7}$ ci-dessus.

ARTICLE 84 : Le non paiement de la taxe ou de la redevance visée à l'article 28 cidessus, entraîne une pénalité équivalente au double de la somme fixée.

En cas de mise en demeure restée sans effet, le coupable est puni d'une peine d'emprisonnement de un (1) mois à un (1) an et d'une amende de un million à cinq millions de francs $(1.000 .000$ à $5.000 .000 \mathrm{~F})$ ou de l'une de ces deux peines seulement.

Toute fausse déclaration susceptible d'influencer à la baisse la classification visée à l'article 26 ci-dessus et la fixation de la taxe ou de la redevance visée aux articles 14 et 28 ci-dessus, est punie d'une pénalité correspondant au double des sommes normalement fixées.

Le Tribunal saisi peut en outre ordonner la cessation de l'activité concernée.

ARTICLE 85 : Est puni d'une amende de cinquante mille à un million de francs (50.000 à 1.000.000 F) quiconque détient, produit ou abandonne des déchets urbains tels que définis à l'alinéa 7 de l'article $\mathbf{5}$ ci-dessus, en violation des dispositions des articles 31 et 32 ci-dessus.

ARTICLE 86 : Est puni d'une peine d'emprisonnement de un (1) à trois (3) ans et d'une amende de un million à dix millions de francs (1.000.000 à 10.000.000 F) ou de l'une de ces deux peines seulement, quiconque procède à l'installation, à l'ouverture, à la mise en exploitation ou à l'exploitation des établissements de première et deuxième classes visés à l'article 25 ci-dessus, en violation des dispositions des articles 37 et 38 ci-dessus.

ARTICLE 87 : Est puni d'une peine d'emprisonnement de dix (10) à vingt (20) ans et d'une amende de un milliard à cinq milliards de francs (1.000.000.000 à 5.000.000.000

F) ou de l'une de ces deux peines seulement, quiconque se livre à la manipulation des déchets dangereux, en violation des dispositions de l'article 39 ci-dessus.

La même peine est applicable à quiconque introduit ou utilise au Burkina Faso une matière radioactive ou un appareil mettant en œuvre une telle matière, en violation des dispositions de l'article $\mathbf{4 0}$ ci-dessus.

Toute tentative est punie comme l'infraction elle-même.

Les dispositions relatives au sursis ne sont pas applicables aux infractions prévues au présent article.

ARTICLE 88 : Les peines prévues à l'article 87 ci-dessus sont prononcées même si les divers actes qui constituent les éléments de l'infraction sont accomplis dans des pays différents, nonobstant les dispositions du Code Pénal relatives aux crimes et délits commis à l'étranger. 
Le tribunal saisi peut en outre ordonner la confiscation de la matière ou de l'appareil en cause au profit de l'Etat burkinabè.

ARTICLE 89 : Est puni d'une peine d'emprisonnement de un (1) à trois (3) ans et d'une amende de un million à dix millions de francs (1.000.000 à 10.000.000 F) ou de l'une de ces deux peines seulement, quiconque procède à l'importation, à la fabrication, à la distribution à titre gratuit, à la vente ou à l'utilisation des produits visés aux alinéas 10, et 11 de l'article 5 ci-dessus, en violation des dispositions des articles 42, 43 et 44 du ci-dessus.

ARTICLE 90 : Est puni d'une peine d'emprisonnement de un (1) à trois (3) ans et d'une amende de un million à cinq millions de francs (1.000.000 à 5.000.000 F) ou de l'une de ces deux peines seulement, quiconque contrevient aux dispositions de l'article 46 cidessus.

ARTICLE 91 : Est puni d'une peine d'emprisonnement de un (1) à trois (3) ans et d'une amende de un million à dix millions de francs (1.000.000 à 10.000.000 F) ou de l'une de ces deux peines seulement, quiconque contrevient aux dispositions de l'article $\mathbf{4 8}$ cidessus.

ARTICLE 92 : Est puni d'une peine d'emprisonnement de un (1) à trois (3) ans et d'une amende de un million à dix millions de francs (1.000.000 à 10.000.000 F) ou de l'une de ces deux peines seulement, quiconque contrevient aux dispositions des articles 49, 50 et 51 ci-dessus.

ARTICLE 93 : Est puni d'une peine d'emprisonnement de deux (2) à cinq (5) ans et d'une amende de un million à dix millions de francs (1.000.000 à 10.000.000 F) ou de l'une de ces deux peines seulement, quiconque contrevient aux dispositions de l'article 57 ci-dessus.

ARTICLE 94 : Dans le cadre de la répression des infractions prévues à la présente section, la juridiction saisie peut dans tous les cas ordonner la publication de la condamnation.

Elle peut en outre ordonner la remise en état et l'assainissement des lieux s'il y a lieu, sans préjudice des réparations civiles.

\section{SECTION 2 - DES INFRACTIONS EN MATIERE D'AMELIORATION DU CADRE DE VIE}

ARTICLE 95 : Est puni d'un emprisonnement de trois (3) mois à un (1) an et d'une amende de cent mille à un million de francs (100.000 à $1.000 .000 \mathrm{~F})$ ou de l'une de ces deux peines seulement, quiconque contrevient aux dispositions du décret visé à l'article 58 ci-dessus.

ARTICLE 96 : Est puni d'un emprisonnement de un (1) à six (6) mois et d'une amende de cinquante mille à cinq millions de francs $(50.000$ à 5.000.000 F) ou de l'une de ces deux peines seulement, quiconque est à l'origine d'une nuisance telle que définie à l'alinéa 16 de l'article 5 ci-dessus, en violation des dispositions de l'article 59 ci-dessus.

ARTICLE 97 : Est puni d'un emprisonnement de six (6) mois à un (1) an et d'une amende de cinq cent mille) cinq millions de francs (500.000 à 5.000.000 F) ou de l'une de ces deux peines seulement, quiconque entreprend, occupe ou exploite des aménagements paysagers en violation des dispositions des articles 62 et 64 du présent code. 
ARTICLE 98 : Dans tous les cas, la juridiction saisie peut ordonner la cessation ou la suspension des activités, la fermeture de l'établissement, la remise en état et l'assainissement des lieux.

Elle peut en outre ordonner la publication de la condamnation.

\section{SECTION 3 - DES INFRACTIONS AUX DISPOSITIONS TRANSITOIRES}

ARTICLE 99 : Est puni d'un emprisonnement de six (6) mois à un (1) an et d'une amende de un million à cinq millions de francs (1.000.000 à 5.000.000 F) ou de l'une de ces deux peines seulement, quiconque contrevient aux dispositions des articles 100, 102 et 103 ci-dessous.

\section{TITRE IV - DES DISPOSITIONS TRANSITOIRES}

ARTICLE 100 : A la date de promulgation de la loi, objet du présent Code, il est accordé un délai de deux (2) ans à tout promoteur ou exploitant de grands travaux, ouvrages, et aménagements déjà exécutés et ceux en cours de réalisation ou d'exploitation sans Etude ou Notice d'Impact sur l'Environnement de faire un audit environnemental à ses frais.

ARTICLE 101 : Les conditions de réalisation de l'audit environnemental visé à l'article 100 ci-dessus sont précisés par décret pris en Conseil des Ministres sur proposition du Ministre chargé de l'Environnement.

ARTICLE 102 : A la date d'entrée en vigueur du présent Code, tous les établissements, projets et activités susceptibles d'avoir des effets significatifs sur l'environnement dans un délai de six (6) mois.

ARTICLE 103 : Il est fait obligation de détruire immédiatement sous le contrôle des services techniques compétents chargés de l'Environnement, les stocks de produits périmés ou obsolètes, conformément aux dispositions du présent Code.

Les frais de destruction sont à la charge du propriétaire ou du détenteur.

\section{TITRE V - DES DISPOSITIONS FINALES}

ARTICLE 104 : La présente loi qui abroge toutes dispositions antérieures contraires, notamment la loi $\mathbf{n}^{\circ}$ 002/94/ADP du 19 janvier 1994, sera exécutée comme loi de l'Etat.

Ainsi fait et délibéré en séance publique à Ouagadougou, le 30 janvier 1997

Pour le Président,

Deuxième Vice-Président

Jean-Marie SOMDA

Le Secrétaire de Séance

Batio Isaïe TRAORE 


\section{Annexe IV}

\section{LOI Nº06/97/ADP PORTANT CODE FORESTIER AU BURKINA FASO}

\section{L'ASSEMBLEE DES DÉPUTÉS DU PEUPLE}

$\mathrm{Vu}$ la Constitution ;

$\mathrm{Vu}$ la Résolution n 001/92/ADP du 17 juin 1992, portant validation du mandat des Députés ;

A délibéré en séance du 31 janvier 1997

et adopté la loi dont la teneur suit :

\section{TITRE PRÉLIMINAIRE - DES DISPOSITIONS GENERALES}

CHAPITRE 1 : DE L'OBJET ET DU BUT

ARTICLE 1 : Le présent code a pour objet de fixer, conformément à la politique forestière nationale, l'ensemble des principes fondamentaux relatifs à la conservation et à la gestion durable des ressources naturelles forestières, fauniques et halieutiques.

ARTICLE 2: Il vise en particulier à établir une articulation harmonieuse entre la nécessaire protection de ces ressources et la satisfaction des besoins économiques, culturels et sociaux de la population.

ARTICLE 3 : Le code définit les conditions favorables au développement performant des sous-secteurs des forêts, de la faune et des pêches, tout en assurant la promotion des principes de gestion participative et durable des ressources concernées.

\section{CHAPITRE 2 : DU RÉGIME GÉNÉRAL}

ARTICLE 4 : Les forêts, la faune et les ressources halieutiques constituent des richesses naturelles et sont à ce titre, conformément à la constitution, parties intégrantes du patrimoine national.

Elles doivent être protégées dans l'intérêt de l'humanité et valorisées en vue de l'amélioration des conditions de vie de la population. Chacun a le devoir de respecter ces éléments du patrimoine national, et de contribuer à leur conservation.

ARTICLE 5 : L'Etat est garant de la préservation des ressources forestières, fauniques et halieutiques. Il exerce cette responsabilité à travers les services techniques forestiers, en concertation avec l'ensemble des acteurs concernés par l'utilisation, l'exploitation et la gestion des ressources naturelles.

\section{CHAPITRE 3 : DE LA POLITIQUE FORESTIÈRE NATIONALE}

ARTICLE 6 : Les forêts, la faune et les ressources halieutiques font l'objet d'une politique nationale, ci-après dénommée politique forestière nationale.

La politique forestière nationale garantit une action concertée et complémentaire de l'ensemble des institutions et structures concernées afin de réaliser les objectifs globaux définis par le gouvernement.

ARTICLE 7 : La politique forestière nationale est fondée sur les principales options fondamentales suivantes : 
- la conservation de la diversité biologique ;

- la valorisation des ressources forestières, fauniques et halieutiques pour le développement économique et l'amélioration du cadre de vie ;

- la génération d'emplois et de revenus au profit de la population ;

- la participation et la responsabilisation effectives de la population dans la conception, l'exécution, le suivi et l'évaluation des activités forestières, notamment à travers la gestion décentralisée des ressources naturelles.

ARTICLE 8: Il est institué un fonds forestier affecté au financement des actions d'entretien, de régénération et de conservation des ressources forestières, fauniques et halieutiques.

ARTICLE 9 : Le fonds forestier est alimenté par :

- les dotations de l'Etat ;

- les concours financiers des institutions de coopération bilatérale et multilatérale ;

- les dons et legs des personnes physiques ou morales de droit privé ;

- toutes autres recettes définies par la loi de finances.

ARTICLE 10 : Les textes d'application du présent code préciseront l'organisation et les modalités de fonctionnement de ce fonds.

\section{LIVRE PREMIER - DES FORETS}

\section{TITRE I - DU DOMAINE FORESTIER}

ARTICLE 11 : Le domaine forestier comprend les forêts publiques et les forêts privées.

\section{CHAPITRE 1 : DES DÉFINITIONS}

ARTICLE 12 : Au sens du présent code, sont considérés comme forêts les espaces occupés par des formations végétales d'arbres et d'arbustes, à l'exclusion de celles résultant d'activités agricoles.

ARTICLE 13 : Sont soumis au régime forestier les périmètres de restauration et les périmètres de reboisement.

ARTICLE 14 : Les périmètres de restauration sont des portions de terrains dégradés, délimités en vue de la réalisation d'opérations de régénération.

Les périmètres de reboisement sont des espaces déboisés, délimités pour être enrichis en arbres.

ARTICLE 15 : Sont également soumis au régime forestier, les produits forestiers.

Les produits forestiers sont ceux provenant des formations végétales d'arbres et d'arbustes, ainsi que tout ce qui se trouve dans les limites de la forêt.

\section{CHAPITRE 2 : DES FORÊTS PUBLIQUES}

ARTICLE 16 : Les forêts publiques sont constituées par toutes les forêts telles que définies au chapitre premier du présent titre, qui ne font pas l'objet d'appropriation privée. Les forêts publiques sont classées ou protégées.

ARTICLE 17 : Les forêts publiques sont réparties entre le patrimoine de l'Etat et les patrimoines des collectivités territoriales décentralisées. 
Les modalités de répartition des forêts entre l'Etat et les collectivités territoriales décentralisées sont déterminées par la présente loi, ses textes d'application et par ceux régissant la composition des patrimoines des collectivités territoriales décentralisées.

\section{SECTION 1 : DU DOMAINE FORESTIER DE L'ETAT}

ARTICLE 18 : Le domaine forestier de l'Etat est constitué :

- des forêts classées au nom de l'Etat à la date d'entrée en vigueur de la présente loi et qui n'ont pas fait l'objet d'une procédure de déclassement ;

- des forêts classées au nom de l'Etat en application des dispositions du présent code et de ses textes d'application du présent code.

ARTICLE 19 : Toute forêt publique peut faire l'objet d'un classement au nom de l'Etat dans un but d'intérêt général national.

ARTICLE 20 : Relèvent de l'intérêt général national, les forêts dont notamment la taille, l'importance écologique, ou la valeur esthétique nécessitent des mesures ou des précautions de gestion qui dépassent les moyens et les capacités d'une seule collectivité territoriale décentralisée.

Relèvent également de l'intérêt général national, les forêts affectées à des buts de conservation, dont l'intérêt dépasse celui d'une seule collectivité territoriale décentralisée.

ARTICLE 21 : Relèvent de l'intérêt général national, et sont obligatoirement classés au nom de l'Etat, les réserves de la biosphère, les parcs nationaux, les réserves naturelles intégrales et les sanctuaires. Ces espaces sont soumis à des régimes spécifiques.

\section{SECTION 2 : DU DOMAINE FORESTIER DES COLLECTIVITÉS TERRITORIALES DÉCENTRALISÉES}

ARTICLE 22 : Le domaine forestier des collectivités territoriales décentralisées est composé de l'ensemble des forêts situées sur le territoire national, à l'exclusion de celles qui appartiennent à des personnes privées et de celles qui font l'objet d'un classement au nom de l'Etat.

ARTICLE 23 : Les forêts des collectivités territoriales décentralisées peuvent faire l'objet d'un acte de classement au nom de ces collectivités dans un but d'intérêt général local.

ARTICLE 24 : Relèvent de l'intérêt général local les forêts dont la taille, la valeur écologique ou esthétique, permettent une gestion rationnelle et durable avec les moyens et les capacités de la collectivité territoriale considérée.

Relèvent également de l'intérêt général local, les forêts affectées à des buts de conservation dont l'intérêt ne dépasse pas celui de la collectivité territoriale concernée.

\section{SECTION 3 : DU CLASSEMENT ET DU DÉCLASSEMENT}

ARTICLE 25: Conformément aux dispositions des articles 19 et 23 ci-dessus, les forêts peuvent être classées soit au nom de l'Etat, soit au nom des collectivités territoriales décentralisées.

ARTICLE 26 : Le classement forestier permet, en raison de l'importance qu'une forêt présente pour l'intérêt général, de soumettre celle-ci à un régime spécial restrictif concernant l'exercice des droits d'usage et les régimes d'exploitation.

Les forêts qui n'ont pas fait l'objet d'un acte de classement sont appelées forêts protégées ; elles sont soumises au régime commun relatif aux droits d'usage et d'exploitation. 
ARTICLE 27 : Tout acte de classement donne lieu à des opérations matérielles de délimitation et de signalisation sur le terrain, dans les conditions précisées par les textes d'application du présent code.

ARTICLE 28 : Sauf disposition législative contraire, le classement d'une forêt dans le domaine forestier de l'Etat résulte d'un décret pris en conseil des Ministres sur proposition du Ministre chargé des forêts.

Le classement d'une forêt dans le domaine forestier des collectivités territoriales décentralisées résulte d'un arrêté pris par l'autorité locale compétente, après avis du Ministre chargé des forêts.

ARTICLE 29 : L'acte de classement précise les objectifs du classement, la superficie, les limites exactes de la forêt, ses affectations principales ou exclusives et les modalités de sa gestion.

Les modalités des diverses phases de la procédure de classement sont précisées par les textes d'application dans le respect notamment de l'approche participative et de l'intégration des actions forestières dans le cadre global du développement rural.

ARTICLE 30 : Les actes de classement des forêts dans le domaine forestier de l'Etat et dans le domaine forestier des collectivités territoriales décentralisées peuvent être révisés.

Les procédures de révision des classements sont précisées par les textes d'application.

ARTICLE 31 : Le classement des forêts au nom de l'Etat ou des collectivités territoriales décentralisées n'est pas immuable. Les forêts classées au nom de l'Etat ou des collectivités territoriales décentralisées sont susceptibles de déclassement.

Les forêts classées au nom de l'Etat peuvent être déclassées au profit des collectivités territoriales décentralisées ; inversement, les forêts classées au nom des collectivités territoriales peuvent être déclassées au profit de l'Etat.

ARTICLE 32 : Le déclassement d'une forêt de l'Etat résulte d'un décret pris en Conseil des Ministres sur proposition du Ministre chargé des forêts.

Le déclassement d'une forêt d'une collectivité territoriale décentralisée résulte d'un arrêté de l'autorité locale compétente, pris après avis du Ministre chargé des forêts.

\section{CHAPITRE 3 : DES FORÊTS PRIVÉES}

ARTICLE 33 : Les personnes physiques ou morales de droit privé sont propriétaires des forêts qu'elles ont légalement acquises ou qu'elles ont légalement plantées.

Les personnes physiques ou morales de droit privé ne peuvent être propriétaires de forêts que moyennant la détention d'un titre régulier de jouissance sur le sol forestier.

ARTICLE 34 : Les forêts privées sont gérées librement par leurs pro-priétaires, sous réserve des déclarations d'exploitation et éventuellement des restrictions imposées pour la préservation du milieu naturel, en vertu des dispositions réglementaires prises en application du présent code.

\section{TITRE II - DE LA GESTION FORESTIERE}

ARTICLE 35 : La conservation, le développement et l'exploitation des forêts sont assurés par une gestion rationnelle et équilibrée. 
Les services forestiers de l'Etat sont garants de la préservation des ressources forestières considérées comme éléments du patrimoine national, conformément à l'article $4 \mathrm{du}$ présent code.

\section{CHAPITRE 1 : DES PRINCIPES DE GESTION}

ARTICLE 36 : La gestion forestière repose sur le principe de l'intégration de la protection, de l'exploitation et de la valorisation du patrimoine forestier.

Elle garantit la préservation du milieu naturel au profit des générations futures, tout en assurant la satisfaction des besoins socio-économiques et culturels des générations présentes.

ARTICLE 37 : Les forêts sont gérées sous le contrôle de l'Etat ou de la collectivité territoriale décentralisée, dans le respect de la réglementation en vigueur et de l'approche participative et concertée.

ARTICLE 38 : Le domaine forestier de l'Etat est géré par les services forestiers de l'Etat. Toutefois, la gestion de ce domaine forestier peut être confiée à des tiers dans les conditions prévues par la présente loi.

ARTICLE 39 : Les services forestiers peuvent par contrat, confier l'exploitation d'une partie du domaine forestier de l'Etat à une personne physique ou morale, qu'elle soit de droit privé ou de droit public. Le contrat est assorti d'un cahier des charges qui précise les conditions de l'exploitation et les modalités de répartition des produits.

Les modalités de gestion de ces forêts sont déterminées par les textes d'application qui doivent prévoir dans la mesure du possible, des avantages au profit des populations riveraines.

ARTICLE 40 : La gestion des forêts des collectivités territoriales décentralisées est assurée par ces dernières à travers des structures de gestion fondées sur le partenariat. La création de ces structures est réalisée par arrêté de l'autorité compétente de la collectivité territoriale décentralisée.

ARTICLE 41 : La gestion des forêts se fait conformément aux prescriptions des plans d'aménagement forestier.

Les plans d'aménagement forestier sont élaborés par les services forestiers ou sous leur contrôle. Ils sont approuvés par arrêté du Ministre chargé des forêts lorsqu'ils concernent des forêts de l'Etat, et par arrêté de l'autorité compétente de la collectivité territoriale décentralisée lorsqu'ils concernent des forêts des collectivités territoriales décentralisées.

ARTICLE 42: Le plan d'aménagement forestier peut être révisé si des conditions nouvelles concernant l'unité aménagée l'exigent. La procédure de révision du plan est identique à celle de son élaboration.

\section{CHAPITRE 2 : DE LA PROTECTION DES FORÊTS SECTION 1 : DES DISPOSITIONS GÉNÉRALES}

ARTICLE 43 : Les forêts sont protégées contre toutes formes de dégradation et de destruction, qu'elles soient naturelles ou provoquées.

ARTICLE 44 : La protection des forêts incombe à l'Etat, aux collectivités territoriales décentralisées et aux communautés villageoises riveraines. 
ARTICLE 45 : La protection s'entend de l'ensemble des opérations d'entretien, de régénération et de conservation du patrimoine forestier. Elle implique le respect de la réglementation en vigueur des plans d'aménagement et des contrats de gestion.

Les services forestiers concourent au respect de cette obligation dans les conditions précisées par les textes d'application.

ARTICLE 46 : Certaines espèces forestières, en raison de leur intérêt ethno-botanique spécifique ou des risques de disparition qui les menacent, bénéficient de mesures de protection particulières. Leur liste est déterminée par arrêté du Ministre chargé des forêts.

ARTICLE 47 : L'introduction des espèces forestières exotiques sur le territoire national est soumise à une autorisation préalable du Ministre chargé des forêts.

ARTICLE 48 : Sur l'ensemble du domaine forestier, l'administration chargée des forêts est habilitée à prendre toutes mesures nécessitées par les conditions spécifiques du milieu et notamment la fixation des sols en pente, la protection des terres et des ouvrages contre l'action érosive, la conservation des espèces rares et des biotopes fragiles, la protection des sources et des cours d'eau.

\section{SECTION 2 : DU DÉFRICHEMENT}

ARTICLE 49 : Tout défrichement portant sur une portion de forêt supérieure à une superficie donnée fixée par voie réglementaire, est soumis à une autorisation préalable.

Les modalités et conditions de délivrance de cette autorisation préalable seront déterminées par les textes d'application.

ARTICLE 50 : Toute réalisation de grands travaux entraînant un défrichement est soumise à une autorisation préalable du Ministre chargé des Forêts sur la base d'une étude d'impact sur l'environnement.

ARTICLE 51 : Quel que soit le régime des forêts en cause, le Ministre chargé des forêts peut, par arrêté, déterminer des zones soustraites à tout défrichement en considération de leur importance particulière pour le maintien de l'équilibre écologique.

\section{SECTION 3 : DES FEUX DE BROUSSE}

ARTICLE 52 : Afin de prévenir les incendies de forêts, les feux de brousse sont prohibés en dehors du cadre défini par la législation en vigueur.

ARTICLE 53 : Lorsque des mises à feu précoces ou contrôlées de certaines zones sont utilisées comme instrument d'action et d'aménagement forestier, elles sont réalisées dans le strict respect de la réglementation en vigueur.

\section{CHAPITRE 3 : DE L'EXPLOITATION}

ARTICLE 54 : L'exploitation forestière s'entend des opérations visant à réaliser un profit économique grâce aux produits forestiers.

L'exploitation forestière peut être faite à des fins domestiques, commerciales ou industrielles.

\section{SECTION 1 : DE L'EXPLOITATION DOMESTIQUE}

ARTICLE 55 : L'exploitation forestière domestique s'exerce sous forme de droits d'usage traditionnels de cueillette ou de ramassage. 
ARTICLE 56 : Dans les forêts classées, les droits d'usage traditionnels sont reconnus aux populations riveraines; elles concernent le ramassage du bois mort gisant, la cueillette des fruits et la récolte des plantes médicinales.

ARTICLE 57 : Dans les forêts protégées les droits d'usage traditionnels reconnus au profit des populations riveraines portent sur la culture, le pâturage, la cueillette des produits et sous-produits forestiers.

ARTICLE 58 : Tous autres droits d'usage traditionnels peuvent être autorisés pour chaque forêt, par le plan d'aménagement forestier qui lui est applicable.

ARTICLE 59 : L'exercice des droits d'usage traditionnels est limité à la satisfaction des besoins personnels, individuels ou familiaux des usagers. Il se fait à titre gratuit et sans permis, dans le respect de la réglementation en vigueur ; il ne peut donner lieu à une exploitation commerciale.

\section{SECTION 2 : DE L'EXPLOITATION COMMERCIALE OU INDUSTRIELLE}

ARTICLE 60 : Toute exploitation forestière à des fins commerciales ou industrielles donne lieu à paiement de taxes et redevances.

ARTICLE 61 : Les exploitants sont tenus de se conformer aux prescriptions des plans d'aménagement forestier établis en vue de rationaliser la gestion des forêts, sur la base d'une conciliation des intérêts de la production et de ceux de la protection.

ARTICLE 62 : Les forêts sont exploitées soit directement par leurs propriétaires, soit par des exploitants non propriétaires, et selon les cas, sur la base d'une autorisation administrative, d'un contrat, ou en régie.

ARTICLE 63 : Dans un but de contrôle et de suivi des prélèvements de la forêt, un permis de coupe est exigé pour tout abattage d'arbre à l'intérieur d'une forêt, sauf celui effectué sur une exploitation agricole permanente effective.

ARTICLE 64: La délivrance des permis de coupe est subordonnée à l'acquittement d'une taxe dont le taux, l'assiette et les modalités de perception sont fixés par la loi de finances.

ARTICLE 65 : Les services forestiers prêtent leur assistance à titre gratuit ou onéreux selon les cas, aux exploitants qui le requièrent, notamment pour l'exécution de travaux forestiers ou la fourniture de conseils techniques.

Ils exercent un contrôle sur les conditions de l'exploitation forestière.

ARTICLE 66 : L'exploitation des forêts des collectivités territoriales décentralisées doit répondre à l'exigence de l'intégration de la foresterie dans le développement rural. Elle contribue à la gestion optimale et durable de l'ensemble des potentiels de production agricoles, pastoraux et forestiers.

ARTICLE 67 : En fonction de leur situation géographique et de la proximité des communautés villageoises, les forêts des collectivités territoriales décentralisées sont exploitées soit directement par ces collectivités, soit indirectement par les communautés villageoises concernées.

ARTICLE 68 : En application de l'approche participative qui sous-tend la politique forestière, les collectivités territoriales décentralisées peuvent transférer l'exploitation de leurs forêts aux communautés villageoises ou inter-villageoises relevant de leur ressort. 
ARTICLE 69 : Les modalités relatives au droit d'exploitation transféré par la collectivité territoriale décentralisée aux communautés villageoises et celles relatives au contrôle de l'exercice de ce droit sont précisées par contrat.

Les termes du contrat fixentl'étendue du pouvoir degestion des communautés, les conditions de la collaboration entre les communautés et les collectivités territoriales décentralisées, ainsi que les modalités de la mise en jeu de la responsabilité des communautés.

ARTICLE 70 : Le stockage et la circulation des produits forestiers à des fins commerciales sont soumis à autorisation préalable.

Le Ministre chargé des forêts et ceux chargés du transport et du commerce déterminent par voie d'arrêté conjoint les conditions de circulation et de stockage de ces produits.

\section{LIVRE II - DE LA FAUNE}

\section{TITRE I - DE LA PROTECTION DE LA FAUNE}

\section{CHAPITRE 1 : DES DÉFINITIONS ET DES PRINCIPES DE PROTECTION}

ARTICLE 71 : Au sens de la présente loi, la faune est l'ensemble des animaux sauvages, vivant en liberté dans leur milieu naturel, ou maintenus en captivité, à l'exception des poissons, des mollusques et des crustacés.

ARTICLE 72 : Les aires fauniques de protection sont des espaces spécialement réservés pour la conservation de la faune et de son habitat.

ARTICLE 73 : La protection de la faune vise la sauvegarde des différentes espèces de faune et de leurs habitats.

ARTICLE 74 : Tout animal sauvage se trouvant sur le territoire national bénéficie de la protection conférée à la faune par la présente loi, par les textes complémentaires et d'application, ainsi que par les conventions internationales ratifiées par le Burkina Faso.

ARTICLE 75 : Les animaux sauvages doivent être traités avec le respect dû par l'hommeà la nature ; aucune souffrance ni aucune destruction ne doit leur être infligée sans nécessité.

\section{CHAPITRE 2 : DES AIRES DE PROTECTION}

\section{SECTION 1 : DE LA DÉTERMINATION DES AIRES DE PROTECTION}

ARTICLE 76 : En vue d'assurer la protection des habitats de la faune, certaines parties du territoire national peuvent être classées et affectées à la constitution d'aires de protection faunique.

ARTICLE 77 : Les aires de protection faunique pouvant être créées sur le territoire du Burkina Faso sont notamment constituées par :

- les parcs nationaux ;

- les réserves de faune, totales ou partielles ;

- les réserves de la biosphère ;

- les sanctuaires ;

- les ranches;

- les refuges locaux ;

- les zones villageoises d'intérêt cynégétique. 
En application des conventions internationales dûment ratifiées par le Burkina Faso et, selon les besoins, il peut être créé d'autres types d'aires de protection faunique.

ARTICLE 78 : Chaque aire de protection faunique doit faire l'objet d'un plan d'aménagement approuvé par le Ministre chargé de la faune.

Les plans d'aménagement définissent notamment les infrastructures à réaliser et précisent les activités qui peuvent être menées à l'intérieur de l'aire de protection.

ARTICLE 79 : Dans la mesure du possible, toute aire de protection faunique doit comporter une zone tampon.

Une zone tampon est une bande périmétrale destinée à la réalisation d'aménagements spéciaux d'ordre économique, social ou culturel, compatibles avec les objectifs de l'aire protégée.

La zone tampon est partie intégrante de l'aire de protection.

ARTICLE 80 : Sauf dispositions législatives contraires, la procédure de classement et de déclassement des aires fauniques est celle applicable au domaine forestier de l'Etat.

\section{SECTION 2 : DES SANCTUAIRES ET DES RÉSERVES DE LA BIOSPHÈRE}

ARTICLE 81 : Une réserve de la biosphère est une aire déclarée comme bien du patrimoine mondial en raison de ses spécificités biologiques, écologiques, culturelles ou historiques particulières.

ARTICLE 82 : Un sanctuaire est une aire affectée à la protection de communautés caractéristiques de végétaux, d'animaux ou de sites particulièrement menacés.

ARTICLE 83 : La constitution des sanctuaires et des réserves de la biosphère relève du domaine de la loi.

ARTICLE 84 : La loi créant le sanctuaire ou la réserve de la biosphère détermine les conditions particulières de protection et d'aménagement de ces aires.

\section{SECTION 3 : DES PARCS NATIONAUX}

ARTICLE 85 : Un parc national est une partie du territoire national classée au nom de l'Etat en vue de la conservation de la flore, de la faune, des eaux, des sols, des paysages ou des formations géologiques ayant une valeur scientifique ou esthétique.

ARTICLE 86: La constitution des parcs nationaux relève du domaine de la loi.

ARTICLE 87 : Sauf cas exceptionnel, et sous réserve des dispositions du texte constitutif ou des prescriptions du plan d'aménagement, les parcs nationaux sont soustraits à tout droit d'usage.

Le texte constitutif du parc doit, le cas échéant, préciser les mesures compensatoires prévues au profit des populations locales concernées.

ARTICLE 88 : Sont prohibés, à l'intérieur des limites des parcs nationaux, le pâturage, les défrichements, la chasse, l'exploitation agricole, forestière ou minière, le dépôt des déchets, les activités polluantes, les feux incontrôlés et, en général, tout acte incompatible avec la conservation et la protection du milieu considéré.

Toutefois l'exercice de la pêche pourra être autorisé par les plans d'aménagements relatifs aux parcs nationaux.

60 Textes et textes de loi sur la gestion des ressources naturelles au Burkina Faso 
ARTICLE 89 : Les conditions particulières d'entrée, de circulation et de séjour dans un parc national ainsi que les modalités de participation des populations à sa gestion sont précisées par son texte constitutif.

ARTICLE 90 : Des protocoles d'accord peuvent être conclus entre l'état et les personnes physiques ou morales de droit privé en vue de l'aménagement et de la valorisation des parcs nationaux à des fins touristiques et culturelles.

\section{SECTION 4 : DES RÉSERVES DE LA FAUNE}

ARTICLE 91 : Les réserves de faune sont des aires classées au nom de l'Etat pour la conservation et la propagation de la vie sauvage ainsi que l'aménagement de l'habitat.

Les réserves de faune sont soit totales, soit partielles.

ARTICLE 92 : Les réserves totales de faune sont établies pour la protection de toutes les espèces de faune ; les activités de chasse y sont interdites.

Les réserves partielles de faune sont établies pour la protection particulière de certaines espèces ; les activités de chasse y sont autorisées.

ARTICLE 93 : La constitution des réserves totales de faune relève du domaine de la loi. La constitution des réserves partielles est faite par décret pris en Conseil des Ministres.

ARTICLE 94: Les redevances et taxes collectées dans le cadre de la valorisation des réserves de faune font l'objet d'une répartition entre le budget de l'Etat et celui des collectivités locales.

\section{SECTION 5 : DES REFUGES LOCAUX ET DES ZONES VILLAGEOISES D'INTÉRÊT CYNÉGÉTIQUE}

ARTICLE 95: Au sens de la présente loi, les refuges locaux sont des aires protégées, classées au nom des collectivités territoriales décentralisées et réservées par elles en vue de favoriser la reproduction, la propagation et l'exploitation d'espèces fauniques sur leur territoire, ainsi que la conservation des habitats nécessaires à la survie de ces espèces.

ARTICLE 96 : Les refuges locaux sont créés dans les mêmes conditions que les forêts classées des collectivités territoriales décentralisées.

ARTICLE 97 : La gestion des refuges locaux relève de la compétence des collectivités territoriales décentralisées, qui bénéficient à cet effet de l'assistance des services techniques locaux chargés de la faune.

Les collectivités décentralisées prennent toutes les mesures nécessaires en vue d'assurer la participation effective des représentants des communautés concernées à cette gestion. En particulier elles peuvent mettre en place des structures ad hoc de gestion fondée sur le partenariat, et dont la composition est déterminée par les textes complémentaires et d'application.

ARTICLE 98 : Les activités autorisées à l'intérieur des refuges locaux sont déterminées par les collectivités territoriales décentralisées avec l'assistance des services techniques locaux chargés de la faune.

ARTICLE 99 : Une zone villageoise d'intérêt cynégétique est une partie du terroir d'une communauté de base, affectée par elle à l'exploitation des ressources cynégétiques. Sa création est proposée par un procès-verbal de réunion de l'organe villageois compétent et confirmée par arrêté de l'autorité locale compétente. 
ARTICLE 100 : La gestion des zones villageoises d'intérêt cynégétique peut être assurée par des associations ou groupements villageois ou toute autre structure juridique dotée de la personnalité morale. Ces zones peuvent faire l'objet d'amodiation entre les communautés concernées et des professionnels de la faune pour l'organisation lucrative d'activités cynégétiques.

ARTICLE 101 : Les activités autorisées à l'intérieur des zones villageoises d'intérêt cynégétique sont déterminées par la communauté de base avec l'assistance des services techniques locaux chargés de la faune.

ARTICLE 102 : Les redevances et taxes collectées dans le cadre de la gestion des refuges locaux et des zones villageoises d'intérêt cynégétique sont réparties entre les budgets locaux et les organisations villageoises de gestion de la faune.

\section{CHAPITRE 3 : DES CATÉGORIES D'ESPÈCES FAUNIQUES SECTION 1 : DU PRINCIPE DE CLASSIFICATION}

ARTICLE 103 : Les espèces fauniques sont classées en deux catégories : les espèces intégralement protégées et les espèces partiellement protégées.

ARTICLE 104 : Tous les animaux relevant de la catégorie des espèces intégralement protégées font l'objet d'une inscription sur une liste de protection dite liste A.

Certains animaux de la catégorie des espèces partiellement protégées font l'objet d'une inscription sur une liste de protection dite liste B.

Au sens de la présente loi, sont appelées espèces non inscrites les animaux de la catégorie des espèces partiellement protégées, ne faisant pas l'objet d'une inscription sur la liste de protection.

ARTICLE 105 : Les listes A et B de protection sont adoptées par décret pris en conseil des ministres.

ARTICLE 106 : Afin d'assurer leur adaptation à l'évolution des populations animales, les listes de protection peuvent faire l'objet d'une révision périodique.

\section{SECTION 2 : DU RÉGIME APPLICABLE}

ARTICLE 107 : Les espèces intégralement protégées font l'objet d'une prohibition totale de prélèvement, que ce soit par capture, chasse ou ramassage d'œufs.

ARTICLE 108 : Les espèces partiellement protégées inscrites sur la liste B de protection sont soumises à un régime de prélèvement étroitement contrôlé, notamment par la fixation de latitudes d'abattage et de quotas.

ARTICLE 109 : Les espèces non inscrites bénéficient des mesures générales de sauvegarde de la faune prévues par la présente loi et par les conventions internationales ratifiées par le Burkina Faso.

ARTICLE 110 : Le Ministre chargé de la faune peut placer temporairement certains animaux de la liste des espèces partiellement protégées sous un régime de protection intégrale en cas de menace grave pesant sur eux ou sur leur habitat, ou encore en vue de favoriser leur reconstitution. Information large en est donnée au public, notamment aux associations de chasseurs, par tous moyens appropriés.

Ce régime dérogatoire de protection temporaire ne pourra excéder trois saisons de chasse.

62 Textes et textes de loi sur la gestion des ressources naturelles au Burkina Faso 
ARTICLE 111 : Sur proposition des autorités locales intéressées, l'organe compétent de la collectivité territoriale décentralisée concernée peut prendre des dispositions en vue d'assurer la protection intégrale ou partielle d'une espèce animale sauvage sur le territoire de ladite collectivité.

\section{TITRE II : DE L'EXPLOITATION DE LA FAUNE}

ARTICLE 112 : L'exploitation de la faune se réalise principalement par les actions suivantes : la chasse, la capture, la récolte, le ranching et le tourisme de vision.

\section{CHAPITRE 1 : DE LA CHASSE}

\section{SECTION 1 : DU DROIT DE CHASSE}

ARTICLE 113 : La chasse est tout acte tendant à tuer, blesser, poursuivre, rechercher, inquiéter, viser un animal en liberté ou à détruire, ramasser des oeufs d'oiseaux ou de reptiles.

ARTICLE 114 : Il est institué deux types de chasse : la chasse sportive et la chasse de subsistance ou chasse traditionnelle.

ARTICLE 115 : Au sens de la présente loi, la chasse sportive est celle exercée sans but lucratif par les détenteurs de permis de chasse sportive à des fins récréatives et sportives.

La chasse de subsistance ou chasse traditionnelle est celle exercée par les communautés locales sur leur territoire, en vue de satisfaire leurs besoins de consommation individuels et familiaux.

Par dérogation aux dispositions du présent chapitre, la chasse traditionnelle est exercée dans des conditions qui sont déterminées par arrêté du Ministre chargé de la faune.

ARTICLE 116 : Le droit de chasse est reconnu à toute personne âgée d'au moins 18 ans.

ARTICLE 117 : Les conditions d'utilisation des armes à feu dans le cadre de l'exercice de la chasse sont déterminées par arrêté du ministre chargé de la faune.

ARTICLE 118 : L'acte de chasse donne lieu à l'acquittement de taxes d'abattage, dans des conditions qui sont déterminées par arrêté conjoint des Ministres chargés de la faune et des finances.

ARTICLE 119 : Nul ne peut, sauf dérogation expresse de la loi, accomplir licitement un acte de chasse s'il n'est titulaire d'un permis de chasse.

ARTICLE 120 : Il est institué trois catégories de permis de chasse sportive :

- le permis de chasse national ou permis de catégorie A, délivré aux personnes de nationalité burkinabé ;

- le permis de chasse étranger résident ou permis de catégorie $B$, délivré aux personnes de nationalité étrangère résidant au Burkina Faso depuis au moins 6 mois ;

- le permis de chasse touristique ou permis de catégorie C, délivré aux personnes étrangères ne résidant pas au Burkina Faso.

ARTICLE 121 : Chaque catégorie de permis comporte trois degrés conférant respectivement le droit de pratiquer soit la petite chasse, soit la moyenne chasse, soit la grande chasse. Chaque degré de permis autorise l'abattage d'espèces déterminées qui sont précisées par arrêté du Ministre chargé de la faune. 
ARTICLE 122 : Lexercice de certaines activités particulières de chasse et de capture est subordonné à l'obtention de permis spéciaux : les permis de chasse et de capture scientifiques et les permis de capture commerciale.

ARTICLE 123 : Les permis de chasse et de capture scientifiques sont délivrés par le ministère chargé de la faune à des organismes scientifiques reconnus, pour les autoriser à abattre ou à capturer des animaux sauvages, y compris des espèces intégralement protégées, dans le seul but de satisfaire les nécessités de la recherche scientifique.

ARTICLE 124 : Les permis de capture commerciale sont délivrés à des personnes agréées pour les autoriser à capturer des animaux sauvages vivants, en vue de leur revente.

ARTICLE 125 : Tous les permis de chasse et de capture prévus dans la présente section sont délivrés par les services techniques chargés de la faune pour une ou plusieurs zones déterminées et pour une durée également déterminée.

\section{SECTION 2 : DE L'EXERCICE DE LA CHASSE}

ARTICLE 126 : La saison de chasse est déterminée chaque année par arrêté du Ministre chargé de la faune.

ARTICLE 127 : Exceptionnellement, pour les besoins de reconstitution de la faune, la chasse peut être temporairement interdite sur tout ou partie du territoire national.

La décision de fermeture de la chasse est prise par décret en Conseil des Ministres. Ce décret indique la durée de l'interdiction qui, dans tous les cas, ne peut excéder trois saisons de chasse.

ARTICLE 128 : Dans les réserves partielles de faune et les refuges locaux, l'exercice de la chasse est soumis aux conditions particulières d'accès prévues par les textes en vigueur.

ARTICLE 129: Sur les propriétés privées, l'exercice de la chasse appartient exclusivement au propriétaire, qui reste soumis à l'observation de la législation de la chasse.

Le propriétaire peut cependant autoriser toute autre personne à chasser sur ses terres.

ARTICLE 130 : Il est formellement interdit de chasser les animaux suités et les femelles gestantes.

ARTICLE 131 : Sont interdits sur l'ensemble du territoire national la chasse de nuit, la chasse à l'aide du feu, au moyen de produits chimiques ou toxiques, de pièges, d'éclairages aveuglants et, plus généralement, tous moyens de destruction massive ou sélective de la faune.

\section{SECTION 3 : DES GUIDES DE CHASSE}

ARTICLE 132 : Dans les réserves de faune et les refuges locaux, concédés ou non, la conduite des expéditions de chasse est exclusivement réservée à des professionnels agréés, appelés guides de chasse.

ARTICLE 133 : Le guide de chasse est une personne physique faisant profession de conduire des expéditions de chasse sportive au profit d'une clientèle. Dans l'exercice de son activité, le guide peut se faire assister par des pisteurs expérimentés.

ARTICLE 134 : Le titre de guide de chasse est conféré aux personnes âgées de 30 ans au moins et de 60 ans au plus, qui subissent avec succès les épreuves d'un examen organisé à cet effet.

64 Textes et textes de loi sur la gestion des ressources naturelles au Burkina Faso 
ARTICLE 135 : L'examen de guide de chasse vise à contrôler les con-naissances des guides en matière de réglementation faunique et de sécurité, d'identification des espèces, ainsi que leurs aptitudes en matière de maniement des armes.

Les modalités d'organisation de l'examen de guide de chasse sont déterminées par arrêté du Ministre chargé de la faune.

ARTICLE 136 : Le guide de chasse doit être détenteur d'une licence de guide de chasse délivrée par l'administration chargée de la faune, après paiement d'une redevance dont le montant est déterminé par arrêté conjoint des Ministres chargés de la faune et des finances.

ARTICLE 137 : Les guides de chasse sont chargés de veiller à la sécurité de leurs clients. Ils sont solidairement responsables des dommages causés par eux aux tiers. Ils sont considérés comme complices de la violation par leurs clients de la réglementation faunique en vigueur, sauf à eux de prouver qu'ils ont fait tout ce qui était en leur pouvoir pour empêcher l'accomplissement du délit.

\section{SECTION 4 : DES PRODUITS DE LA CHASSE}

ARTICLE 138 : Les produits de la chasse sont les trophées et les dépouilles. Le trophée est la partie identifiable non périssable d'un animal.

La dépouille est la partie périssable ou la carcasse d'un animal.

ARTICLE 139 : Il est formellement interdit d'abandonner les dépouilles de gibier sur les lieux de chasse. En cas d'abandon motivé, le chasseur est tenu, en vue d'éviter les gaspillages de viande, d'en informer rapidement les services techniques locaux chargés de la faune ou le village le plus proche.

ARTICLE 140: Les produits de la chasse ne peuvent circuler ou être stockés qu’accompagnés des pièces pouvant justifier leur détention.

Les conditions de détention, de cession et de circulation des animaux sauvages morts ou vifs et des trophées, ainsi que les règles relatives à leur importation et à leur exportation, sont déterminées par les textes d'application.

ARTICLE 141 : Le commerce de la viande sauvage est autorisé. Il est exercé par des commerçants et des restaurateurs agréés.

Les commerçants et les restaurateurs agréés ne doivent accepter que de la viande sauvage provenant d'abattages régulièrement effectués.

\section{CHAPITRE 2 : DU RANCHING ET DE L'ÉLEVAGE FAUNIQUE}

\section{SECTION 1 : DU RANCHING}

ARTICLE 142 : Au sens de la présente loi, le ranching est une activité de production et d'exploitation fauniques en milieu naturel ouvert, consistant en la réalisation d'aménagements spéciaux destinés à favoriser le développement des animaux sauvages et leur attachement à leur territoire naturel.

ARTICLE 143 : L'exercice du ranching par les personnes privées est soumis à autorisation du Ministre chargé de la faune.

Les textes d'application précisent les conditions dans lesquelles cette autorisation est délivrée, ainsi que les activités qui peuvent être combinées sans dommage avec l'exploitation du ranch. 
ARTICLE 144 : Le mouvement naturel et les migrations des animaux hors du territoire du ranch ne doivent pas être entravés par l'érection de clôtures ou d'autres obstacles matériels.

ARTICLE 145 : Les populations fauniques exploitées dans le ranch doivent faire l'objet d'une surveillance régulière par les services techniques locaux chargés de la faune ou par l'exploitant, en vue d'une gestion rationnelle des prélèvements.

\section{SECTION 2 : DE L'ÉLEVAGE FAUNIQUE}

ARTICLE 146 : Au sens de la présente loi, l'élevage faunique est une activité de production à but lucratif d'animaux sauvages, maintenus en état de captivité ou de semi-liberté, en vue de la commercialisation de la viande sauvage et des produits de la faune.

ARTICLE 147 : Lélevage des espèces fauniques peut être organisé par les personnes physiques ou morales de droit privé sur leurs terres privées.

ARTICLE 148 : L'élevage d'animaux sauvages est soumis à l'autorisation préalable du Ministre chargé de la faune.

ARTICLE 149 : Toutes les espèces de faune, intégralement ou partiellement protégées peuvent être élevées. Cependant, la capture et la vente des espèces intégralement protégées sont soumises aux conditions spécifiques précisées par les textes d'application.

L'exploitant est tenu de déclarer les espèces faisant l'objet de son élevage.

ARTICLE 150 : L'aire de l'élevage faunique doit être clôturée par tout moyen approprié.

ARTICLE 151 : Les animaux de l'élevage faunique sont la propriété de l'éleveur. Ils sont soustraits au régime général de la faune, notamment à celui de la chasse.

Le propriétaire de l'élevage faunique est responsable des dommages causés aux tiers par ses animaux sauvages.

ARTICLE 152 : La commercialisation des produits de l'élevage faunique est soumise, sauf dérogation, aux dispositions générales applicables en la matière faunique, ainsi qu'aux conventions internationales ratifiées par le Burkina Faso.

\section{CHAPITRE 3 : DES CONCESSIONS \\ SECTION 1 : DU PRINCIPE DE LA CONCESSION}

ARTICLE 153 : Le droit d'exploiter les ressources fauniques dans les réserves partielles de faune, les refuges locaux et les ranches de l'Etat peut être concédé à des personnes physiques ou morales de droit privé, en vue de l'organisation lucrative d'activités de chasse ou de ranching.

ARTICLE 154: La concession est toujours accordée à titre onéreux, moyennant le paiement d'une redevance annuelle fixée en fonction des potentialités de la zone concédée.

La concession confere à son bénéficiaire l'exclusivité de l'exploitation de la zone concédée.

ARTICLE 155 : La concession est accordée par le Ministre chargé de la faune par voie d'appel d'offres, pour une durée minimum de cinq ans. Les textes d'application de la présente loi précisent les conditions de l'appel d'offres.

ARTICLE 156 : Tout concessionnaire de droits d'exploitation faunique est tenu à l'observation stricte d'un cahier des charges, défini arrêté du Ministre chargé de la faune.

66 Textes et textes de loi sur la gestion des ressources naturelles au Burkina Faso 
ARTICLE 157 : Le concessionnaire a dans un délai maximum fixé par l'acte de concession, l'obligation d'élaborer un plan d'aménagement de la zone concédée et de le soumettre à l'approbation de l'administration territorialement compétente chargée de la faune.

\section{SECTION 2 : DES CONCESSIONS DE CHASSE ET DE TOURISME DE VISION}

ARTICLE 158: Leconcessionnaired'unezone doit être titulaire d'unelicenced'exploitation qui lui est délivrée par le Ministre chargé de la faune, et qui est renouvelée chaque année après paiement de la redevance annuelle. Il ne peut bénéficier de l'exploitation de plus d'une zone sur l'ensemble du territoire national.

ARTICLE 159 : La conduite des expéditions de chasse ou tourisme de vision dans les zones concédées relève de la compétence exclusive des guides de chasse ou tourisme de vision. Le concessionnaire est libre de négocier avec le guide de son choix.

ARTICLE 160 : Le cahier des charges des concessions de zone détermine les obligations du concessionnaire, notamment celles relatives à la mise en valeur, à l'exploitation effective de la zone et à la conservation des ressources fauniques. Il précise les infrastructures minimales à réaliser.

Le cahier des charges des concessionnaires de zone définit également les principes qui doivent gouverner les relations entre le concessionnaire et la population de la zone concernée.

\section{SECTION 3 : DES CONCESSIONS DE RANCH}

ARTICLE 161 : Ne peuvent bénéficier d'une concession de ranch que les personnes munies d'une autorisation du Ministre chargé de la faune. L'autorisation est renouvelée chaque année après paiement effectif de la redevance annuelle.

Nul ne peut bénéficier de plus d'une concession de ranch sur l'ensemble du territoire national.

ARTICLE 162 : Le cahier des charges des concessionnaires de ranch précise les obligations du concessionnaire, notamment celles relatives à l'aménagement, à la mise en valeur et à la conservation des ressources fauniques. Il précise également les activités pouvant être utilement combinées avec le ranching.

ARTICLE 163 : Le concessionnaire d'un ranch peut vendre des droits d'exploitation à un promoteur d'activités touristiques en vue notamment de l'organisation de circuits touristiques de vision.

\section{LIVRE III - DE LA PECHE ET DE LAQUACULTURE}

\section{TITRE I - DE LA REGLEMENTATION GENERALE ET DES PRINCIPES DE CONSERVATION DES RESSOURCES}

\section{CHAPITRE 1 : DES DÉFINITIONS}

ARTICLE 164 : Les dispositions du présent livre s'appliquent à l'ensemble des eaux domaniales, telles que définies par les textes en vigueur notamment ceux de la RAF.

Elles ne s'appliquent pas sauf dispositions expresses aux eaux privées, c'est-à-dire aux eaux d'étangs, mares ou fossés creusés sur des fonds privés dans lesquels les poissons qui vivent en eau libre ne peuvent pénétrer naturellement. 
ARTICLE 165 : Au sens de la présente loi, la pêche consiste dans les actes tendant à la capture ou à l'extraction, par tout moyen autorisé et pour toute utilisation que ce soit, des ressources halieutiques contenues dans les eaux burkinabé.

ARTICLE 166 : Au sens de la présente loi, l'aquaculture est l'élevage d'organismes aquatiques, y compris notamment les poissons, les mollusques, les crustacés, les batraciens et les végétaux.

ARTICLE 167 : On entend par ressource halieutique tout organisme vivant dans l'eau et pouvant en être retiré.

ARTICLE 168 : Au sens de la présente loi, l'unité de pêche désigne le groupe composé d'un maître pêcheur et de ses aides. Une unité de pêche ne peut comprendre plus de deux aides.

\section{CHAPITRE 2 : DE LA CONSERVATION DES RESSOURCES HALIEUTIQUES}

ARTICLE 169 : Sans préjudice des dispositions réglementaires plus contraignantes, il est interdit pour exercer la pêche :

a) d'utiliser des substances toxiques destinées à étourdir affaiblir ou tuer tout organisme aquatique ;

b) de se servir d'explosifs ou d'armes à feu ;

c) de faire usage de procédés électromagnétiques ou d'électrocution de tout organisme aquatique ;

d) d'utiliser des engins confectionnés avec de l'étoffe ou du grillage moustiquaire permettant la capture d'alevins.

ARTICLE 170 : La pêche dans les frayères ainsi que la destruction du frais et des alevins sont interdites de manière permanente.

Après consultation des autres administrations compétentes, un arrêté du Ministre chargé des pêches et de l'aquaculture précise les délimitations géographiques des frayères dans lesquelles la pêche est interdite. Cet arrêté peut être complété le cas échéant, par une réglementation des autorités locales.

ARTICLE 171 : Une autorisation du Ministre chargé des pêches et de l'aquaculture est exigée pour l'introduction dans les eaux burkinabé, des espèces ou des oeufs de poissons en provenance de l'étranger. La présente disposition s'applique également aux eaux privées telles que définies au chapitre premier, Titre I du présent livre.

Un arrêté ministériel dresse la liste des poissons se trouvant déjà dans les eaux burkinabé et dont la manipulation et le transfert d'une région à une autre à l'intérieur du pays ne nécessite aucune autorisation préalable.

ARTICLE 172 : Le ministère chargé des pêches et de l'aquaculture encourage et favorise l'empoissonnement, le réempoissonnement et l'alevinage des eaux burkinabé. Ces activités sont soumises à autorisation préalable des services techniques compétents.

\section{CHAPITRE 3 : DES POUVOIRS RÉGLEMENTAIRES}

ARTICLE 173 : Le Ministre chargé des pêches et de l'aquaculture peut fixer pour l'ensemble des eaux burkinabé ou pour une partie seulement

a) les périodes, saisons, heures pendant lesquelles la pêche de toutes ou certaines espèces est interdite ; 
b) les zones où la pêche est interdite à titre temporaire ou de manière permanente ;

c) les zones ou les périodes pour lesquelles, l'utilisation de certaines techniques est interdite ;

d) les dimensions en-dessous desquelles la capture de certaines espèces est interdite;

e) les caractéristiques des embarcations, engins et instruments de pêchedontl'usage est autorisé, les dimensions minimales des mailles des filets et la façon de les mesurer;

f) les substances et procédés de pêche prohibés ;

g) toute autre mesure visant à protéger et à conserver les ressources halieutiques.

ARTICLE 174 : Le Ministre chargé des pêches et de l'aquaculture peut fixer sur tout ou partie du territoire burkinabé :

a) des mesures de réglementation relatives à l'importation, à la détention, au transport, à la vente et à l'achat d'engins de pêche, d'embarcations, de substances et de matières interdites par la législation en vigueur ;

b) des mesures de réglementation portant sur la détention, le transport, la vente et l'achat de poisson pêché par des moyens interdits ou dont la pêche est prohibée ;

c) toute autre mesure visant à rendre la législation en matière de pêche plus efficace.

ARTICLE 175 : Dans les limites de leurs compétences tant administratives que territoriales, les autorités locales peuvent en cas d'urgence, prendre une ou plusieurs des mesures définies dans le présent chapitre. Ces mesures doivent être communiquées dans les plus brefs délais au Ministre chargé des pêches et de l'aquaculture.

ARTICLE 176 : Dans les limites des périmètres aquacoles d'intérêt économique, le responsable du comité de gestion dispose de prérogatives réglementaires déléguées par le Ministre chargé des pêches et de l'aquaculture. La composition et la nature de celles-ci sont définies par arrêté ministériel.

\section{CHAPITRE 4 : DES PLANS DE GESTION ET D'AMÉNAGEMENT DES ACTIVITÉS DE PÊCHE ET D'AQUACULTURE}

ARTICLE 177 : Le ministère chargé des pêches et de l'aquaculture établit, développe et renouvelle périodiquement un plan de gestion et d'aménagement des activités de pêche et d'aquaculture.

ARTICLE 178 : Ce plan doit notamment procéder à une analyse de l'état de la pêche et de l'aquaculture et évaluer les ressources halieutiques afin de déterminer l'effort de pêche pouvant être entrepris dans les eaux burkinabé.

Il doit exposer les objectifs à atteindre à court et moyen termes, définir les mesures de gestion et d'aménagement nécessaires à la réalisation des objectifs pré-cités et prévoir toutes mesures tendant à promouvoir la pêche professionnelle et la consommation de poisson au sein de la population burkinabé.

Le plan a un caractère général, mais peut inclure des mesures spécifiques aux différentes régions.

ARTICLE 179 : Dans le cadre de la préparation ou de la révision du plan, le ministère chargé des pêches et de l'aquaculture organise la consultation de l'ensemble des partenaires administratifs, économiques, scientifiques et sociaux. 


\section{TITRE II - DE LA GESTION ET DU DEVELOPPEMENT DES ACTIVITES DE LA PECHE}

\section{CHAPITRE 1 : DES TYPES DE PÊCHE ET DE L'ORGANISATION}

ARTICLE 180 : En fonction de sa finalité, la pêche peut être commerciale, de subsistance, sportive ou scientifique.

ARTICLE 181 : La pêche commerciale est pratiquée dans un but lucratif et donne lieu à la vente de tout ou partie des captures.

La pêche de subsistance a pour but fondamental la capture de ressources halieutiques destinées à la consommation du pêcheur et de sa famille et ne donne pas lieu à leur vente.

La pêche sportive est pratiquée sans but lucratif à des fins récréatives.

La pêche scientifique a pour but l'étude et la connaissance des ressources halieutiques.

ARTICLE 182 : Dans les eaux publiques naturelles ou artificielles, les activités de pêche sont organisées par l'Etat.

Dans les eaux privées, les activités de pêche sont organisées par le propriétaire.

ARTICLE 183 : La pêche dans les eaux burkinabé est réservée aux seuls nationaux. Cependant l'exercice de la pêche peut être accordé aux ressortissants des autres Etats sous réserve de réciprocité.

\section{CHAPITRE 2 : DES AUTORISATIONS DE PÊCHE SECTION 1 : DES DISPOSITIONS GÉNÉRALES}

ARTICLE 184 : L'exercice des différents types de pêche est soumis à l'obtention d'un permis de pêche sauf exception prévue par la loi.

ARTICLE 185 : Toute personne désirant se livrer aux activités de pêche sportive ou commerciale au Burkina Faso doit posséder le permis de pêche spécifique à la région dont relève le lieu d'exercice de son activité. Ce permis de pêche ne peut être utilisé que dans la région pour laquelle il a été délivré.

ARTICLE 186 : Au sein d'une unité de pêche, chaque membre doit posséder à titre individuel un permis de pêche. Seul le maître de pêche est habilité à effectuer une demande de permis de pêche, tant pour son usage personnel que pour celui de ses aides.

ARTICLE 187 : Les autorités compétentes pour la délivrance des permis de pêche sont définies par arrêté ministériel, sauf pour ce qui concerne le régime de gestion spéciale défini à l'article 210 du présent code.

ARTICLE 188 : La délivrance du permis de pêche est assujettie à l'acquittement d'une redevance dont le montant et les modalités de paiement sont fixés par arrêté ministériel.

ARTICLE 189 : La durée du permis de pêche ainsi que les critères et conditions de son attribution sont définis par arrêté ministériel.

ARTICLE 190 : Les permis de pêche sont strictement personnels et ne peuvent être ni prêtés ni vendus.

ARTICLE 191 : La délivrance ou le renouvellement du permis est refusé lorsque le demandeur ne remplit pas les conditions définies par arrêté ministériel.

\begin{tabular}{l|l}
70 & Textes et textes de loi sur la gestion des ressources naturelles au Burkina Faso
\end{tabular} 
ARTICLE 192 : En outre le permis de pêche peut être refusé, suspendu ou révoqué :

a) afin d'assurer la conservation des ressources halieutiques, de garantir une gestion adéquate de ces mêmes ressources ou d'exécuter les objectifs de gestion et d'aménagement des pêcheries ;

b) si le demandeur du permis a été reconnu coupable de récidive en matière d'infraction aux dispositions de la présente loi ou de tout autre texte réglementaire relatif à la pêche et à l'aquaculture dans les douze mois qui précèdent la demande.

ARTICLE 193 : Un permis de pêche ne peut être révoqué ou suspendu par les autorités compétentes que pour l'un des motifs visés à l'article 192 ci-dessus.

ARTICLE 194 : Le refus de délivrance, le non renouvellement, la suspension ou la révocation du permis de pêche doivent toujours être motivés.

ARTICLE 195 : Les permis de pêche sont établis dans les formes prescrites par arrêté ministériel et leur titulaire est soumis au respect des conditions définies par la présente loi ou par les textes réglementaires pris pour son application.

Le Ministre chargé des pêches et de l'aquaculture peut assortir la délivrance du permis de conditions spéciales jugées importantes, telles que celles relatives au type et caractéristiques des embarcations, aux zones à l'intérieur desquelles la pêche est autorisée, aux espèces et quantités de poissons dont la capture est permise ainsi qu'aux obligations statistiques.

\section{SECTION 2 : DES DISPOSITIONS SPÉCIFIQUES}

ARTICLE 196 : Les personnes se livrant à des opérations de pêche dans un but scientifique sont dispensées de l'obligation de détenir un permis de pêche.

ARTICLE 197 : La réalisation d'opérations de pêche à but scientifique dans les eaux burkinabé est soumise à autorisation écrite préalable du Ministre chargé des pêches et de l'aquaculture. Les conditions de délivrance de l'autorisation ainsi que les limitations éventuelles imposées sont définies par arrêté du Ministre chargé des pêches et de l'aquaculture.

ARTICLE 198 : Les personnes se livrant à des opérations de pêche à des fins de subsistance sont libérées de l'obligation de détenir un permis de pêche.

La pêche coutumière annuelle est libre et gratuite et est organisée par les autorités villageoises traditionnelles sous le contrôle des services techniques chargés de la pêche.

ARTICLE 199 : Dans les aires soumises à un régime de protection spéciale tels les parcs nationaux et les forêts classées, les activités de pêche s'exercent conformément aux dispositions particulières y afférent.

\section{CHAPITRE 3 : DES CONCESSIONS DE PÊCHE}

\section{SECTION 1 : DE LA DÉFINITION ET DU RÉGIME}

ARTICLE 200 : La concession de pêche est le procédé par lequel l'Etat confie à une personne physique ou morale de droit privé, la jouissance exclusive de tout ou partie d'un plan d'eau aux fins d'exploitation des ressources halieutiques qui y sont contenues.

ARTICLE 201 : La concession est accordée sous la forme d'un contrat à titre onéreux. La durée, les formes et les conditions de la concession sont déterminées par arrêté ministériel. 
ARTICLE 202 : Un cahier des charges arrêté lors de la conclusion du contrat fixe les droits et obligations des contractants. Le concessionnaire est tenu d'exercer les droits et les obligations à titre personnel.

\section{SECTION 2 : DE LATTRIBUTION}

ARTICLE 203 : Toute personne privée, physique ou morale, notamment les groupements de pêcheurs, peut introduire une demande de concession auprès des services du ministère chargé des pêches et de l'aquaculture.

Les conditions de la demande et de son instruction sont précisées par arrêté du Ministre chargé des pêches et de l'aquaculture.

ARTICLE 204 : Le Ministre chargé des pêches et de l'aquaculture peut décider d'attribuer une concession par la procédure d'appel d'offres. Les conditions d'une telle procédure sont définies par voie réglementaire.

ARTICLE 205 : La concession peut être modifiée ou résiliée pour les raisons suivantes :

a) désaccord entre les contractants ;

b) cause d'utilité publique ;

c) inexécution après mise en demeure d'une des conditions prévues au contrat ;

d) défaut de mise en valeur du plan d'eau concédé dans un délai de deux ans à compter de la signature du contrat ou de l'interruption de l'exploitation pendant une durée supérieure à six (6) mois, sans que le concessionnaire puisse invoquer la force majeure ou le cas fortuit;

e) violation des dispositions législatives ou réglementaires en vigueur.

ARTICLE 206 : Dans le cas où le contrat est modifié ou résilié pour cause d'utilité publique, le concessionnaire peut demander soit une autre concession de pêche de superficie équivalente et à des conditions similaires, soit une autre concession faisant l'objet d'un nouveau contrat.

Si l'obtention d'une autre concession n'est pas possible, et si la modification ou la résiliation entraine un dommage pour le concessionnaire, il y a alors lieu de procéder à l'indemnisation du préjudice subi par celui-ci. En cas de désaccord le litige sera soumis à la juridiction compétente.

ARTICLE 207 : La concession peut être renouvelée au profit de son titulaire. Le renouvellement peut donner lieu à la renégociation des termes du contrat à la demande de l'un des contractants.

ARTICLE 208 : La concession ne peut être ni modifiée ni transférée à un autre titulaire sans une autorisation écrite du Ministre chargé des pêches et de l'aquaculture.

ARTICLE 209: Outre le respect des droits et obligations inscrits au contrat de concession, les co-contractants sont soumis aux dispositions de la législation en matière de pêche et d'aquaculture.

\section{CHAPITRE 4 : DU RÉGIME DE GESTION SPÉCIALE}

\section{SECTION 1 : DES PLANS D'EAU CONCERNÉS ET DE LEUR RÉGIME}

ARTICLE 210 : Peuvent bénéficier d'un régime de gestion spéciale, les plans d'eau d'une superficie supérieure à 5.000 hectares en période d'étiage ayant une importance 
économique significative en matière de pêche et dont les ressources halieutiques sont menacées de surexploitation. Ces plans d'eau constituent des périmètres aquacoles d'intérêt économique.

ARTICLE 211 : La liste des plans d'eau entrant dans la catégorie visée ci-dessus est déterminée par décret en conseil des ministres sur proposition du Ministre chargé des pêches et de l'aquaculture.

ARTICLE 212 : L'exercice des activités de pêche sur les périmètres aquacoles d'intérêt économique est soumis :

a) à l'obtention d'un titre de pêche propre au périmètre aquacole d'intérêt économique concerné ;

b) au respect du nombre total de pêcheurs autorisés à exercer leurs activités sur le périmètre aquacole d'intérêt économique.

ARTICLE 213: Les titres de pêche propres aux périmètres aquacoles d'intérêt économique sont soumis au régime général des permis de pêche tel que défini dans le présent livre, à l'exception de la demande de ces permis qui est effectuée auprès du comité assurant la gestion du périmètre aquacole d'intérêt économique. Leur validité est circonscrite à l'étendue du périmètre aquacole d'intérêt économique visé.

\section{SECTION 2 : DE LA GESTION}

ARTICLE 214 : La gestion des ressources halieutiques et l'aménagement des pêcheries sur les périmètres aquacoles d'intérêt économique sont confiés à un comité de gestion créé pour chaque périmètre, et ci-après dénommé le comité.

ARTICLE 215 : Le comité a pour mission notamment de formuler le plan de gestion du périmètre aquacole d'intérêt économique et de délivrer les permis de pêche propres au périmètre.

La création, la composition, les missions et le fonctionnement du comité sont précisés par arrêté du ministre chargé des pêches et de l'aquaculture.

\section{TITRE III - DE LA GESTION ET DU DEVELOPPEMENT DES ACTIVITES D'AQUACULTURE}

\section{CHAPITRE 1 : DE LA DÉFINITION ET DE LA RÉGLEMENTATION}

ARTICLE 216 : On entend par établissement d'aquaculture, toutes exploitations d'élevage d'organismes aquatiques à des fins commerciales, scientifiques, expérimentales, de consommation ou de repeuplement.

ARTICLE 217 : Sans préjudice des règles domaniales pertinentes et du respect des compétences des autres ministères, le ministère chargé des pêches et de l'aquaculture peut prendre toute mesure tendant à réglementer les établissements d'aquaculture et à définir des critères d'attribution pour les demandes d'autorisation.

ARTICLE 218 : Nul ne peut créer un établissement d'aquaculture sans y être dûment autorisé par le Ministre chargé des pêches et de l'aquaculture.

Toute personne physique ou morale de droit public ou privé possédant les connaissances et compétences techniques et les moyens économiques nécessaires au développement des activités d'aquaculture est qualifiée pour demander une autorisation d'aquaculture. Les critères liés à la qualité de demandeur sont définis par arrêté ministériel. 
ARTICLE 219 : La création d'un établissement d'aquaculture ne peut être accordée si un inconvénient paraît devoir en résulter pour le peuplement piscicole des eaux avec lesquelles cette aquaculture communiquerait, et notamment lorsque sa création aurait pour conséquence l'interruption :

- l'interruption de la libre circulation des espèces piscicoles dans les cours d'eau ;

- l'insuffisance du débit ou l'altération de la qualité de l'eau compromettant la vie de ces espèces.

ARTICLE 220 : Tout établissement d'aquaculture doit posséder un dispositif permettant de couper toute communication entre ses propres eaux et les eaux du domaine public. En cas de pollution des eaux ou de maladie affectant les poissons de l'établissement d'aquaculture, le Ministre chargé des pêches et de l'aquaculture peut ordonner la mise en oeuvre de mesures de sauvegarde additionnelle.

ARTICLE 221 : La demande d'autorisation pour la création d'un établissement d'aquaculture à des fins scientifiques ou expérimentales doit être accompagnée d'un plan détaillé du projet à réaliser. Le Ministre chargé des pêches et de l'aquaculture peut exiger :

a) que des scientifiques burkinabé qu’il aura désigné soient associés aux opérations, aux frais du bénéficiaire de l'autorisation ;

b) que la totalité des données recueillies au cours des opérations de recherche ainsi que les résultats obtenus après traitement et analyse soient communiqués au Ministère chargé des pêches et de l'aquaculture.

ARTICLE 222 : L'autorisation de création d'un établissement d'aquaculture est accordée par le Ministre chargé des pêches et de l'aquaculture après consultation des Ministres chargés de l'eau, de l'agriculture, des ressources animales et des domaines.

\section{CHAPITRE 2 : DES CONCESSIONS D'AQUACULTURE}

ARTICLE 223 : Toute personne physique ou morale de droit public ou privé qui veut aménager à des fins d'aquaculture, des terres ou eaux faisant partie du domaine public ou des terres nécessitant le prélèvement d'eaux provenant du domaine public est tenue de demander une concession à l'Etat.

Les formes, conditions et durée du contrat de concession sont définies par les textes d'application de la présente loi.

ARTICLE 224 : Un cahier des charges arrêté lors de la conclusion du contrât fixe les droits et obligations des co-contractants. Le concessionnaire est tenu d'exercer les droits et les obligations à titre personnel.

ARTICLE 225 : La demande de concession est examinée par le ministère chargé des pêches et de l'aquaculture qui peut, s'il le juge nécessaire ou sur la demande du Ministre chargé de l'environnement, faire procéder à une étude d'impact sur l'environnement. Si tel est le cas et si les résultats de celle-ci sont fortement défavorables, alors la concession d'aquaculture ne peut être octroyée.

ARTICLE 226 : La concession est accordée par le Ministre chargé des pêches et de l'aquaculture après avis des Ministres chargés de l'eau, de l'agriculture, des ressources animales et des domaines.

Tout refus d'une concession d'aquaculture doit être motivé.

74 Textes et textes de loi sur la gestion des ressources naturelles au Burkina Faso 
ARTICLE 227 : Les dispositions relatives au renouvellement et au transfert des concessions de pêche sont applicables dans les mêmes termes aux concessions d'aquaculture.

\section{TITRE IV - DE LA PROTECTION DES EAUX, DE LHYGIENE ET DE LA QUALITE DES PRODUITS HALIEUTIQUES}

\section{CHAPITRE 1 : DES MESURES PARTICULIÈRES DE PROTECTION DES EAUX}

ARTICLE 228 : Préalablement à l'octroi de toute autorisation relative à l'occupation, à l'aménagement ou à la dénudation des berges des plans d'eau, et dans le cas où les intérêts de la pêche ou de l'aquaculture sont susceptibles d'être affectés, l'administration compétente doit consulter le ministère chargé des pêches et de l'aquaculture.

ARTICLE 229: Lorsqu'ils sont de nature à détruire les frayères, les zones de croissance ou les zones d'alimentation ou de réserve de la nourriture de la faune piscicole, l'installation ou l'aménagement d'ouvrages, ainsi que l'exécution de travaux dans le lit du cours d'eau sont soumis à l'avis préalable du ministère chargé des pêches et de l'aquaculture.

ARTICLE 230 : Avant d'autoriser ou d'entamer des opérations de dérivation, captage, pompage direct ou indirect d'eau susceptible de modifier les débits ou d'entraver la circulation des organismes aquatiques, et plus généralement tous travaux susceptibles d'affecter les intérêts de la pêche et de l'aquaculture, le Ministre chargé de l'environnement doit faire procéder à une étude d'impact sur l'environnement et doit recueillir l'avis préalable du Ministre chargé des pêches et de l'aquaculture.

ARTICLE 231 : Tout rejet ou déversement de substances dans l'eau est exécuté conformément aux dispositions du code de l'environnement et dans le respect des normes édictées par arrêté conjoint des Ministres chargés de l'eau, de la santé, de l'environnement et de l'industrie.

ARTICLE 232 : Toute personne ayant jeté, déversé, ou laissé écouler dans les cours d'eau, directement ou indirectement, des substances quelconques dont l'action ou la réaction a détruit le poisson ou nui à sa nutrition, à sa reproduction, à son habitat ou à sa valeur alimentaire est sanctionnée conformément aux dispositions des textes sur l'eau.

\section{CHAPITRE 2 : DES MESURES D'HYGIÈNE ET DE CONTRÔLE SANITAIRE DES PRODUITS}

ARTICLE 233 : En accord avec les autres Ministres compétents, notamment ceux chargés de la santé et des ressources animales, le Ministre chargé des pêches et de l'aquaculture fixe les normes d'hygiène et de qualité des produits de la pêche et de l'aquaculture.

ARTICLE 234 : En étroite collaboration avec les Ministres chargés de la santé, et des ressources animales, le Ministre chargé des pêches et de l'aquaculture doit promouvoir l'adoption et l'application de mesures de contrôle sanitaire des captures effectuées dans les eaux burkinabè.

ARTICLE 235: Au sens du présent code, on entend par établissement de traitement de poisson, tout local ou installation dans lequel le poisson est mis en boîte, séché, mis en saumure, salé, fumé ou réfrigéré, mis en glace ou congelé ou traité de toute autre manière pour être vendu au Burkina Faso ou à l'étranger.

ARTICLE 236 : Sans préjudice des attributions propres aux autres ministères compétents, le choix du site, la constitution et le fonctionnement d'établissements de traitement 
de poisson sont soumis à l'autorisation préalable du ministère chargé des pêches et de l'aquaculture.

ARTICLE 237 : Le ministère chargé des pêches et de l'aquaculture détermine, en accord avec les autres ministères compétents, les normes d'hygiène et de qualité relatives à la construction, au fonctionnement et à la production des établissements de traitement de poisson et aux conditions de contrôle des activités desdits établissements.

\section{CHAPITRE 3 : DU TRANSPORT ET DE LA COMMERCIALISATION DES PRODUITS HALIEUTIQUES}

Article 238 : Le Ministre chargé des pêches et de l'aquaculture prend, en collaboration avec les autres Ministres compétents, les mesures appropriées relatives aux conditions de transport des produits de la pêche et de l'aquaculture.

Article 239 : Le Ministre chargé du commerce en collaboration avec le Ministre chargé des pêches et de l'aquaculture élabore et soumet au Conseil des Ministres, les projets législatifs et réglementaires nécessaires à l'organisation rationnelle des circuits de commercialisation et de distribution des produits halieutiques.

Article 240 : Nul ne peut se livrer au commerce des produits halieutiques sans être titulaire d'une licence de commercialisation de ce type de produits.

\section{LIVRE IV - DE LA REPRESSION DES INFRACTIONS}

\section{TITRE I - DES PROCEDURES}

\section{CHAPITRE 1 : DE LA RECHERCHE ET DE LA CONSTATATION DES INFRACTIONS}

ARTICLE 241 : Les infractions aux dispositions du présent code sont recherchées et constatées par des agents assermentés des Eaux et Forêts et des services partenaires et par les officiers de police judiciaire.

ARTICLE 242 : Les agents assermentés peuvent pénétrer dans les maisons en vue de procéder à des enquêtes, constats et perquisitions. Le droit de perquisition doit être exercé par au moins deux agents, ou un agent et au moins deux témoins.

Toutefois la perquisition reste interdite de 21 h 00 à 6 h 00 hors les cas de flagrant délit.

ARTICLE 243 : Les agents assermentés peuvent visiter tout véhicule ou embarcation chaque fois que le service l'exige. En cas de besoin, ils peuvent se faire assister dans leur mission d'un représentant de la force publique.

ARTICLE 244 : Les constats, enquêtes et perquisitions font l'objet de procès-verbaux établis par les agents assermentés des eaux et forêts ou par les officiers de police judiciaire.

Le procès-verbal contient l'exposé précis des faits, de toutes les circonstances pertinentes ainsi que les identités et déclarations des témoins éventuels.

ARTICLE 245 : Les procès-verbaux dressés par les agents assermentés font foi jusqu’à inscription de faux.

Le prévenu qui veut s'inscrire en faux contre un procès-verbal est tenu de le faire au moins huit (8) jours avant l'audience indiquée par la citation.

ARTICLE 246 : Les agents non assermentés des eaux et forêts ne peuvent établir que des rapports, ceux-ci font foi seulement jusqu’à preuve contraire.

76 $\mid$ Textes et textes de loi sur la gestion des ressources naturelles au Burkina Faso 


\section{CHAPITRE 2 : DES ACTIONS ET DES POURSUITES}

ARTICLE 247 : Les actions et poursuites devant les juridictions pénales compétentes sont exercées directement par le directeur général de l'administration des Eaux et Forêts ou son représentant, sans préjudice du droit qui appartient au ministère public près ces juridictions.

Le directeur général de l'administration des Eaux et Forêts ou son représentant a le droit d'exposer l'affaire devant le tribunal et de déposer ses conclusions.

ARTICLE 248 : Les jugements en matière forestière, de faune et de pêche sont signifiés au directeur général de l'administration forestière qui peut interjeter appel des jugements rendus en premier ressort dans les délais prévus par le Code de procédure pénale.

ARTICLE 249 : Laction publique en matière d'infraction à la législation forestière, faunique et de pêche se prescrit par un an.

\section{CHAPITRE 3 : DES SAISIES ET DES CONFISCATIONS}

ARTICLE 250 : Tout produit forestier, de faune ou de pêche récolté de manière frauduleuse est confisqué sans préjudice de l'application des sanctions pénales applicables. Les moyens qui ont servi à commettre l'infraction sont saisis à titre de mesure conservatoire jusqu'à règlement du différend.

ARTICLE 251 : En cas de confiscation de produits forestiers, fauniques ou de pêche, le procès verbal de constatation des infractions porte mention desdites confiscations.

ARTICLE 252: Les produits forestiers, fauniques et de la pêche provenant des confiscations sont vendus par voie d'adjudication publique par le directeur général de l'administration des Eaux et Forêts.

\section{CHAPITRE 4 : DES TRANSACTIONS}

ARTICLE 253 : Avant et pendant le jugement, les poursuites relatives à la législation forestière, faunique et de la pêche peuvent être arrêtées moyennant l'acceptation par le délinquant d'une transaction, proposée par le Directeur Général de l'Administration des Eaux et Forêts ou ses représentants habilités à cet effet.

ARTICLE 254 : Le montant des transactions doit être obligatoirement acquitté dans les délais fixés dans l'acte de transaction, faute de quoi, il est procédé aux poursuites judiciaires. Trente pour cent $(30 \%)$ des amendes et transaction sont versés aux verbalisateurs et indicateurs. Les textes d'application en fixent les modalités de prélèvement et de répartition.

\section{TITRE II - DES DIFFERENTES INFRACTIONS ET PENALITES}

ARTICLE 255 : Sans préjudice des confiscations, restitutions ou remises en état qui pourraient être prononcées par les tribunaux, les infractions à la législation forestière, faunique et de la pêche sont punies des sanctions prévues aux articles 258 et suivants.

ARTICLE 256: Ces peines sont portées au double en cas de récidive, d'infraction commise de nuit ou dans une aire de protection de la faune. Il en est de même en cas d'infraction commise par un concessionnaire, un guide ou un agent des services des Eaux et Forêts.

ARTICLE 257 : Les dispositions du code de procédure pénale relatives au crime ou délit de rébellion sont applicables à toute attaque, toute résistance avec violence et voies de fait envers les agents des Eaux et Forêts agissant pour l'exécution des règlements, des ordres ou ordonnances de l'autorité publique. 


\section{CHAPITRE 1 : DES INFRACTIONS EN MATIÈRE FORESTIÈRE}

ARTICLE 258 : Sont punis d'une amende de cent mille francs à un million de francs (100.000 F à 1.000.000 F) et d'un emprisonnement de six (6) mois à trois (3) ans ou de l'une de ces deux peines seulement :

- ceux qui allument intentionnellement des feux incontrôlés dans les forêts classées, parcs nationaux et autres aires protégées ;

- ceux qui procèdent à la destruction d'un périmètre de reboisement ou de restauration; ceux qui réalisent des activités d'exploitation industrielle des produits forestiers sans autorisation préalable.

ARTICLE 259 : Sont punis d'une amende de cinquante mille francs à cinq cent mille francs (50.000 F à 500.000 F), et d'un emprisonnement de trois (3) mois à deux (2) ans ou de l'une de ces deux peines seulement :

- ceux qui allument des feux incontrôlés sur le domaine forestier protégé ;

- ceux qui par négligence ou imprudence provoquent des feux incontrôlés dans les forêts classées ;

- ceux qui procèdent à des défrichements non autorisés dans les forêts classées ;

- ceux qui procèdent à la destruction d'essences forestières protégées.

ARTICLE 260 : Sont punis d'une amende de vingt mille francs à deux cent mille francs (20.000 F à 200.000 F) et d'un emprisonnement d'un mois (1) à un an (1) ou de l'une de ces deux peines seulement :

- ceux qui réalisent des activités d'exploitation commerciale des produits forestiers sans autorisation préalable ;

- ceux qui laissent divaguer les animaux dans les forêts non ouvertes à leur pâturage ;

- ceux qui procèdent au stockage ou au transport de produits forestiers sans autorisation;

- ceux qui procèdent à la coupe de bois vert sans autorisation.

ARTICLE 261 : Sont punis d'une amende de cinq mille francs à cinquante mille francs $(5.000 \mathrm{~F}$ à $50.000 \mathrm{~F})$ :

- ceux qui procèdent à l'ébranchage ou à la mutilation des arbres ;

- ceux qui circulent dans une forêt classée à des fins touristiques, scientifiques ou autres, sans autorisation ;

- ceux qui procèdent au déplacement ou à la destruction des bornes, balises et autres moyens de délimitation du domaine forestier classé.

ARTICLE 262 : Dans un but d'éducation, et en tenant compte des circonstances de l'espèce, le tribunal peut remplacer les sanctions prévues à l'article précédent par un travail d'intérêt commun.

\section{CHAPITRE 2 : DES INFRACTIONS EN MATIÈRE DE FAUNE}

ARTICLE 263 : Sont passibles d'une amende de cent mille francs à un million de francs $(100.000 \mathrm{~F}$ à 1.000.000 F) et d'un emprisonnement de six (6) mois à trois (3) ans ou de l'une de ces deux peines seulement :

78 Textes et textes de loi sur la gestion des ressources naturelles au Burkina Faso 
- ceux qui ont accompli des actes de chasse ou de capture à l'intérieur des parcs nationaux ou des réserves totales de faune ;

- ceux qui ont accompli des actes de chasse sur des espèces intégralement protégées.

ARTICLE 264 : Sont passibles d'une amende de cent mille francs à cinq cent mille francs (100.000 F à $500.000 \mathrm{~F}$ ) et d'un emprisonnement de trois (3) mois à deux (2) ans ou de l'une de ces deux peines seulement :

- ceux qui exercent la profession de concessionnaire ou de guide sans être détenteur d'une licence ;

- ceux qui organisent des activités cynégétiques en dehors des zones qui leur sont concédées.

ARTICLE 265 : Sont passibles d'une amende de cinquante mille francs à trois cent mille francs (50.000 F à $300.000 \mathrm{~F}$ ) et d'un emprisonnement de trois (3) mois à un (1) an ou de l'une de ces deux peines seulement :

- les personnes qui se livrent à la chasse sans permis de chasse, qui chassent en dehors des périodes légales d'ouverture de la chasse ou qui chassent au-delà des droits que leur confere leur permis;

- les personnes qui abattent des animaux suitées ou des femelles gestantes, ramassent les œufs ou détruisent les nids d'oiseaux ;

- les personnes qui se livrent à des actes de destruction gratuite de la faune, même sur des espèces qui ne sont inscrites sur aucune liste de protection.

ARTICLE 266. Sont passibles d'une amende de cinq mille francs à cinquante mille francs $(5.000 \mathrm{~F}$ à $50.000 \mathrm{~F})$ :

- les personnes qui entrent, circulent ou séjournent sans autorisation à l'intérieur des parcs nationaux et des réserves de faune ;

- les personnes qui commettent des actes prohibés ou se livrent à des activités interdites à l'intérieur des parcs nationaux et des réserves de faune ;

- les commerçants qui reçoivent et commercialisent de la viande sauvage qu'ils savent provenir d'activités illicites de chasse. Dans ce cas, le Ministre chargé de la faune peut ordonner la fermeture administrative de trois (3) mois à six (6) mois s'il s'agit d'un bar, d'un hôtel ou d'un restaurant ;

- les personnes qui détiennent ou élèvent des animaux sauvages sans autorisation.

ARTICLE 267 : Dans un but d'éducation, et en tenant compte des circonstances de l'espèce, le tribunal peut remplacer les sanctions prévues à l'article précédent par un travail d'intérêt commun.

\section{CHAPITRE 3 : DES INFRACTIONS EN MATIÈRE DE PÊCHE ET D'AQUACULTURE}

ARTICLE 268 : Sont passibles d'une amende de vint mille francs à deux cent mille francs (20.000 F à 200.000 F) et d'un emprisonnement de un (1) mois à un (1) an ou de l'une de ces deux peines seulement :

- les personnes qui utilisent des procédés, substances ou engins de pêche prohibés, ou dont les normes techniques ne sont pas conformes à celles autorisées par la législation en vigueur ; 
- les personnes qui se livrent à la pêche d'espèces dont la capture est prohibée ;

- les personnes qui se livrent à la pêche dans les zones ou périodes interdites ;

- les personnes qui se livrent à l'importation des poissons vivants sans autorisation.

ARTICLE 269 : Sont passibles d'une amende de dix mille francs à cent mille francs (10.000 F à $100.000 \mathrm{~F}$ ) et d'un emprisonnement de quinze (15) jours à six (6) mois ou de l'une de ces deux peines seulement :

- les personnes qui se livrent à des activités de pêche sans permis ou licence ;

- les personnes qui procèdent à la destruction ou à l'endommagement des embarcations, filets ou engins de pêche appartenant à autrui ;

- les personnes qui se livrent aux activités d'aquaculture sans autorisation.

ARTICLE 270 : Sont passibles d'une amende de cinq mille francs à cinquante mille francs (5.000 à $50.000 \mathrm{~F})$ les personnes qui vendent, transportent ou achètent des ressources piscicoles dont la pêche est interdite.

ARTICLE 271 : Dans un but d'éducation et en tenant compte des circonstances de l'espèce, le tribunal peut remplacer les sanctions prévues à l'article précédent par un travail d'intérêt commun.

\section{LIVRE V - DES DISPOSITIONS TRANSITOIRES ET FINALES}

ARTICLE 272 : Sont abrogées toutes dispositions antérieures contraires notamment :

- l'ordonnance 68-59 du 31 décembre 1968 relative à la conservation de la faune et à l'exercice de la chasse en Haute-Volta ;

- l'ordonnance $\mathrm{n}^{\circ}$ 81-009/PRES/CMRPN/ET du 9 mai 1981 portant organisation et réglementation de la pêche en Haute-Volta.

ARTICLE 273 : La présente loi sera exécutée comme loi de l'Etat.

Ainsi fait et délibéré en séance publique à Ouagadougou, le 31 janvier 1997

Pour le Président, le Deuxième Vice-Président

Jean-Marie SOMDA

Le Secrétaire de Séance

Batio Isaïe TRAORE

80 Textes et textes de loi sur la gestion des ressources naturelles au Burkina Faso 


\section{Annexe V}

\section{JO N01-2003 DU 02 JANVIER 2003 \\ LOI N 002-2001/AN PORTANT LOI D’ORIENTATION RELATIVE A LA GESTION DE L'EAU}

\section{L'ASSEMBLEE NATIONALE}

$\mathrm{Vu}$ la Constitution ;

$\mathrm{Vu}$ la Résolution $\mathrm{N}^{\circ}$ 01/97/AN du 07 juin 1997, portant validation du mandat des Députés ;

A délibéré en sa séance du 08 février 2001

et adopté la loi dont la teneur suit :

\section{CHAPITRE I - DE L'OBJET ET DU CHAMP D'APPLICATION SECTION I : DE L'OBJET}

ARTICLE 1 : L'eau est une ressource précieuse. Sa gestion durable constitue un impératif national.

La gestion de l'eau a pour but, dans le respect de l'environnement et des priorités définies par la loi :

- d'assurer l'alimentation en eau potable de la population ;

- de satisfaire ou de concilier les exigences de l'agriculture, de l'élevage, de la pêche et de l'aquaculture, de l'extraction des substances minérales, de l'industrie, de la production d'énergie, des transports, du tourisme, des loisirs ainsi que de toutes autres activités humaines légalement exercées ;

- de préserver et de restaurer la qualité des eaux ;

- de protéger les écosystèmes aquatiques ;

- de faire face aux nécessités de la santé, de la salubrité publique, de la sécurité civile et aux problèmes posés par les inondations et les sécheresses.

ARTICLE 2 : La loi reconnaît le droit de chacun à disposer de l'eau correspondant à ses besoins et aux exigences élémentaires de sa vie et de sa dignité.

Le Ministre chargé de l'eau, le Ministre chargé des affaires sociales et le Ministre chargé de la santé proposent et mettent en œuvre, dans le respect de leurs attributions respectives, en liaison avec les autres autorités publiques compétentes et les personnes privées intervenant dans le domaine de l'eau, les mesures nécessaires à l'exercice de ce droit.

ARTICLE 3 : La gestion de l'eau prend en considération, dans leur globalité et leurs relations réciproques, les données scientifiques et les solidarités de toute nature qui caractérisent les bassins hydrographiques.

ARTICLE 4: La diversité biologique des écosystèmes aquatiques, leur rôle dans la régulation et le renouvellement des ressources en eau, l'importance des fonctions sociales, économiques et culturelles auxquelles ils participent, confèrent à leur conservation un caractère prioritaire et d'intérêt général. 
Dans l'exercice de leurs compétences respectives, les personnes de droit public sont tenues, comme les personnes privées, de prendre en considération les exigences de la gestion durable des écosystèmes aquatiques.

\section{SECTION II : DU CHAMP D'APPLICATION}

ARTICLE 5 : L'eau est un élément du patrimoine commun de la Nation. Elle fait partie du domaine public.

ARTICLE 6 : Sous réserve des dispositions de l'article 8 ci-dessous, le domaine public de l'eau comprend l'eau dans ses divers états physiques et situations géomorphologiques ainsi que les ouvrages publics affectés ou nécessaires à sa gestion. Y sont inclus à ce titre :

$1^{\circ}$ les cours d'eau ;

$2^{\circ}$ les lacs naturels ou artificiels, les étangs, les mares et d'une manière générale, les étendues d'eau ;

$3^{\circ}$ les espaces où la présence de l'eau, sans être permanente, est régulière et empêche ou conditionne directement l'exploitation à des fins agricoles ;

$4^{\circ}$ les eaux souterraines ;

$5^{\circ}$ l'eau atmosphérique ;

$6^{\circ}$ les sources, puits, forages, abreuvoirs et autres points d'eau affectés à l'usage du public ou à un service public ainsi que leurs périmètres de protection immédiate, délimités en application de l'article 34, alinéa 1 ;

$7^{\circ}$ les digues, les barrages, les chaussées, les écluses et leurs dépendances ou ouvrages annexes ;

$8^{\circ}$ les canaux d'irrigation, d'assainissement et de drainage ;

$9^{\circ}$ les aqueducs, les canalisations, les dérivations et les conduites d'eau ; les réservoirs, les stations de traitement d'eau potable, les stations d'épuration des eaux usées et, d'une manière générale, les ouvrages hydrauliques affectés à l'usage du public ou à un service public ainsi que les installations et les terrains qui en dépendent ;

ARTICLE 7 : Nonobstant les dispositions de l'article 6 ci-dessus, l'eau recueillie dans un ouvrage privé et destinée à un usage domestique ne fait pas partie du domaine public.

Il en est de même des piscines, des étangs, des citernes et des bassins d'agrément construits ou aménagés par les personnes privées sur un fonds privé.

ARTICLE 8 : Dans le cas des cours d'eau, le domaine public inclut le lit, identifié par la présence de l'eau ou de traces apparentes résultant de l'écoulement des eaux; les berges, jusqu'à la limite atteinte par les eaux avant débordement, et les francs-bords.

Les francs-bords sont constitués par les terrains compris dans une bande délimitée de part et d'autre des berges.

ARTICLE 9: Les cours d'eau, les étendues d'eau, mentionnés à l'article 6, alinéas 1,2 et 3 sont inscrits dans une nomenclature établie par décret pris en Conseil des Ministres après une enquête publique conduite sous l'autorité du Ministre chargé de l'eau.

82 Textes et textes de loi sur la gestion des ressources naturelles au Burkina Faso 
ARTICLE 10 : Des arrêtés conjoints des Ministres chargés respectivement de l'eau, des domaines et de la santé, pris après enquête publique conduite sous l'autorité du Ministre chargé de l'eau et conformément à une procédure fixée par décret pris en Conseil des Ministres, déterminent les limites des dépendances du domaine public de l'eau et, en particulier :

- des cours d'eau et de leurs francs-bords ;

- des étendues d'eau mentionnées à l'article 6, alinéas 2 et 3 ;

- des périmètres de protection immédiate mentionnés à l'article 6 , alinéa 6 ;

- des ouvrages et des terrains mentionnés à l'article 6 , alinéas 7,8 et 9 .

ARTICLE 11 : Des décrets pris en Conseil des ministres déterminent les modalités de l'indemnisation des propriétaires et autres titulaires de droits fonciers ayant subi un préjudice direct, matériel et certain du fait du classement de leurs terrains parmi les dépendances du domaine public de l'eau à la suite d'une modification des limites de ce dernier, que cette modification résulte des dispositions de la présente loi ou d'un changement artificiel ou naturel du cours ou du régime des eaux.

Les décrets mentionnés à l'alinéa précédent fixent également les conditions dans lesquelles peuvent être indemnisées les personnes auxquelles l'application effective des dispositions législatives relatives au domaine public de l'eau occasionnerait un préjudice direct, matériel et certain en raison de la remise en cause de droits réputés acquis par référence à des règles coutumières ou à des usages antérieurs.

\section{CHAPITRE II - DE L' ADMINISTRATION DE L'EAU SECTION I : DES STRUCTURES}

ARTICLE 12 : Il est créé, auprès du Ministère chargé de l'Eau, un Conseil national de l'eau. Il a un caractère consultatif.

Un décret pris en Conseil des Ministres fixe ses attributions et règle les modalités de sa composition, de son organisation et de son fonctionnement.

ARTICLE 13 : Le Ministère chargé de l'eau est le garant institutionnel de la gestion intégrée des ressources en eau.

ARTICLE 14 : Les décisions relatives à la gestion de l'eau sont prises par les autorités locales, dans le cadre de la collectivité ou de la circonscription administrative dont le champ territorial de compétences est le plus restreint, sous réserve qu'aucune considération d'intérêt général ou liée à la nécessité de satisfaire dans les meilleures conditions, les besoins en eau de toute nature ne s'y oppose

ARTICLE 15 : Le Ministre chargé de l'eau veille à ce que les populations concernées par un aménagement hydraulique ou une mesure de gestion de l'eau reçoivent une information appropriée.

Il organise et définit les modalités d'une concertation permettant d'améliorer la gestion de l'eau dans le cadre des collectivités territoriales et des communautés villageoises.

ARTICLE 16 : Le Ministre chargé de l'eau propose au Gouvernement, après avis du Conseil national de l'eau, toutes mesures tendant à favoriser la mise en œuvre des dispositions énoncées aux articles 14 et 15 ci-dessus. 


\section{SECTION II : DES INSTRUMENTS ET DU CADRE DE LA GESTION DE L'EAU}

\section{PARAGRAPHE 1 : DU PLAN D'ACTION DE L'EAU}

ARTICLE 17 : Un Plan d'action de l'eau est élaboré sous l'autorité du Ministre chargé de l'eau et approuvé par décret pris en Conseil des Ministres.

\section{PARAGRAPHE 2 : DES BASSINS HYDROGRAPHIQUES}

ARTICLE 18 : Le bassin hydrographique est le cadre approprié de planification et de gestion de la ressource en eau.

La coordination des actions publiques et la concertation s'y inscrivent afin de préparer et de mettre en œuvre, dans les conditions optimales de rationalité, les orientations et les décisions prises dans le domaine de l'eau.

ARTICLE 19 : Le territoire national comprend quatre bassins nationaux :

- le bassin de la Comoé ;

- le bassin du Mouhoun ;

- le bassin du Nakanbé ;

- le bassin du Niger.

Les limites des bassins et des sous-bassins qui peuvent leur être rattachés sont fixées par décret pris en Conseil des Ministres.

ARTICLE 20 : Sur proposition du Ministre chargé de l'eau, le Gouvernement détermine par décret les structures dont l'institution pourrait être envisagée dans les bassins, ainsi que les missions et attributions qui leur seront confiées pour la gestion de l'eau.

L'espace de compétence de ces structures prend en compte les critères scientifiques, techniques, administratifs ou socio-économiques.

PARAGRAPHE 3 : DES SCHEMAS D'AMENAGEMENT ET DE GESTION DE L'EAU

ARTICLE 21 : Les schémas d'aménagement et de gestion de l'eau relèvent de la compétence de l'Etat.

Les schémas d'aménagement et de gestion de l'eau fixent dans le cadre, selon le cas, d'un bassin, d'un groupement de bassins, d'un ou plusieurs sous-bassins, d'une portion de cours d'eau ou d'un système aquifère, les orientations d'une gestion durable de l'eau.

Les programmes et les décisions administratives dans le domaine de l'eau doivent être compatibles ou rendus compatibles avec les dispositions du schéma.

ARTICLE 22: Les modalités d'application des articles 17 et 21 ci-dessus et en particulier les dispositions relatives au contenu, à l'élaboration, à l'approbation, à la mise en ouvre et au suivi du Plan d'action de l'eau et des schémas d'aménagement et de gestion de l'eau sont déterminées par décrets pris en Conseil des Ministres.

84 Textes et textes de loi sur la gestion des ressources naturelles au Burkina Faso 


\section{CHAPITRE III - DU REGIME DE L'EAU SECTION I : DU POUVOIR DE CONTROLE ET DE REPARTITION}

ARTICLE 23 : Lorsqu'une sécheresse grave ou d'autres circonstances exceptionnelles ne permettent pas de satisfaire l'intégralité des besoins en eau, le Gouvernement détient, sans préjudice des mesures qui peuvent être prises en application de l'article 29 ci-dessous, un droit de contrôle et de répartition, mis en œuvre selon les modalités définies par décret pris en Conseil des Ministres. Il dispose, ainsi que, par délégation, le Ministre chargé de l'eau, des mêmes prérogatives dans une localité ou une partie du territoire où il s'avère impossible ou très difficile d'assurer dans des conditions normales l'exercice des diverses activités consommatrices d'eau.

Dans tous les cas où sont prises des mesures de contrôle et de répartition, les besoins en eau qui correspondent à l'alimentation des populations et aux conditions élémentaires de la vie et de la dignité sont considérés comme prioritaires.

\section{SECTION II : DE LA REGLEMENTATION DES UTILISATIONS DE L'EAU}

ARTICLE 24: Sont soumis à autorisation ou à déclaration les aménagements hydrauliques et, d'une manière générale, les installations, ouvrages, travaux et activités réalisés par toute personne physique ou morale, publique ou privée, et entraînant, selon le cas :

- des prélèvements d'eau superficielle ou souterraine, restitués ou non ;

- une modification du niveau ou du mode d'écoulement des eaux ;

- des déversements, écoulements, rejets ou dépôts directs ou indirects, chroniques ou épisodiques, même non polluants.

ARTICLE 25 : Les installations, ouvrages, travaux et activités visés à l'article précédent sont soumis à autorisation ou à déclaration suivant leur nature, leur localisation, leur importance ou la gravité de leurs effets sur la ressource en eau et les écosystèmes aquatiques.

ARTICLE 26: Sont soumis à autorisation les installations, ouvrages, travaux et activités susceptibles de présenter des dangers pour la santé et la sécurité publique, de réduire la ressource en eau, de modifier substantiellement le niveau, le mode d'écoulement ou le régime des eaux, de porter gravement atteinte à la qualité ou à la diversité des écosystèmes aquatiques.

L'autorisation fixe, en tant que de besoin, les prescriptions imposées au bénéficiaire en vue de supprimer, réduire ou compenser les dangers ou les incidences sur l'eau et les écosystèmes aquatiques.

ARTICLE 27 : Les installations, ouvrages, travaux et activités mentionnés à l'article 24 de la présente loi qui ne présentent pas des dangers ou des incidences sur l'eau ou les écosystèmes aquatiques et impliquant un régime d'autorisation, sont soumis à déclaration.

Les installations, ouvrages, travaux et activités soumis à déclaration doivent respecter les règles générales édictées en vue de préserver la santé, la salubrité, la sécurité, la qualité des eaux et pour assurer la conservation des écosystèmes aquatiques. 
ARTICLE 28: Lorsqu'une installation, un ouvrage, un travail ou une activité soumise à autorisation ou à déclaration fonctionne ou s'exerce, sans autorisation ou sans avoir été déclaré, le maître d'ouvrage ou l'exploitant encourt, indépendamment des éventuelles poursuites pénales ou des indemnités dues au titre de sa responsabilité civile, une mesure de suspension prononcée par le Ministre chargé de l'eau ou son représentant.

ARTICLE 29 :Sans préjudice des mesures prises en cas de circonstances exceptionnelles prévues par l'article 23 ci-dessus, le Ministre chargé del'eau peut réglementer ou interdire certaines utilisations de l'eau afin de prévenir un risque de pénurie, ou de prévenir ou faire cesser les conséquences d'un accident ou d'une période de sécheresse.

ARTICLE 30 : Des décrets pris en Conseil des Ministres déterminent les modalités d'application des dispositions et la nomenclature des installations, ouvrages, travaux et activités soumis, selon le cas, à autorisation ou à déclaration, les procédures qui leur correspondent, les règles générales mentionnées à l'article 24 , alinéa 2 , ainsi que les conditions dans lesquelles le Ministre chargé de l'eau exerce les pouvoirs que lui conferent les articles 28 et 29 ci-dessus.

\section{SECTION III : DE LA PROTECTION DE L'EAU}

\section{PARAGRAPHE 1 : DES SERVITUDES}

ARTICLE 31 : Les servitudes de droit privé et de droit public qui affectent ou ont une incidence sur l'eau, son mode d'écoulement et son régime, demeurent soumises aux dispositions légales et réglementaires qui leur sont applicables.

ARTICLE 32 : Sur proposition du Ministre chargé de l'eau, un décret pris en Conseil des Ministres institue une servitude de rétention.

Ce décret détermine dans quelles conditions les propriétaires, locataires ou exploitants d'un terrain non bâti pourront être tenus de conserver temporairement ou de limiter l'écoulement des eaux se trouvant ou circulant sur leurs fonds. Il fixera également les modalités de l'indemnisation éventuelle des propriétaires ou des ayants droit dans le cas où ceux-ci subiraient, du fait de l'institution de la servitude de rétention, un préjudice direct, matériel et certain.

\section{PARAGRAPHE 2: DE LA PROTECTION DES PRELEVEMENTS D'EAU DESTINEE A LA CONSOMMATION HUMAINE}

ARTICLE 33 : En vue d'assurer la protection de la qualité des eaux, l'autorisation des travaux, des installations, des ouvrages réalisés pour le prélèvement d'eau superficielle ou souterraine, destinée à la consommation humaine, ou l'autorisation de ces prélèvements eux-mêmes, délimite autour du point de prélèvement, un périmètre de protection immédiate, un périmètre de protection rapprochée et, en tant que de besoin, un périmètre de protection éloignée.

Ces périmètres sont également déterminés dans le cas des prélèvements soumis à déclaration, dès lors que l'eau prélevée est totalement ou partiellement destinée à la consommation humaine.

ARTICLE 34 : Les terrains inclus dans le périmètre de protection immédiate sont acquis en pleine propriété par l'Etat ou le concessionnaire du service public de distribution, 
qui ont en charge de les clôturer et de veiller à ce qu'ils soient exclusivement affectés au prélèvement de l'eau et régulièrement entretenus à cette fin.

A l'intérieur des périmètres de protection rapprochée, les dépôts, installations et activités de nature à nuire directement ou indirectement à la qualité de l'eau ou à la rendre impropre à la consommation humaine, sont interdits.

L'interdiction porte, en particulier, sur les dépôts d'ordures, d'immondices et de détritus; l'épandage du fumier; les dépôts d'hydrocarbures et de toutes substances présentant des risques de toxicité, notamment de produits chimiques, de pesticides et d'engrais ; le forage de puits ; l'extraction de substances minérales.

En complément des périmètres de protection immédiate et rapprochée, l'autorité compétente peut délimiter un périmètre de protection éloignée à l'intérieur duquel les dépôts, installations et activités mentionnés à l'alinéa précédent peuvent être réglementés afin de prévenir les dangers de pollution qu'ils présentent pour les eaux prélevées.

ARTICLE 35 : L'autorité compétente peut délimiter des aires de protection autour des retenues de barrages, des lacs, des mares et, d'une manière générale, des étendues d'eau destinées, au moins partiellement, à la consommation humaine. Ces aires peuvent également être instituées pour protéger des zones d'alimentation des nappes souterraines.

Outre les interdictions et règles édictées à l'article 34 ci-dessus, l'acte de délimitation peut également réglementer des activités telles que l'abreuvement, le parcage et la circulation des animaux, l'édification de constructions ou de bâtiments à usage d'habitation ou non.

ARTICLE 36 : Des décrets pris en Conseil des Ministres déterminent les modalités d'application des articles 33 à 35 et en particulier les procédures de délimitation des aires et des périmètres de protection rapprochée et éloignée, y compris lorsqu'ils concernent des points de prélèvement existant à la date de promulgation de la présente loi, ainsi que les conditions dans lesquelles les propriétaires ou occupants de terrains concernés peuvent être indemnisés dans le cas où ils subissent, de ce fait, un préjudice direct, matériel et certain.

PARAGRAPHE 3 : DES DISPOSITIONS APPLICABLES AUX ACTIVITES AYANT UNE INCIDENCE SUR LA RESSOURCE EN EAU

\section{ARTICLE 37 : Sont interdites les pratiques et techniques agricoles susceptibles d'avoir une incidence négative sur le cycle hydrologique ou la qualité de l'eau.}

Une réglementation des activités pastorales comportant le cas échéant des restrictions à la circulation des animaux, peut être édictée selon la même procédure.

ARTICLE 38 : Un décret pris en Conseil des Ministres détermine les zones à l'intérieur desquelles l'édification de constructions ou de bâtiments, à usage d'habitation ou non, est interdite ou subordonnée à l'observation de prescriptions spéciales en raison des risques d'atteinte à la qualité de l'eau, des dangers pour la population, des difficultés prévisibles d'approvisionnement en eau ou encore des obstacles à la réalisation de l'assainissement. 
ARTICLE 39 : La réalisation des installations, ouvrages, travaux et activités soumis à autorisation en application de l'article 26 ci-dessus, peut donner lieu à l'élaboration d'une étude d'impact permettant de déterminer leurs incidences sur l'eau et les écosystèmes aquatiques.

Dans le cas où l'étude d'impact est obligatoire, son absence ou son insuffisance manifeste entraîne le refus de l'autorisation.

Un décret pris en Conseil des Ministres précise les modalités d'application du présent article.

\section{SECTION IV : DE LA PROTECTION DES ECOSYSTEMES AQUATIQUES}

ARTICLE 40 : Les ouvrages construits dans le lit des cours d'eau doivent maintenir un débit minimal garantissant la vie aquatique et les priorités définies à l'article 23 ci-dessus. Lorsqu'ils sont implantés dans des cours d'eau fréquentés par des poissons migrateurs, ils doivent en outre être équipés de dispositifs de franchissement.

ARTICLE 41 : Dans les parcs nationaux, les réserves de faune totales ou partielles, les réserves de la biosphère et les sanctuaires qui englobent tout ou partie d'un ou plusieurs écosystèmes aquatiques, ainsi que dans les zones humides d'importance internationale inscrites sur la liste prévue par la Convention de Ramsar du 02 février 1971, les actions susceptibles de porter atteinte à l'équilibre de ces écosystèmes ou d'affecter leur diversité biologique, sont réglementées et le cas échéant interdites. Sont visés notamment les utilisations des eaux entraînant une modification de leur niveau, de leur mode d'écoulement ou de leur régime, l'épandage à quelque fin que ce soit de produits chimiques et en particulier de pesticides agricoles, les rejets d'effluents ou de substances toxiques, le déversement ou l'écoulement d'eaux usées et le dépôt d'immondices ou de déchets domestiques ou industriels.

La réglementation ou l'interdiction peut, en tant que de besoin, porter sur des actions réalisées ou envisagées à l'extérieur de l'aire protégée ou de la zone humide.

ARTICLE 42 : Les zones humides d'importance internationale inscrites sur la liste prévue par la Convention de Ramsar du 02 février 1971 doivent être dotées d'un plan de gestion.

Le plan de gestion est réalisé sous l'autorité des Ministères chargés de l'eau et de l'environnement.

\section{CHAPITRE IV - DU REGIME DES SERVICES PUBLICS DANS LE DOMAINE DE LEAU ET DU CONTROLE DE SES UTILISATIONS A DES FINS ECONOMIQUES}

ARTICLE 43 : Dans tout ou partie du territoire d'une collectivité territoriale, l'Etat peut déléguer à celle-ci, aux conditions qu'il définit conformément à la loi, certaines de ses compétences relatives à l'utilisation de l'eau.

Cette délégation concerne la gestion du service public de distribution d'eau potable, ou des utilisations de l'eau à des fins agricoles, aquacoles, pastorales, industrielles, touristiques ou de production d'énergie.

Elle peut porter également sur l'assainissement, entendu comme le traitement et l'évacuation des eaux usées et, le cas échéant, l'évacuation des eaux pluviales. 
ARTICLE 44 : La personne publique ou privée qui, dans le cadre d'un contrat passé avec l'Etat ou une collectivité territoriale déléguée, assure la distribution de l'eau, peut également prendre en charge le service de l'assainissement. Ce dernier est alors géré selon les mêmes modalités que la distribution de l'eau ou en application de dispositions qui lui sont propres.

ARTICLE 45 : L'Etat ou la collectivité territoriale déléguée gère le service public de distribution d'eau, lui-même ou sous sa responsabilité, en régie ou dans le cadre d'un contrat de gestion ou de gérance, soit par voie de concession ou d'affermage.

Un décret pris en Conseil des Ministres précise les modes de gestion et leurs conditions d'application.

ARTICLE 46 : Quel que soit le mode de gestion retenu en matière de distribution d'eau et d'assainissement, les personnes publiques ou privées qui en ont la charge sont soumises aux règles générales applicables aux services publics. Elles se conforment, en particulier, au principe d'égalité entre les usagers, au principe de continuité, selon lequel le service doit fonctionner de manière régulière et ininterrompue et au principe d'adaptation du service à l'évolution des besoins collectifs et aux exigences de l'intérêt général.

Elles sont responsables de la qualité de l'eau distribuée dont les normes de potabilité seront fixées par arrêté conjoint des Ministres chargés de l'eau et de la santé.

\section{CHAPITRE V - DU FINANCEMENT DU SECTEUR DE L'EAU SECTION I : DU SYSTEME DE FINANCEMENT}

ARTICLE 47 : L'utilisation de l'eau exige de chacun qu'il participe à l'effort de la Nation pour en assurer la gestion.

Ceux qui, par leur activité, rendent nécessaires ou utiles des interventions publiques ou privées en vue de préserver ou de restaurer la qualité de l'eau, de répondre aux besoins correspondant aux utilisations qui en sont faites ou d'assurer la conservation des écosystèmes aquatiques, supportent la charge de ces interventions ou contribuent à leur financement.

ARTICLE 48 : Les personnes physiques ou morales dont l'activité est de nature à provoquer ou aggraver la pollution de l'eau ou la dégradation du milieu aquatique contribuent au financement des mesures que l'Etat et les collectivités publiques doivent prendre pour lutter contre cette pollution, en compenser les effets et pour assurer la conservation des écosystèmes aquatiques.

Les contributions ainsi définies sont proportionnées à l'importance de la pollution ou de la dégradation en cause. Elles peuvent être réduites à raison des dispositions prises par les redevables pour y remédier et n'excluent pas le bénéfice d'une aide publique tendant aux mêmes fins.

Le versement d'une contribution déterminée en application des dispositions ci-dessus ne fait pas obstacle à l'engagement de la responsabilité civile ou pénale du redevable dans le cas où son activité serait à l'origine d'un dommage ou constituerait une infraction.

ARTICLE 49 : Les personnes physiques ou morales qui utilisent de l'eau à des fins autres que domestiques peuvent être assujetties au versement d'une contribution financière 
assise sur le volume d'eau prélevé, consommé ou mobilisé ; cette contribution doit en priorité servir au financement du secteur de l'eau.

La détermination des personnes assujetties ou exemptées, des activités concernées, du montant et des règles administratives et comptables applicables à cette contribution prend en considération l'importance sociale, économique, culturelle et écologique de l'activité en cause, les revenus et profits de toute nature pouvant en résulter, ainsi que les charges collectives qu'elle impose, notamment à l'Etat et aux autres personnes publiques, en matière de gestion de l'eau.

Les modalités d'application des dispositions qui précèdent font l'objet d'un décret pris en Conseil des Ministres pris sur proposition conjointe des Ministres chargés respectivement des finances, de l'eau et du ou des Ministres dont les attributions correspondent à l'activité pour laquelle une contribution financière est envisagée.

ARTICLE 50 : Sur proposition des Ministres chargés des finances et de l'eau, un décret pris en Conseil des Ministres précise les utilisations domestiques, en fixe les seuils et définit le cas échéant les conditions dans lesquelles certaines utilisations domestiques peuvent être soumises à une contribution financière.

\section{SECTION II : DU REMBOURSEMENT DES DEPENSES EXPOSEES PAR DES PERSONNES PUBLIQUES EN CAS DE POLLUTION ACCIDENTELLE}

ARTICLE 51 : En cas de pollution accidentelle de l'eau, les personnes publiques intervenues matériellement ou financièrement ont droit au remboursement par la ou les personnes à qui incombe la responsabilité de l'accident, des frais d'enquête et d'expertise exposés par elles ainsi que des dépenses effectuées pour atténuer ou éviter l'aggravation des dommages. Le remboursement des sommes dues s'effectue sans préjudice de l'indemnisation des autres dommages.

\section{CHAPITRE VI - DES DISPOSITIONS PENALES}

ARTICLE 52 : Les infractions aux prescriptions de la présente loi constituent des contraventions ou des délits et sont punies des sanctions prévues par les articles 54 à 67 ci-après, sauf lorsqu'il en est disposé autrement.

Ces peines sont portées au double en cas de récidive, sauf dispositions contraires.

ARTICLE 53 : Les infractions aux dispositions de la présente loi sont constatées par les officiers de police judiciaire, les agents de la police municipale et les agents assermentés appartenant aux services de l'Etat chargés de l'eau, de la santé et de l'environnement.

Les officiers de police judiciaire et les agents assermentés procèdent aux enquêtes, constatent les infractions, opèrent les saisies conformément au code de procédure pénale. Ils dressent un procès-verbal des faits constatés.

Les procès-verbaux font foi jusqu'à preuve du contraire.

ARTICLE 54 : Quiconque a jeté, déversé ou laissé s'écouler dans les eaux superficielles ou souterraines, directement ou indirectement, une ou des substances quelconques dont l'action ou les réactions ont entraîné ou sont susceptibles d'entraîner, même provisoirement, des effets nuisibles sur la santé ou des atteintes à la diversité biologique ou à l'équilibre des écosystèmes aquatiques, sera puni d'une amende de 50000 FCFA 
à 5000000 FCFA et d'un emprisonnement de onze jours à deux mois, ou de l'une de ces deux peines seulement. Lorsque l'opération de rejet a été autorisée, ou occasionnée par une activité autorisée par l'administration, les dispositions de cet alinéa ne s'appliquent que si les prescriptions de l'autorisation n'ont pas été respectées.

ARTICLE 55 : Est puni d'un emprisonnement de quinze jours à un mois et d'une amende de 50000 FCFA à 500000 FCFA ou de l'une de ces deux peines seulement, quiconque effectue des prélèvements d'eau en violation de l'obligation de requérir l'autorisation exigée en application des articles 24 et 26.

ARTICLE 56 : Est puni d'une amende de 5000 FCFA à 50000 FCFA quiconque effectue des prélèvements d'eau sans avoir souscrit à la déclaration prescrite par l'article 27.

ARTICLE 57 : Est puni d'un emprisonnement de un mois à trois mois et d'une amende de 100000 FCFA à 5000000 FCFA ou de l'une de ces deux peines seulement, quiconque a, soit construit, modifié ou exploité un ouvrage ou une installation, soit réalisé des travaux ou exercé une activité sans l'autorisation requise en application des articles 24 et 26.

En cas de condamnation, le tribunal peut décider la cessation des travaux ou des activités, l'interdiction d'utiliser l'installation ou l'ouvrage, ou la suspension de son fonctionnement.

Il peut également ordonner la destruction de l'installation ou de l'ouvrage assorti le cas échéant de la remise des lieux en l'état.

ARTICLE 58: Est puni d'une amende de 50000 FCFA à 250000 FCFA quiconque a, soit construit un ouvrage ou une installation, soit réalisé des travaux ou exercé une activité sans respecter les prescriptions imposées par l'acte d'autorisation.

En cas de condamnation, le tribunal peut ordonner toute mesure prévue à l'article 57, alinéas 2 et 3 .

ARTICLE 59 : Est puni d'un emprisonnement de quinze jours à un mois et d'une amende de 50000 FCFA à 250000 FCFA ou de l'une de ces deux peines seulement, quiconque a, soit construit, modifié ou exploité un ouvrage ou une installation, soit réalisé des travaux ou exercé une activité sans avoir souscrit à la déclaration prescrite par les articles 24 et 27.

Est puni d'une amende de 50000 FCFA quiconque a, soit construit ou exploité un ouvrage ou une installation, soit réalisé des travaux ou exercé une activité soumise à déclaration en violation des règles générales prévues à l'article 27, alinéa 2.

ARTICLE 60 : Est puni d'un emprisonnement de un à trois mois et d'une amende de 250000 FCFA à 8000000 FCFA ou de l'une de ces deux peines seulement, quiconque exploite un ouvrage ou une installation, réalise des travaux ou exerce une activité en violation d'une mesure de suspension décidée en application de l'article 28.

Les mêmes peines sont encourues par quiconque exploite un ouvrage ou une installation, réalise des travaux ou exerce une activité en violation d'une mesure de cessation, d'interdiction, de suspension ou de destruction ordonnée par un tribunal en application de l'article 57, alinéas 2 et 3 ou de l'article 58, alinéa 2. 
ARTICLE 61 : Est puni d'une amende de 50000 FCFA quiconque aura utilisé de l'eau en violation d'une interdiction ou d'une mesure de restriction édictée en application de l'article 29.

ARTICLE 62: Est puni d'une amende de 50000 FCFA quiconque a, dans un périmètre de protection rapproché d'un point de prélèvement des eaux, réalisé des dépôts, construit ou exploité une installation, ou exercé une activité en violation d'une interdiction édictée en application de l'article 34 ci-dessus.

En cas de récidive, la peine encourue est une amende de 50000 FCFA à 100000 FCFA.

Les sanctions prévues au présent article s'appliquent également aux personnes qui, dans un périmètre de protection éloigné d'un point de prélèvement des eaux ou dans une aire de protection d'un plan d'eau affecté à la consommation humaine, auront contrevenu à une règle ou à une mesure d'interdiction édictée en application de l'article 34, alinéa 3 ou de l'article 35 .

ARTICLE 63 : Est puni d'une amende de 50000 FCFA à 250000 FCFA quiconque exerce une activité agricole ou pastorale en violation d'une mesure d'interdiction ou d'une règle édictée en application de l'article 37.

ARTICLE 64 : Quiconque édifie une construction ou un bâtiment en violation d'une interdiction ou d'une règle édictée en application de l'article 38 encourt les sanctions prévues par la législation en vigueur en matière d'urbanisme.

ARTICLE 65: Est puni d'une amende de 50000 FCFA à 250000 FCFA quiconque construit ou exploite un ouvrage en violation des obligations imposées par l'article 40.

ARTICLE 66 : Est puni d'un emprisonnement de un à trois mois et d'une amende de 100000 FCFA à

5000000 FCFA ou de l'une de ces deux peines seulement, quiconque réalise, en violation d'une mesure d'interdiction ou d'une règle édictée en application de l'article 41, une action susceptible de porter atteinte à l'équilibre d'un écosystème ou d'affecter sa diversité biologique.

Le tribunal pourra également imposer au condamné de procéder à la restauration du milieu aquatique, le cas échéant sous astreinte.

ARTICLE 67 : Est puni des peines prévues à l'article 55 de la présente loi, quiconque viole l'obligation de souscrire la déclaration qui lui est imposée en application de l'article 68, alinéa 1.

Les mêmes peines sont encourues par quiconque ne respecte pas les prescriptions d'un arrêté ministériel pris en application de l'article 69.

\section{CHAPITRE VII - DES DISPOSITIONS TRANSITOIRES}

ARTICLE 68 : Un décret pris en Conseil des Ministres sur rapport du Ministre chargé de l'eau détermine, en tant que de besoin :

92 Textes et textes de loi sur la gestion des ressources naturelles au Burkina Faso 
$1^{\circ}$ Les conditions, notamment de délais, dans lesquelles les propriétaires ou exploitants d'installations ou d'ouvrages visés aux articles 24 à 28 et 30 sont tenus de déclarer au Ministre chargé de l'eau les installations et ouvrages autorisés, construits ou exploités avant la promulgation de la loi ;

$2^{\circ}$ Les dispositions de tous ordres applicables à ces installations et ouvrages, et en particulier le délai au terme duquel ils devront satisfaire aux obligations légales ; ainsi que, dans les cas où leur mise en conformité s'avérerait impossible pour des raisons de droit ou de fait, les conséquences juridiques, administratives et financières d'une telle situation ;

$3^{\circ}$ Les mesures administratives qui peuvent être prises à l'encontre des propriétaires ou exploitants qui n'ont pas souscrit à la déclaration exigée à l'alinéa 1 ci-dessus, sans préjudice des sanctions pénales prévues à l'article 67 ci-dessus.

Les dispositions du présent article ne sont pas applicables aux extensions ou aux modifications d'installations ou d'ouvrages postérieurs à la promulgation de la présente loi.

ARTICLE 69: Avant l'entrée en vigueur des obligations résultant des articles 24 et 26 et pendant une durée de trois ans au plus à compter de la promulgation de celleci, le Ministre chargé de l'eau peut, par arrêté motivé, soumettre certains ouvrages, installations, travaux et activités à des prescriptions qu’il détermine. Les propriétaires, exploitants ou personnes concernées doivent satisfaire aux obligations qui leur sont ainsi imposées dans un délai de trois mois à compter de l'arrêté ministériel.

Ces prescriptions ne peuvent être édictées qu'en vue de réaliser une gestion durable de l'eau dans le strict respect des libertés et des droits garantis par la Constitution.

ARTICLE 70 : La présente loi qui abroge toutes dispositions antérieures contraires sera exécutée comme loi de l'Etat.

Ainsi fait et délibéré en séance publique à Ouagadougou, le 08 février 2001

\section{Le Président}

\section{Mélégué TRAORE}

Le Secrétaire de séance

\section{Françoise Odette COULIBALY}




\section{Annexe VI}

\section{JO N01 DU 10 JANVIER 2003 \\ LOI Nº34-2002/AN LOI D'ORIENTATION RELATIVE AU PASTORALISME AU BURKINA FASO}

\section{L'ASSEMBLEE NATIONALE}

VU la Constitution ;

VU la résolution $n^{\circ} 001-2002 / A N$ du 05 juin 2002, portant validation du mandat des députés ;

a délibéré en sa séance du 14 novembre 2002

et adopté la loi dont la teneur suit :

\section{TITRE I : DISPOSITIONS GENERALES}

\section{CHAPITRE 1 : OBJET ET CHAMP D'APPLICATION}

ARTICLE 1 : La présente loi fixe les principes et les modalités d'un développement durable, paisible et intégré des activités pastorales, agropastorales et sylvopastorales.

ARTICLE 2 : Sont concernées par les dispositions de la présente loi, les activités d'élevage des espèces bovine, ovine, caprine, caméline, asine et équine.

\section{CHAPITRE 2 : DEFINITION DES TERMES}

ARTICLE 3 : Au sens de la présente loi, on entend par :

- pastoralisme : toute activité d'élevage consistant à assurer l'alimentation et l'abreuvement des animaux par l'exploitation directe des ressources naturelles sur des espaces déterminés et impliquant la mobilité des animaux.

Le pastoralisme désigne également les activités associant de manière complémentaire l'élevage, l'agriculture et la sylviculture.

- pasteur : toute personne qui exerce à titre d'occupation principale l'activité d'élevage pastoral et qui en tire l'essentiel de ses revenus, qu'il soit propriétaire de tout ou partie du troupeau.

- ressources pastorales : ressources végétales, hydriques et minérales exploitées dans le cadre de l'élevage pastoral ; elles sont comprises soit dans les espaces affectés à la pâture des animaux, soit dans les espaces ouverts à la pâture des animaux.

- espaces pastoraux : espaces affectés et espaces ouverts à la pâture des animaux.

Constituent des espaces affectés à la pâture des animaux, les espaces dont la destination principale est l'exercice d'activités pastorales. Ce sont :

- les espaces pastoraux d'aménagement spécial ;

- les espaces de terroir réservés à la pâture ;

- les espaces de cultures fourragères destinés à la pâture directe des animaux.

- les espaces pastoraux d'aménagement spécial ou zones pastorales : espaces identifiés comme tels par les schémas national, régional ou provincial d'aménagement du territoire ou par le schéma directeur d'aménagement et affectés à la réalisation d'opérations de mise en valeur pastorale. 
- les espaces de terroir réservés à la pâture : espaces ruraux traditionnellement affectés à la réalisation d'activités pastorales. Ils comprennent notamment les pâturages villageois ou inter-villageois, les espaces de cure salée et les espaces de bourgou.

Sont également considérés comme des espaces de terroirs réservés à la pâture, les pâturages ou espaces ruraux traditionnels faisant l'objet d'opérations locales de préservation ou de mise en valeur à des fins pastorales, dans le cadre des actions de gestion de l'espace et des ressources naturelles.

- les espaces de cure salée : espaces caractérisés par la teneur spécifique des sols en sels minéraux et utilisés périodiquement pour l'enrichissement de l'alimentation des animaux.

- espaces de bourgou dits bourgoutières : prairies semi-aquatiques dominées par la graminée fourragère Echinochloa stagnina ou bourgou.

- les espaces ouverts à la pâture : espaces dont la destination principale est autre que pastorale, mais supportant des droits d'usage pastoraux. Il s'agit notamment :

- des espaces forestiers ouverts à la pâture ;

- des terres agricoles laissées en jachère ;

- des champs de cultures après récoltes.

- des espaces forestiers ouverts à la pâture : espaces constitués d'une part, des espaces de forêts protégées, et d'autre part des espaces de forêts classées, uniquement lorsque les actes de classement ou les plans d'aménagement de ces dernières autorisent la pâture conformément aux dispositions de la législation forestière en vigueur.

Les espaces de forêts protégées non mis en culture sont considérés comme espaces sylvopastoraux. Ils sont utilisés sans autorisation préalable pour la pâture des animaux.

- jachères : espaces de cultures temporairement laissés au repos en vue de la restauration naturelle de la fertilité des sols.

- champs de cultures après récoltes : espaces agricoles envisagés comme espaces de pâturage uniquement après les périodes de récoltes en vue de l'exploitation des résidus de récoltes.

- pistes d'accès : voies affectées à la circulation des animaux et permettant l'accès des troupeaux aux points d'abreuvement, aux pâturages, aux infrastructures zoo-sanitaires et aux habitations.

- pistes de transhumance : voies affectées à la circulation des animaux en transhumance en vue de l'exploitation des points d'eau, des pâturages et des cures salées.

- pistes de commercialisation : voies affectées à la circulation des animaux et permettant de les convoyer à pied des zones de production vers les centres de consommation et/ou les marchés à bétail.

- vaine pâture : droit pour un éleveur de faire paître son bétail sur les espaces naturels et les espaces non clos d'autrui après la récolte, sous réserve du consentement de l'exploitant.

- transhumance : déplacement organisé de nature saisonnière ou cyclique des troupeaux à la recherche d'eau, de pâturages et/ou de cures salées.

- troupeau : ensemble d'animaux de la même espèce, évoluant en groupe dans un contexte donné. 
- mobilité : fait du déplacement d'un animal ou d'un troupeau d'un point à un autre, à la recherche de ressources pastorales (eau, pâturages, cures salées).

\section{CHAPITRE 3 : PRINCIPES FONDAMENTAUX DU DEVELOPPEMENT PASTORAL DURABLE}

\section{SECTION 1 : ROLE ET RESPONSABILITES DE L'ETAT ET DES COLLECTIVITES TERRITORIALES}

ARTICLE 4: L'Etat burkinabè coopère avec les Etats voisins en vue du développement pastoral durable au plan régional, notamment dans les domaines de la santé animale, de la transhumance, de la commercialisation, de la sécurité des transhumants, de leurs biens et du règlement des litiges.

ARTICLE 5 : L'Etat et les collectivités territoriales garantissent aux pasteurs le droit d'accès aux espaces pastoraux, le droit d'utilisation équitable des ressources naturelles et la mobilité des troupeaux.

L'Etat et les collectivités territoriales prennent également les mesures nécessaires à l'amélioration des performances productives et de qualité de l'élevage pastoral. En particulier, ils créent les conditions de sécurisation des activités pastorales et de préservation de la santé animale ; ils encouragent et soutiennent les activités d'amélioration de l'alimentation des animaux.

ARTICLE 6 : Dans le cadre de la politique d'aménagement du territoire, l'Etat et les collectivités territoriales veillent à l'identification, à la protection et à l'aménagement des espaces à vocation pastorale.

En particulier dans le cadre de l'aménagement des espaces périurbains, l'Etat et les collectivités territoriales réservent les espaces nécessaires à la poursuite et au développement des activités d'élevage.

L'Etat et les collectivités territoriales veillent également à la préservation et à la protection des espaces pastoraux traditionnels. En particulier, ils favorisent dans les régions à vocation pastorale, la préservation de la vocation pastorale des bas-fonds et des zones de bourgou.

ARTICLE 7 : En cas de situation de crise alimentaire du bétail dûment constatée par le gouvernement, le conseil des ministres peut par décret, autoriser l'ouverture temporaire de certaines forêts classées à la pâture des animaux. Le décret autorisant l'ouverture exceptionnelle de chaque forêt précise les restrictions particulières applicables à ladite forêt.

Un arrêté conjoint des ministres chargés de l'élevage, des forêts, de l'eau, de l'aménagement du territoire et de l'administration du territoire précise les modalités d'accès aux forêts classées ouvertes à la pâture en cas de crise alimentaire du bétail.

ARTICLE 8 : Le ministre chargé de l'élevage prend en collaboration avec les ministres concernés, toutes les mesures de nature à encourager et à faciliter l'organisation, l'information et la formation des pasteurs.

En outre, il prend toutes mesures appropriées de nature à promouvoir les échanges et la circulation de l'information pour la bonne conduite des actions et programmes de développement de l'élevage.

96 Textes et textes de loi sur la gestion des ressources naturelles au Burkina Faso 


\section{SECTION 2 : ROLE ET RESPONSABILITES DES PASTEURS}

ARTICLE 9 : Les pasteurs exploitent les ressources naturelles dans le respect des lois et règlements en vigueur, notamment ceux relatifs à l'environnement et à la garantie des biens d'autrui. En concertation avec l'Etat et les collectivités territoriales, leurs organisations participent à la gestion durable des ressources pastorales et à la sauvegarde de l'environnement.

ARTICLE 10 : Les espaces pastoraux et les ressources naturelles font l'objet d'une utilisation partagée et équitable par les différentes catégories d'exploitants ruraux dans le respect des lois et règlements en vigueur. Chaque utilisateur est tenu de respecter les droits légitimes des autres utilisateurs.

ARTICLE 11: Les organisations de pasteurs, en concertation avec les autres organisations de producteurs et les autorités coutumières, doivent apporter leur concours aux actions d'identification, de préservation et de gestion des espaces pastoraux, des points d'eau et des pistes à bétail. Ils contribuent également dans les mêmes conditions aux niveaux national et local, aux actions de prévention et de résolution des litiges liés aux activités pastorales.

ARTICLE 12: Les organisations de pasteurs prennent toutes les mesures utiles en vue de l'information de leurs membres sur la législation et la politique de développement de l'élevage. Conformément à la législation en vigueur relative au régime associatif, elles sont tenues de rendre compte à leurs membres des résultats des concertations avec les autorités publiques.

\section{TITRE II : EXPLOITATION DES RESSOURCES NATURELLES AUX FINS DE PASTORALISME}

CHAPITRE 1 : ACCES AUX RESSOURCES PASTORALES

\section{SECTION 1 : ESPACES AFFECTES A LA PATURE}

ARTICLE 13 : Dans les espaces pastoraux d'aménagement spécial, ont accès aux ressources pastorales les pasteurs bénéficiaires d'autorisation ou de titre d'installation.

ARTICLE 14 : Dans les espaces de terroir réservés à la pâture, les pasteurs accèdent librement aux ressources pastorales. Cependant, les communautés de base en collaboration avec les organisations d'éleveurs concernés, peuvent être autorisées, en vue d'une gestion durable des ressources naturelles, à prévoir des mesures locales d'accès à ces ressources.

ARTICLE 15 : Les conditions dans lesquelles les communautés de base assurent la gestion locale des ressources pastorales de leur terroir et prévoient les mesures locales d'accès sont précisées par voie d'arrêté conjoint des ministres chargés de l'élevage, de l'eau, des forêts, de l'agriculture, de l'administration du territoire et des finances.

ARTICLE 16 : Les droits collectifs reconnus à l'article 13 ci-dessus aux pasteurs installés dans les espaces pastoraux d'aménagement spécial sont assimilés à des droits réels. Ils portent sur les ressources pastorales et ne préjugent en rien de la propriété du fond de terre.

Les pasteurs ne peuvent être privés des droits qui leur sont ainsi reconnus que pour cause d'utilité publique et sous réserve d'une juste et préalable compensation. 
ARTICLE 17 : Les espaces affectés aux activités pastorales tels que définis à l'article 3 sont incorporés, selon les cas, dans le domaine de l'Etat ou dans celui des collectivités territoriales.

Les collectivités territoriales peuvent créer des espaces pastoraux d'aménagement spécial dans leurs limites territoriales.

ARTICLE 18 : Les espaces pastoraux d'aménagement spécial existant à la date d'entrée en vigueur de la présente loi sont incorporés dans le domaine de l'Etat qui procède à la délimitation, au bornage et à l'immatriculation de ces espaces en son nom.

L'Etat peut en céder la propriété aux collectivités locales territorialement compétentes, à charge pour ces dernières de ne pas changer la destination de l'espace concerné. Il peut aussi par convention, en confier la gestion à ces collectivités territoriales et/ou à des organisations de pasteurs.

ARTICLE 19 : Les espaces pastoraux d'aménagement spécial font l'objet de schémas directeurs d'aménagement élaborés par les services techniques chargés de l'élevage en concertation avec les services techniques partenaires, les organisations d'éleveurs, d'agriculteurs, les structures villageoises et inter-villageoises de gestion des terroirs et les collectivités territoriales concernées.

Le projet de schéma directeur d'aménagement est soumis pour avis aux commissions provinciale, régionale et nationale d'aménagement du territoire. Il est adopté conformément aux dispositions en vigueur relatives aux instruments d'aménagement du territoire.

ARTICLE 20 : Les conditions d'occupation et d'exploitation des espaces pastoraux d'aménagement spécial sont déterminées par un cahier des charges général dont les clauses sont fixées par décret.

Les modalités d'accès aux espaces pastoraux d'aménagement spécial sont déterminées par un cahier des charges spécifique dont les clauses sont fixées selon les cas par arrêté du ministre chargé de l'élevage ou par délibération du conseil de la collectivité territoriale concernée.

ARTICLE 21: L'utilisation des espaces pastoraux d'aménagement spécial donne lieu au paiement d'une redevance fixée selon les cas par délibération du conseil de la collectivité territoriale ou de l'organisation des producteurs concernés.

ARTICLE 22 : Les espaces de terroir réservés à la pâture sont soumis à une gestion concertée entre les organisations de pasteurs et les communautés de base concernées. Les collectivités territoriales apportent leur appui et leur assistance à la gestion de ces espaces.

Les collectivités territoriales et les services techniques apportent leur concours en particulier pour l'identification, le recensement et la délimitation de ces espaces. Ils veillent également, en collaboration avec les organisations des pasteurs et des autres producteurs, à la protection et à l'amélioration de ces espaces. Ils prennent, en outre, toutes mesures nécessaires en vue de garantir l'accès équitable aux ressources pastorales à tous les pasteurs dans le respect des dispositions en vigueur. 
ARTICLE 23 : Sous réserve des prescriptions particulières du schéma directeur d'aménagement ou des décisions valablement prises par les organes locaux de gestion, le défrichement à des fins agricoles de portions de terres des espaces pastoraux d'aménagement spécial et des espaces de terroir réservés à la pâture est interdit.

\section{SECTION 2 : ESPACES OUVERTS A LA PATURE}

ARTICLE 24 : Dans les espaces ouverts à la pâture définis à l'article 3 ci-dessus, les pasteurs exercent leurs droits concurremment avec les autres exploitants ruraux, dans les conditions prévues par la présente loi et les autres législations sectorielles en vigueur.

ARTICLE 25 : Dans les espaces du domaine forestier ouverts à la pâture, les pasteurs bénéficient d'un droit d'usage consistant à y faire paître les animaux. Ce droit d'usage est exercé dans le respect de la législation forestière.

ARTICLE 26 : Sauf restrictions particulières résultant des usages locaux ou de l'interdiction expresse du propriétaire, les terres agricoles laissées en jachère sont accessibles à la pâture des animaux. En cas de contestation, la détermination des usages locaux en la matière est faite par les structures de gestion des terroirs compétentes, en relation avec les responsables locaux représentatifs des réalités socioculturelles.

ARTICLE 27 : Après l'enlèvement des récoltes, les champs sont ouverts aux animaux pour la vaine pâture. Tout exploitant agricole souhaitant conserver les résidus de récoltes est tenu de les enlever ou de les protéger avant la période d'ouverture des champs aux animaux.

Nonobstant les dispositions de l'alinéa ci-dessus, l'accès des animaux à un champ après les récoltes est subordonné à l'accord préalable de son propriétaire.

La période d'ouverture des champs aux animaux est déterminée par un texte pris par les autorités locales territorialement compétentes en concertation avec les organisations de producteurs.

\section{SECTION 3 : ACCES A L'EAU}

ARTICLE 28 : En application des dispositions relatives au régime de l'eau, les pasteurs ont le droit d'accéder aux points d'eau en vue d'abreuver leurs animaux. L'accès à l'eau en vue de l'abreuvement des animaux se fait conformément à la présente loi et à la loi d'orientation relative à la gestion de l'eau.

L'utilisation pastorale des ressources en eau en période de sécheresse se fait dans le respect des mesures prévues par le gouvernement en application des dispositions du régime de l'eau.

ARTICLE 29 : La pollution ou le déversement de produits toxiques dans un point d'abreuvement des animaux est interdit.

ARTICLE 30 : Des servitudes de passage sont imposées aux fonds riverains des points d'eau en vue de permettre l'accès des animaux à l'eau.

La consistance de ces servitudes et les modalités de leur constitution sont déterminées conformément aux dispositions législatives et réglementaires en vigueur. 
ARTICLE 31 : L'établissement des campements et le stationnement des animaux sont interdits aux abords des points d'eau, dans un rayon déterminé par un arrêté conjoint des ministres chargés de l'élevage, des forêts et de l'eau.

ARTICLE 32 : Le défrichement et la mise en culture des terres aux abords immédiats des points d'abreuvement des animaux sont interdits. Un arrêté conjoint des ministres chargés de l'agriculture, de l'élevage, des forêts et de l'eau précise les distances à respecter pour la mise en culture des terres situées à proximité des cours et points d'eau.

ARTICLE 33 : L'accès des animaux aux points d'eau naturels est libre et gratuit. Il peut faire l'objet d'une réglementation par l'autorité compétente notamment en ce qui concerne les modalités de l'abreuvement, du parcage et de la circulation des animaux ou encore, en ce qui concerne les besoins de protection de la qualité des ressources en eau.

ARTICLE 34 : L'accès des animaux aux plans d'eau artificiels, aux puits et aux forages publics est soumis à une réglementation édictée par le ministre chargé de l'eau conjointement avec les ministres chargés de l'élevage, de l'agriculture et de l'énergie. Il peut être subordonné au paiement de redevances.

ARTICLE 35 : L'accès aux puits, forages et plans d'eau privés est soumis à autorisation préalable du propriétaire. Il s'exerce conformément aux conventions librement négociées entre les parties concernées.

\section{CHAPITRE 2 : MOBILITE DES ANIMAUX SECTION 1 : DROITS ET OBLIGATIONS}

ARTICLE 36 : Dans les conditions prévues par la présente loi et les textes en vigueur, le droit de déplacer les troupeaux à des fins pastorales s'exerce sur l'ensemble du territoire national ; il comporte également le droit au franchissement des frontières.

Sous réserve de réciprocité, les troupeaux étrangers sont autorisés à franchir les frontières nationales dans le cadre de la transhumance.

ARTICLE 37 : La garde des animaux est obligatoire en toute saison. L'absence de garde d'animaux constitue une faute, engageant la responsabilité civile du propriétaire des troupeaux pour les dommages éventuels causés aux tiers.

ARTICLE 38 : Tout pasteur transhumant est tenu de se conformer à la législation en vigueur relative à la police sanitaire des animaux, notamment en ce qui concerne les mesures générales de prévention contre les maladies animales contagieuses.

Le pasteur en transhumance est également tenu au respect des dispositions de caractère administratif, notamment en ce qui concerne la détention du certificat de transhumance.

ARTICLE 39 : L'admission de troupeaux étrangers sur le territoire burkinabè est subordonnée à la présentation des documents officiels sanitaires et administratifs relatifs à la transhumance prévus par la législation nationale sur la police zoo-sanitaire et les accords régionaux et internationaux relatifs à la transhumance. Les troupeaux non accompagnés des documents exigés sont mis en quarantaine ou refoulés.

ARTICLE 40 : Les troupeaux en transhumance doivent obligatoirement être accompagnés de gardiens majeurs en nombre suffisant en considération de la taille du

$100 \mid$ Textes et textes de loi sur la gestion des ressources naturelles au Burkina Faso 
troupeau. Les textes d'application de la présente loi précisent les normes applicables en matière de garde des animaux en transhumance en se conformant aux accords sousrégionaux en vigueur en matière de transhumance.

ARTICLE 41 : Les troupeaux en transhumance internationale doivent respecter les postes d'entrée et de sortie prévus par la réglementation en vigueur, ainsi que les périodes d'entrée et de sortie, et les zones d'accueil s'il y a lieu.

ARTICLE 42 : Les collectivités territoriales concernées par la transhumance et les organisations de pasteurs se concertent périodiquement afin de préparer et organiser les mouvements de troupeaux, notamment en ce qui concerne les périodes de départ et de retour.

\section{SECTION 2 : PISTES A BETAIL}

ARTICLE 43 : Le déplacement des animaux se réalise en empruntant les pistes à bétail qui sont de trois catégories :

- les pistes d'accès ;

- les pistes de transhumance;

- les pistes de commercialisation.

Toute mise en culture, construction ou tout établissement d'entrave de quelque nature que ce soit sur l'emprise d'une piste à bétail est interdit.

\section{ARTICLE 44}

Les pistes à bétail sont créées par arrêté conjoint des ministres chargés de l'élevage, de l'agriculture, de l'administration du territoire, de l'eau, des forêts, des infrastructures, des mines et de l'aménagement du territoire après avis de l'autorité locale compétente.

L'arrêté de création fixe l'itinéraire et la délimitation de la piste.

ARTICLE 45 : Les pistes à bétail font l'objet d'un classement selon les cas, dans le domaine public de l'Etat ou des collectivités territoriales. Elles sont de ce fait inaliénables, imprescriptibles et insaisissables.

ARTICLE 46 : Les pistes à bétail doivent être délimitées par tous moyens appropriés. Elles grèvent les fonds riverains d'une servitude d'utilité publique dont l'assiette est déterminée dans les conditions fixées par arrêté conjoint des ministres chargés de l'élevage, de l'agriculture, des forêts, de l'eau, de l'aménagement du territoire et de l'administration du territoire.

Les normes techniques relatives aux pistes à bétail sont précisées par arrêté du ministre chargé de l'élevage.

ARTICLE 47 : En saison de cultures, le déplacement des animaux à des fins pastorales se fait obligatoirement par les pistes à bétail. En dehors de la saison de cultures, l'utilisation de ces pistes est simplement recommandée.

Dans tous les cas, les propriétaires des animaux sont civilement responsables des dommages qu'ils ont, par leur faute, causés aux tiers.

ARTICLE 48 : Pour les transhumances internationales, le respect des pistes de transhumance est obligatoire en toute saison. 


\section{TITRE III : INFRACTIONS, SANCTIONS ET PROCEDURES}

\section{CHAPITRE 1 : INFRACTIONS ET SANCTIONS}

ARTICLE 49 : Quiconque sans autorisation ni titre s'installe, dans les espaces d'aménagement spécial en vue de l'exercice d'activités pastorales est puni d'une amende de 50000 francs à 100000 francs.

ARTICLE 50 : Sauf dispositions contraires des textes législatifs et réglementaires en vigueur et des prescriptions des schémas directeurs d'aménagement, quiconque défriche et/ou met en culture une portion de terre à l'intérieur des limites d'un espace pastoral d'aménagement spécial immatriculé au nom de l'Etat est puni d'une amende de 100000 francs à 300000 francs et d'un emprisonnement de un mois à trois mois, ou de l'une de ces deux peines seulement.

ARTICLE 51 : Sauf dispositions contraires des textes législatifs et réglementaires en vigueur et des prescriptions des schémas directeurs d'aménagement, est puni d'une amende de 50000 francs à 100000 francs et d'un emprisonnement de quinze jours à deux mois ou, de l'une de ces deux peines seulement, celui qui défriche et/ou met en culture une portion de terre à l'intérieur des limites d'un espace pastoral appartenant à une collectivité territoriale.

ARTICLE 52: Sauf autorisation préalable des structures locales de gestion compétentes, celui qui défriche et met en culture un espace délimité de terroir réservé à la pâture est puni d'une amende de 5000 francs à 50000 francs.

ARTICLE 53 : Nonobstant les sanctions encourues aux articles 49, 50, 51 et 52 cidessus le délinquant est tenu de déguerpir immédiatement et de remettre les lieux en l'état à ses frais.

ARTICLE 54 : Sans préjudice de la condamnation au paiement de dommages et intérêts pour les préjudices éventuels causés aux tiers, quiconque procède au déversement dans un point d'abreuvement des animaux, de produits toxiques ayant provoqué ou susceptibles de provoquer leur mort ou des effets nuisibles sur leur santé est puni d'une amende de 50000 francs à 5000000 de francs et d'un emprisonnement de onze jours à deux mois ou de l'une de ces deux peines seulement.

Celui qui procède au déversement sur un pâturage de produits toxiques ayant provoqué ou susceptibles de provoquer la mort ou des effets nuisibles sur la santé des animaux est passible des mêmes peines que celles prévues à l'alinéa précédent.

ARTICLE 55 : Sans préjudice de la condamnation au paiement de dommages et intérêts pour les préjudices causés aux tiers, quiconque accède aux champs avec ses animaux avant la période d'ouverture des champs aux animaux pour la pâture est puni d'une amende de 50000 francs à 500000 francs et d'un emprisonnement de 11 jours à deux mois ou de l'une de ces deux peines seulement.

ARTICLE 56 : Quiconque établit un campement pastoral ou agricole aux abords d'un point d'abreuvement des animaux ou les occupe abusivement est puni d'une amende de 10000 francs à 50000 francs.

La même peine est applicable à celui qui, sauf mesures d'autorisation expresse résultant du schéma directeur d'aménagement, défriche et/ou met en culture des terres aux abords immédiats des points d'abreuvement du bétail. 
ARTICLE 57 : Quiconque conduit ses troupeaux en transhumance sans détention du certificat de transhumance est puni d'une amende de 1000 francs à 5000 francs. L'amende est de 10000 francs à 15000 francs lorsque l'auteur de l'infraction est en transhumance internationale.

ARTICLE 58 : Sans préjudice de la condamnation au paiement de dommages et intérêts pour les préjudices causés aux tiers, quiconque conduit des animaux en transhumance sans disposer de gardiens en nombre suffisant est puni d'une amende de 1000 francs à 15000 francs.

Sans préjudice de la condamnation au paiement de dommages et intérêts pour les dommages causés aux tiers, celui qui abandonne des animaux en déplacement sans surveillance est puni d'une amende de 15000 francs à 50000 francs.

ARTICLE 59 : Quiconque conduit ses troupeaux au franchissement de frontière sans respecter les postes d'entrée et de sortie prévus à cet effet est puni d'une amende de 5 000 francs à 15000 francs.

ARTICLE 60 : Sans préjudice des condamnations au paiement de dommages et intérêts pour dommages causés aux tiers, quiconque en saison pluvieuse n'observe pas le respect des pistes à bétail est puni d'une amende de 1000 francs à 15000 francs.

ARTICLE 61 : Quiconque procède à la réalisation de mise en culture ou de mise en valeur quelconque sur une piste à bétail ou y installe des obstacles de quelque nature que ce soit est puni d'une amende de 5000 francs à 15000 francs, sans préjudice de l'obligation de déguerpissement immédiat à ses frais.

ARTICLE 62 : Sans préjudice de la condamnation à la remise en état des lieux, quiconque procède au déplacement ou à la destruction des bornes, balises et autres moyens de délimitation des espaces pastoraux et des pistes à bétail est puni d'une amende de 5000 francs à 50000 francs.

ARTICLE 63 : En cas de récidive, les peines prévues par la présente loi sont portées au double.

\section{CHAPITRE 2 : PROCEDURES}

\section{SECTION 1 : CONSTATATION DES INFRACTIONS ET POURSUITES}

ARTICLE 64 : Les infractions aux dispositions de la présente loi sont constatées par les officiers de police judiciaire, les agents de police municipale et par les agents assermentés des services de l'Etat.

Les officiers de police judiciaire et les agents assermentés de l'Etat sont habilités à procéder aux enquêtes et à effectuer les confiscations conformément au code de procédure pénale. Ils dressent procès-verbal de leurs constatations.

ARTICLE 65 : Les infractions aux dispositions de la présente loi constituent des contraventions ou des délits et sont punies des sanctions prévues au chapitre $1 \mathrm{du}$ Titre III.

ARTICLE 66 : Les infractions constituant des violations des dispositions législatives et réglementaires en vigueur demeurent punies des sanctions prévues par ces textes. 


\section{SECTION 2 : REGLEMENT DES LITIGES PARAGRAPHE 1 : CONCILIATION}

ARTICLE 67 : En cas de litige, les dégâts causés aux champs et les sévices subis par les animaux font l'objet d'un constat en présence des parties en conflit ou de leurs représentants. Le constat est sanctionné par un procès-verbal.

Avant toute procédure contentieuse, le règlement des litiges liés aux activités pastorales est soumis à une procédure obligatoire de conciliation. Cette procédure se déroule devant une commission locale de conciliation, à laquelle participent des représentants des agriculteurs et des pasteurs.

ARTICLE 68 : La procédure de la conciliation doit impliquer, à toutes les étapes de sa mise en œuvre, les parties en conflit ou leurs représentants.

Le résultat de la procédure de conciliation est constaté par un procès-verbal de conciliation ou de non-conciliation.

Article 69 : Les modalités de constatation des dégâts causés aux champs et des sévices subis par les animaux, la procédure de la conciliation, les modalités d'établissement et de conservation du procès-verbal sont déterminées par décret pris en Conseil des ministres.

ARTICLE 70 : En cas de conciliation, il est mis fin définitivement au litige. Le procèsverbal mentionne la renonciation des parties à s'adresser aux tribunaux pour la même affaire.

ARTICLE 71 : La procédure de conciliation relative aux litiges liés aux activités pastorales est précisée par voie d'arrêté conjoint des ministres chargés de l'élevage, de l'agriculture, de l'eau, des forêts, de l'administration du territoire et de la justice.

Paragraphe 2 : Règlement contentieux

ARTICLE 72 : En cas de non-conciliation, les litiges liés aux activités pastorales sont portés devant les juridictions de droit commun, conformément aux textes en vigueur.

\section{TITRE IV : DISPOSITIONS TRANSITOIRES ET FINALES}

ARTICLE 73 : Des décrets pris en Conseil des ministres, sur rapport conjoint des ministres chargés de l'élevage, de l'agriculture, des forêts, de l'eau et de l'administration du territoire déterminent:

- les modalités del'identification et dela sécurisation des espaces pastoraux d'aménagement spécial et des espaces de terroir affectés à la pâture des animaux ;

- les procédures de garantie collective des droits reconnus aux pasteurs sur ces espaces;

- les modalités d'exploitation concurrentielle des ressources naturelles, notamment les ressources en eau, par les différents exploitants ruraux ;

- les modalités de la participation des populations rurales aux différentes actions et procédures résultant de la présente loi ;

- les conditions de l'ouverture temporaire de certaines forêts classées au pâturage en cas de crise alimentaire du bétail dûment constatée. 
ARTICLE 74 : Les collectivités territoriales en concertation avec les responsables des communautés de base, les responsables d'organisations d'agriculteurs et de pasteurs et les responsables des services techniques déconcentrés procèdent dans la mesure du possible, à l'identification provisoire d'espaces de terroir réservés au repli et au cantonnement des animaux pendant la saison pluvieuse. Ils assistent les utilisateurs dans la mise en ouvre de mécanismes locaux et conventionnels de gestion de ces espaces par les organisations de pasteurs.

ARTICLE 75 : Les pistes à bétail actuellement utilisées par les éleveurs continuent de l'être. En concertation avec l'ensemble des acteurs locaux concernés, les collectivités territoriales prennent toutes mesures nécessaires pour assurer leur bonne gestion et procèdent progressivement à la confirmation ou à la redéfinition des itinéraires des différentes pistes.

ARTICLE 76 : La présente loi sera exécutée comme loi de l'Etat.

Ainsi fait et délibéré en séance publique à Ouagadougou, le 14 novembre 2002

Le Président

Roch Marc Christian KABORE

Le Secrétaire de séance

Tenrebsom Boniface ZANGO 


\section{Annexe VII}

\section{JO SPECIAL N02 DU 20 AVRIL 2005 \\ LOI Nº55-2004/AN PORTANT CODE GENERAL DES COLLECTIVITES TERRITORIALES}

\section{L'ASSEMBLEE NATIONALE}

VU la Constitution ;

VU la résolution $n^{\circ} 001-2002 / A N$ du 05 juin 2002 portant validation du mandat des députés ;

a délibéré en sa séance du 21 décembre 2004

et adopté la loi dont la teneur suit :

\section{DISPOSITIONS PRELIMINAIRES}

ARTICLE 1 : La présente loi établit le code général des collectivités territoriales au Burkina Faso.

Le code général des collectivités territoriales détermine l'orientation de la décentralisation, les compétences et moyens d'action, les organes et l'administration des collectivités territoriales.

ARTICLE 2 : La décentralisation consacre le droit des collectivités territoriales à s'administrer librement et à gérer des affaires propres en vue de promouvoir le développement à la base et de renforcer la gouvernance locale.

ARTICLE 3 : La décentralisation est accompagnée d'une déconcentration des services de l'Etat dans le but de renforcer les capacités d'action des collectivités territoriales.

ARTICLE 4 : La représentation de l'Etat sur le territoire national s'effectue à travers des circonscriptions administratives.

Les circonscriptions administratives sont créées et organisées par décret. Le territoire d'une collectivité territoriale et celui d'une circonscription administrative peuvent coïncider.

La création de la collectivité territoriale n'entraîne pas automatiquement création de la circonscription administrative

ARTICLE 5 : La mise en ouvre de la décentralisation se fait selon la règle de la progressivité et le principe de subsidiarité.

ARTICLE 6 : L'Etat est garant de la solidarité nationale. A ce titre, il organise la solidarité avec les collectivités territoriales et entre les collectivités territoriales.

LIVRE I : DE L'ORIENTATION DE LA DECENTRALISATION TITRE I : PRINCIPES GENERAUX DE L'ORGANISATION DU TERRITOIRE CHAPITRE I : DE L'ORGANISATION DU TERRITOIRE

ARTICLE 7 : Le Burkina Faso est organisé en collectivités territoriales. CHAPITRE II : DES COLLECTIVITES TERRITORIALES

ARTICLE 8 : La collectivité territoriale est une subdivision du territoire dotée de la personnalité juridique et de l'autonomie financière.

106 Textes et textes de loi sur la gestion des ressources naturelles au Burkina Faso 
Elle constitue une entité d'organisation et de coordination du développement.

Les collectivités territoriales sont : la région et la commune.

ARTICLE 9: La collectivité territoriale peut :

1) entreprendre toute action en vue de promouvoir le développement économique, social, culturel, environnemental et participer à l'aménagement du territoire ;

2) passer des contrats avec toutes personnes physiques ou morales, privées ou publiques, dont l'Etat, les autres collectivités territoriales et les établissements publics ou établir des rapports de coopération avec des organisations extérieures au Burkina Faso dans le respect de la souveraineté et des intérêts de la nation ;

3) entreprendre dans les conditions prévues par la loi et dans le cadre de leurs compétences propres, des actions de coopération qui donnent lieu à des conventions avec des collectivités territoriales de pays étrangers ou organismes internationaux publics ou privés oeuvrant dans le domaine du développement ;

4) créer ou acquérir des établissements dans les domaines de l'enseignement, de la santé, de l'environnement ou dans tout autre domaine socio-économique ou culturel;

5) créer des établissements publics locaux pour la gestion d'activités socio-économiques ou culturelles;

6) acquérir des actions ou obligations dans des sociétés ayant pour objet l'exploitation de services locaux ou de services nationaux ouverts à la participation des collectivités territoriales.

ARTICLE 10 : La collectivité territoriale est civilement responsable des dommages qu'elle cause. Le régime de la responsabilité civile de la collectivité territoriale et la compétence des tribunaux pour en connaître sont régis par les dispositions applicables à la responsabilité civile de l'Etat.

ARTICLE 11: Les habitants de la collectivité territoriale ont droit à l'information sur la gestion des affaires locales. Ce droit s'exerce par :

1) une animation de débats publics sur les projets et programmes locaux de développement et sur les grandes orientations du budget local ;

2) la mise à la disposition des personnes physiques ou morales du budget et des comptes des collectivités territoriales ;

3) l'accès du public aux séances des conseils des collectivités territoriales, à l'exception de celles tenues à huis clos ;

4) la publication des délibérations du conseil et des actes des autorités locales relatives au budget, à la création des établissements publics locaux, aux emprunts, à la coopération décentralisée, aux accords passés avec l'Etat ou avec des partenaires extérieurs, à l'acceptation des dons et legs et à la prise de participation dans toute société ; toute personne peut obtenir à ses frais, copies desdits documents auprès du président du conseil de la collectivité ou de tout service public habilité.

Le droit à l'information des habitants sur les affaires locales s'exerce sous réserve des dispositions en vigueur relatives notamment à la publicité des actes des autorités locales et à la liberté d'accès aux documents administratifs.

ARTICLE 12 : Le conseil de la collectivité territoriale peut créer des organes de concertation sur toute question d'intérêt local. Ces organes de concertation 
comprennent des personnes qui peuvent ne pas appartenir au conseil, notamment des représentants des associations locales, des notabilités locales, des personnalités compétentes dans les domaines traités.

Le conseil fixe les missions et la composition de ces organes sur proposition du président du conseil régional ou municipal.

Ces organes ont un rôle consultatif.

ARTICLE 13 :La création, la dénomination, la détermination du chef-lieu, la fixation des limites territoriales, la suppression, la fusion ou la scission, l'organisation et le fonctionnement de la collectivité territoriale, ainsi que le régime électoral applicable, sont déterminés par la loi.

ARTICLE 14: Les collectivités territoriales s'administrent librement dans le respect strict de l'intégrité territoriale et de l'unité nationale, de l'identité et de l'autonomie de chaque collectivité. Les collectivités territoriales sont dirigées par des conseils élus qui règlent les affaires locales par des délibérations.

\section{SECTION 1 : DE LA REGION}

ARTICLE 15: La région a vocation à être un espace économique et un cadre d'aménagement, de planification et de coordination du développement.

ARTICLE 16: Le ressort territorial de la région est constitué par l'ensemble des territoires des communes qui la composent.

L’organe délibérant de la région est le conseil régional.

La région est administrée par le président du conseil régional. Le président est assisté de deux vices-présidents.

\section{SECTION 2 : DE LA COMMUNE}

ARTICLE 17 : La commune est la collectivité territoriale de base.

Le territoire de la commune est organisé en secteurs et/ou en villages.

L'organe délibérant de la commune est le conseil municipal.

La commune est administrée par un maire. Le maire est assisté de deux adjoints.

ARTICLE 18: Il est institué deux types de communes :

- la commune urbaine ;

- la commune rurale.

\section{PARAGRAPHE 1 : DE LA COMMUNE URBAINE}

ARTICLE 19: La commune urbaine est une entité territoriale comprenant au moins une agglomération permanente de vingt cinq mille habitants et dont les activités économiques permettent de générer des ressources budgétaires propres annuelles d'au moins vingt cinq millions (25.000.000) de francs CFA.

ARTICLE 20 : Le territoire de la commune urbaine est organisé en secteurs et le cas échéant en villages.

ARTICLE 21 : Nonobstant les dispositions des articles 19 et 20 ci-dessus, les communes urbaines existantes à la date d'entrée en vigueur de la présente loi conservent leur statut.

Leurs ressorts territoriaux sont étendus à ceux des départements dans lesquels elles sont situées.

108 Textes et textes de loi sur la gestion des ressources naturelles au Burkina Faso 
ARTICLE 22 : L'aménagement du territoire de la commune urbaine est soumis aux dispositions législatives et réglementaires en matière d'aménagement et d'urbanisme et aux dispositions législatives et réglementaires en matière d'aménagement rural du domaine foncier national.

ARTICLE 23 : La commune urbaine peut être érigée en commune à statut particulier, lorsque l'agglomération principale a une population d'au moins deux cent mille habitants et des activités économiques permettant de générer des ressources budgétaires propres annuelles d'au moins cinq cent millions (500.000.000) de francs CFA.

ARTICLE 24 : Les communes urbaines à statut particulier sont organisées en arrondissements regroupant plusieurs secteurs, le cas échéant des villages.

ARTICLE 25 : Les communes urbaines à statut particulier sont soumises aux règles générales applicables aux communes, sous réserve des dispositions de la présente loi et des autres dispositions législatives qui leur sont propres.

ARTICLE 26 : L'arrondissement est un démembrement de la commune à statut particulier.

\section{PARAGRAPHE 2 : DE LA COMMUNE RURALE}

ARTICLE 27 : La commune rurale est un regroupement de villages qui a une population d'au moins cinq mille habitants et dont les activités économiques permettent de générer des ressources budgétaires propres annuelles d'au moins cinq millions (5.000.000) de francs CFA.

Nonobstant les dispositions ci-dessus, les territoires des départements existants à la date d'entrée en vigueur de la présente loi sont érigés en communes rurales.

ARTICLE 28: Le territoire de la commune rurale comprend un espace d'habitation, un espace de production et un espace de conservation.

ARTICLE 29 : L'espace d'habitation est un établissement humain permanent destiné principalement à l'habitat, au commerce, à l'industrie, à l'artisanat, à l'installation des services publics.

ARTICLE 30 : Les espaces de production sont destinés principalement à l'agriculture, à l'élevage, à la foresterie, à la pisciculture et plus généralement à toutes les activités liées à la vie rurale.

Les espaces de conservation constituent des zones de protection des ressources naturelles. Ils prennent notamment en compte les aires de protection de la flore et de la faune.

ARTICLE 31: L'aménagement du territoire de la commune rurale est soumis principalement aux dispositions législatives et réglementaires en matière d'aménagement rural du domaine foncier national et accessoirement aux dispositions législatives et réglementaires en matière d'aménagement et d'urbanisme.

\section{CHAPITRE III : DES PRINCIPES FONDAMENTAUX ET DES MODALITES DE TRANSFERT DE COMPÉTENCES}

ARTICLE 32 : Les collectivités territoriales concourent avec l'Etat, à l'administration et à l'aménagement du territoire, au développement économique, social, éducatif, sanitaire, culturel et scientifique, ainsi qu'à la protection, à la gestion des ressources naturelles et à l'amélioration du cadre de vie. 
ARTICLE 33 : Les compétences des collectivités territoriales sont déterminées par la loi.

ARTICLE 34: La répartition des compétences entre l'Etat et les collectivités territoriales est régie par le principe de subsidiarité.

ARTICLE 35 : La répartition des compétences entre les collectivités territoriales s'effectue, en distinguant celles qui sont dévolues aux régions et aux communes.

La collectivité territoriale régionale reçoit des compétences transversales.

ARTICLE 36 : Les transferts de compétences par l'Etat doivent être accompagnés du transfert aux collectivités territoriales des moyens et des ressources nécessaires à l'exercice normal de ces compétences, dans les conditions prévues par la loi.

ARTICLE 37 :Les ressources nécessaires aux collectivités territoriales pour l'exercice de leurs compétences leur sont dévolues soit par transfert de fiscalité, soit par dotations ou par les deux à la fois.

Toute charge nouvelle incombant aux collectivités territoriales, du fait de la modification par l'Etat des règles relatives à l'exercice des compétences transférées, doit être compensée par un transfert approprié de moyens.

ARTICLE 38 : Les ressources financières des collectivités territoriales sont constituées de recettes propres, de dotations budgétaires de l'Etat et de toutes autres contributions.

ARTICLE 39 : Lorsqu'un groupement de collectivités territoriales exerce des attributions dans un domaine faisant l'objet d'un transfert de compétences, ce transfert s'opère au profit de l'organisme créé à cet effet, sur décision de l'organe délibérant de chaque collectivité territoriale.

ARTICLE 40 : Tout transfert de compétences de l'Etat au profit des régions et des communes s'accompagne du transfert de tout ou partie des services correspondants.

Le transfert de services peut être définitif ou temporaire. Dans ce dernier cas, les conditions d'utilisation de chaque service de l'Etat par les collectivités territoriales sont arrêtées par une convention passée entre le représentant de l'Etat et le président du conseil de la collectivité.

Les conventions sont établies suivant des modèles - types fixés par décret.

ARTICLE 41 : Le transfert d'une compétence entraîne de plein droit, la mise à la disposition de la collectivité territoriale bénéficiaire, des biens meubles et immeubles utilisés à la date de ce transfert, pour l'exercice de cette compétence.

Le transfert est constaté par un décret de dévolution pris en conseil des ministres.

ARTICLE 42 : Les transferts de compétences prévus par la présente loi au profit des régions et des communes ne peuvent autoriser l'une de ces collectivités à établir ou à exercer une tutelle sous quelque forme que ce soit sur l'autre.

ARTICLE 43 : Les collectivités territoriales exercent leurs compétences propres dans le respect des sujétions imposées par la défense nationale.

A ce titre, l'Etat dispose, si nécessaire, des services des régions et des communes, de leurs groupements et de leurs établissements publics.

110 Textes et textes de loi sur la gestion des ressources naturelles au Burkina Faso 


\section{TITRE II DES RAPPORTS ENTRE LETAT ET LES COLLECTIVITES TERRITORIALES}

ARTICLE 44 : L'Etat entretient avec les collectivités territoriales et dans les domaines de leurs compétences, des relations contractuelles, d'assistance et de contrôle.

Les modalités de ces rapports sont précisées par décrets pris en conseil des ministres.

\section{CHAPITRE I : DES RELATIONS CONTRACTUELLES}

ARTICLE 45 : L'Etat et les collectivités territoriales peuvent déterminer par contrats leurs interventions communes dans tous les domaines d'intérêt public national ou local.

ARTICLE 46 : Les collectivités territoriales peuvent passer des contrats entre elles ou avec des personnes physiques ou morales, qu'elles soient privées ou publiques, pour l'exécution de tâches relevant de leurs compétences.

ARTICLE 47 : Les contrats assortis d'obligation de contrepartie de l'Etat sont transmis à l'autorité de tutelle pour autorisation préalable.

\section{CHAPITRE II : DE L'ASSISTANCE DE L'ETAT}

ARTICLE 48 : L'Etat soutient et facilite le développement des collectivités territoriales. Il a envers elles, un devoir d'assistance.

ARTICLE 49 : Le devoir d'assistance de l'Etat vis-à-vis des collectivités territoriales s'exerce notamment sous forme de mise à disposition :

- de subventions ;

- de dotations spéciales ;

- de ressources humaines ou matérielles;

- d'appui technique et financier.

ARTICLE 50 : L'Etat organise avec les collectivités territoriales des mécanismes d'appui technique, financier et de solidarité entre elles.

Les modalités de cet appui sont précisées par décret pris en conseil des ministres.

ARTICLE 51 : Pour accomplir leurs missions, les collectivités territoriales disposent de services propres et peuvent recourir aux services déconcentrés de l'Etat.

ARTICLE 52 : L'autorité de tutelle assure l'appui conseil aux collectivités territoriales. Cet appui conseil fait l'objet d'un rapport dont copie est adressée au président du conseil de la collectivité.

\section{CHAPITRE III : DES CONTROLES DE LETAT}

ARTICLE 53 : Les différents contrôles exercés sur les collectivités territoriales sont :

1) la tutelle ;

2) le contrôle administratif;

3) le contrôle juridictionnel. 


\section{SECTION 1 : DE LA TUTELLE}

ARTICLE 54 : La tutelle administrative et la tutelle financière sont assurées respectivement par le ministre chargé des collectivités territoriales et le ministre chargé des finances qui délèguent par arrêté, une partie de ce pouvoir aux représentants de l'Etat dans les circonscriptions administratives.

Un arrêté conjoint précise les matières faisant l'objet de la délégation.

ARTICLE 55 : Les actes et les délibérations des collectivités territoriales sont soumis au contrôle de légalité exercé par l'autorité de tutelle.

ARTICLE 56 : Le contrôle de tutelle comporte les fonctions :

1) d'approbation ;

2) d'autorisation préalable ;

3) d'annulation ;

4) de suspension ou de révocation ;

5) de substitution ;

6) d'inspection.

ARTICLE 57 : L'approbation et l'autorisation préalable ne concernent que les actes et les délibérations à caractère financier ou ayant une incidence financière, conformément aux dispositions des articles 150 pour ce qui concerne la région et 229 pour ce qui concerne la commune.

ARTICLE 58 : Le délai d'approbation ou d'autorisation préalable des délibérations relatives aux matières visées à l'article 57 ci-dessus est de quarante cinq jours maximum à compter de la date de dépôt auprès de l'autorité compétente.

Passé ce délai, l'approbation ou l'autorisation préalable est réputée acquise.

ARTICLE 59 : Lorsque l'autorité de tutelle refuse l'approbation ou l'autorisation, le conseil de la collectivité peut se pourvoir en justice, à la condition d'avoir saisi l'autorité de tutelle au préalable. Dans ce cas de saisine, l'autorité de tutelle dispose d'un délai de quarante cinq jours maximum pour réagir.

ARTICLE 60 : Les délibérations qui ne sont pas soumises à approbation ou à autorisation préalable deviennent exécutoires après leur transmission à l'autorité de tutelle, sous réserve du respect des conditions d'entrée en vigueur des actes des autorités locales.

ARTICLE 61 : Sont nuls de plein droit toutes décisions, toutes proclamations et adresses, tous avis qui sortent des attributions des autorités locales, ceux qui sont contraires aux lois ou règlements et ceux qui sont pris par des organes illégalement réunis ou constitués. Cette nullité peut être invoquée ou opposée à tout moment par les parties intéressées. La nullité est constatée par l'autorité de tutelle. Notification est faite par l'autorité de tutelle au président du conseil qui en informe le conseil à sa plus prochaine session.

Le recours gracieux devant l'autorité de tutelle est obligatoire avant l'exercice des recours pour excès de pouvoir devant les juridictions.

Article 62: Lorsque le conseil de la collectivité délibère hors d'une session régulièrement convoquée ou lorsqu'il est illégalement constitué, l'autorité de tutelle prend toutes mesures nécessaires pour mettre immédiatement fin à ladite session.

112 Textes et textes de loi sur la gestion des ressources naturelles au Burkina Faso 
Le cas échéant, l'autorité de tutelle constate l'illégalité des actes découlant de cette session.

ARTICLE 63 : L'autorité de tutelle peut suspendre ou annuler soit d'office, soit à la demande de toute personne ayant intérêt, les délibérations et décisions présentant un intérêt personnel pour l'une ou l'autre des autorités ayant participé à la décision ou au règlement, en personne, ou par mandataire.

Dans ce dernier cas, il est accusé réception de la demande en annulation.

La suspension ne peut excéder quarante cinq jours.

ARTICLE 64: La demande en annulation doit être déposée sous peine de forclusion dans un délai de quarante cinq jours à partir de la date de publication ou de notification de l'acte.

L'autorité de tutelle statue dans le délai d'un mois.

ARTICLE 65: L'annulation d'office doit intervenir dans les quarante cinq jours à partir de la date de l'accusé de réception.

La suspension ou l'annulation est notifiée au président du conseil de la collectivité, lequel en informe le conseil à sa plus prochaine session.

ARTICLE 66 : En cas d'inexécution par les autorités de la collectivité territoriale des mesures prescrites par les lois et règlements ou en vertu de ceux-ci, l'autorité de tutelle peut, après une mise en demeure, se substituer à celles-ci et prendre à cette fin toutes mesures utiles.

ARTICLE 67 : Le conseil de la collectivité ou toute partie intéressée peut déposer un recours en annulation pour excès de pouvoir devant les juridictions administratives contre toute décision de l'autorité de tutelle.

ARTICLE 68 : L'autorité de tutelle rapprochée procède à une inspection périodique des collectivités territoriales. Cette inspection fait l'objet d'un rapport dont copie est adressée au président du conseil de la collectivité qui en informe le conseil à sa plus prochaine session.

\section{SECTION 2 : DU CONTROLE ADMINISTRATIF}

ARTICLE 69 : Les collectivités territoriales sont soumises au contrôle des différents corps de contrôle de l'Etat conformément aux textes en vigueur.

\section{SECTION 3 : DU CONTROLE JURIDICTIONNEL}

ARTICLE 70: Le juge administratif est juge du contentieux né de l'exercice du contrôle de légalité.

ARTICLE 71 : Les comptes des collectivités territoriales et de leurs établissements publics sont soumis au contrôle de la Cour des comptes.

\section{TITRE III : DE LA MISE EN CEUVRE DE LA DECENTRALISATION}

CHAPITRE I : DE LA MISE EN PLACE DES ORGANES ET DU TRANSFERT DES COMPETENCES DE L'ETAT AUX COLLECTIVITES TERRITORIALES

ARTICLE 72 : Le transfert des compétences et des ressources de l'Etat aux collectivités territoriales s'effectue selon la règle de la progressivité.

\section{SECTION 1 : DE LA MISE EN PLACE DES ORGANES}

ARTICLE 73 : La mise en place des organes délibérants dans les régions et les communes doit être effective au plus tard en 2007. 
En attendant la mise en place effective des conseils régionaux, les attributions desdits conseils sont exercées par des délégations spéciales régionales.

Les attributions des présidents et des vices-présidents de conseils régionaux sont exercées par les gouverneurs.

En attendant la mise en place des conseils municipaux dans les communes où il n'en existe pas, les attributions desdits conseils sont exercées par des délégations spéciales.

Les attributions des maires et des adjoints sont exercées par les préfets.

Un décret pris en conseil des ministres précise la composition de chaque délégation spéciale.

ARTICLE 74: Un décret pris en conseil des ministres détermine les échéances effectives de mise en place du fichier électoral et les dates des différents scrutins locaux.

\section{SECTION 2 : DU TRANSFERT DES COMPETENCES DE LETAT AUX COLLECTIVITES TERRITORIALES}

ARTICLE 75 :Le transfert des compétences à la région doit être effectif trois ans au plus tard après la mise en place des organes délibérants.

ARTICLE 76 : Le transfert des compétences aux communes urbaines dans les domaines de la santé, du préscolaire, de l'enseignement de base, de l'alphabétisation, de la jeunesse, de la culture et des sports et loisirs doit être effectif au plus tard en 2005.

ARTICLE 77 : Concernant les communes rurales, le transfert des compétences et des ressources nécessaires à l'exercice des compétences doit être effectif trois ans au plus tard après la mise en place des organes délibérants.

Toutefois, peuvent être transférées dès la mise en place des organes délibérants, les compétences et les ressources dans les domaines de la santé, du préscolaire, de l'enseignement de base, de l'alphabétisation, de la culture, du sport et des loisirs, de l'environnement et de la gestion des ressources naturelles.

\section{CHAPITRE II : DE L'ACCOMPAGNEMENT DE LA DECENTRALISATION}

ARTICLE 78 : Le gouvernement prend toutes les dispositions nécessaires pour rendre effectives au 30 juin 2005 au plus tard:

- les délégations de pouvoirs aux représentants de l'Etat dans les circonscriptions administratives ;

- les délégations de compétences des ministres aux responsables des services techniques déconcentrés.

\section{LIVRE II : DES COMPETENCES ET DES MOYENS D'ACTION DES COLLECTIVITES TERRITORIALES \\ TITRE I DES COMPETENCES DES COLLECTIVITES TERRITORIALES}

ARTICLE 79 : Les collectivités territoriales concourent avec l'Etat, à l'administration et à l'aménagement du territoire, au développement économique, social, éducatif, sanitaire, culturel et scientifique, ainsi qu'à la protection, à la mise en valeur des ressources naturelles et à l'amélioration du cadre de vie.

Les collectivités territoriales peuvent se regrouper suivant les intérêts locaux ou l'intérêt général pour réaliser des activités de développement. 


\section{CHAPITRE I : DES DOMAINES DE COMPETENCES DES COLLECTIVITES TERRITORIALES}

\section{Section 1 : Du domaine foncier des collectivités territoriales}

ARTICLE 80 : Les collectivités territoriales disposent d'un domaine foncier propre, constitué par les parties du domaine foncier national cédées à titre de propriété par l'Etat.

ARTICLE 81 : Dans le respect des principes et dispositions de la loi portant réorganisation agraire et foncière, l'Etat peut transférer aux collectivités territoriales la gestion et l'utilisation du domaine foncier national et de son domaine privé situés dans leurs ressorts territoriaux.

La gestion et l'utilisation des parties du domaine foncier transférées aux collectivités territoriales sont soumises à l'autorisation préalable de la tutelle.

ARTICLE 82 : Pour les projets et opérations qu'il initie sur le domaine foncier national, l'Etat prend la décision après consultation de la collectivité sur le territoire de laquelle se situe le projet ou l'opération.

ARTICLE 83 : L'Etat peut céder aux collectivités territoriales, tout ou partie de ses biens meubles ou immeubles, relevant de son domaine privé ou passer avec elles des conventions portant sur l'utilisation desdits biens.

\section{SECTION 2 : DE LAMENAGEMENT DU TERRITOIRE, DE LA GESTION DU DOMAINE FONCIER ET DE L'AMENAGEMENT URBAIN}

ARTICLE 84: L'aménagement et la gestion du domaine foncier transféré sont soumis à l'autorisation préalable de la tutelle.

ARTICLE 85 : La région reçoit les compétences suivantes :

1) compétence partagée avec l'Etat pour l'initiative d'élaboration du schéma régional d'aménagement du territoire, conformément aux procédures prévues par les textes en vigueur ;

2) avis sur le schéma régional d'aménagement du territoire et les schémas directeurs d'aménagement et d'urbanisme réalisés à l'intérieur du territoire régional avant leur approbation par l'Etat ;

3) soutien à la promotion du transport en commun à l'intérieur de la région ;

4) délivrance d'autorisations d'occupation du domaine foncier national géré par la région ;

5) construction et entretien des pistes rurales.

ARTICLE 86: La commune urbaine et la commune rurale reçoivent les compétences suivantes :

1) avis sur leschéma d'aménagement urbain avant son approbation parl'Etatconformément aux procédures prévues par les textes en vigueur ;

2) établissement et exécution de plans de lotissement, après approbation de l'autorité de tutelle conformément aux textes en vigueur ;

3) attribution des parcelles et délivrance des titres d'occupation se rapportant à leur domaine foncier propre ou aux parties du domaine foncier national ayant fait l'objet d'un transfert de gestion à leur profit ; 
4) délivrance des autorisations de construire ;

5) délivrance des certificats de conformité ;

6) adressage et dénomination des rues ;

7) participation à la gestion des terres du domaine foncier national situé dans leur ressort territorial ;

8) délivrance des autorisations d'occupation du domaine public ;

9) réglementation et police de la circulation ;

10) création, réhabilitation et entretien des rues et des signalisations ;

11) désignation des sites des gares et des aires de stationnement ;

12) construction et entretien des caniveaux, des gares et aires de stationnement ;

13) initiative et soutien en matière de transport en commun ;

14) initiative et soutien en matière de ramassage scolaire.

ARTICLE 87 : En outre, la commune rurale reçoit les compétences spécifiques suivantes :

1) avis sur le schéma d'aménagement de l'espace d'habitation avant son approbation par l'Etat, conformément aux procédures prévues par les textes en vigueur ;

2) participation à l'élaboration du schéma d'aménagement de l'espace de production et de conservation ;

3) participation à la construction et à l'entretien des pistes rurales.

\section{SECTION 3 : DE L'ENVIRONNEMENT ET DE LA GESTION DES RESSOURCES NATURELLES}

ARTICLE 88 : La région reçoit les compétences suivantes :

1) création de bois et de forêts d'intérêt régional ;

2) participation à la protection, à la gestion et à la mise en défens des forêts classées et des forêts protégées ;

3) participation à la protection des cours d'eau ;

4) prévention et lutte contre les feux de brousse et contre la coupe abusive du bois dans les bois et forêts d'intérêt régional;

5) protection de la faune et des ressources halieutiques d'intérêt régional ;

6) articipation à la gestion et à l'exploitation des périmètres aquacoles d'intérêts économiques (PAIE) ;

7) élaboration, mise en oeuvre et suivi des plans ou schémas régionaux d'action pour l'environnement ;

8) délivrance des autorisations de coupe de bois dans le domaine foncier national concédé à la région ;

9) participation à l'établissement par les services de l'Etat des schémas directeurs et des plans d'enlèvement et d'élimination des déchets.

ARTICLE 89 : La commune urbaine et la commune rurale reçoivent les compétences suivantes : 
1) élaboration de plans communaux d'action pour l'environnement ;

2) participation à la protection et à la gestion des ressources en eaux souterraines, en eaux de surface et des ressources halieutiques ;

3) assainissement ;

4) lutte contre l'insalubrité, les pollutions et les nuisances diverses ;

5) création, réhabilitation et gestion des espaces verts et des parcs communaux ;

6) lutte contre la divagation des animaux et réglementation de l'élevage ;

7) enlèvement et élimination finale des déchets ménagers ;

8) délivrance d'autorisation préalable de coupe de bois à l'intérieur du territoire communal;

9) participation à la conservation et à la gestion de ressources naturelles renouvelables d'intérêt régional ou national ;

10) prévention et lutte contre les feux de brousse et contre la coupe abusive du bois ;

11) participation à la protection et à la gestion des ressources fauniques des forêts classées;

12) protection et gestion des ressources fauniques des forêts protégées ;

13) avis sur l'installation des établissements insalubres, dangereux et incommodes de première et deuxième classes conformément au code de l'environnement.

ARTICLE 90: En outre, la commune rurale reçoit les compétences spécifiques suivantes :

1) gestion de la zone de production aménagée par la commune rurale ;

2) participation à la gestion de la zone de production aménagée par d'autres personnes morales, sur le territoire de la commune rurale ;

3) création de zones de conservation ;

4) participation à la protection et à la gestion des ressources naturelles situées sur le territoire de la commune rurale ;

4) participation à la protection et à la gestion des forêts naturelles, de la faune sauvage, des ressources en eau et des ressources halieutiques situées sur le territoire de la communale rurale.

5) participation à la protection et à la gestion des forêts naturelles, de la faune sauvage, des ressources en eau et des ressources halieutiques situées sur le territoire de la commune rurale ;

6) délivrance des autorisations de coupe de bois dans les bois, forêts et zones de conservation d'intérêt local ;

7) délivrance des permis de petite chasse dans les zones de conservation d'intérêt local ;

8) délivrance des permis de pêche sportive sur les cours et plans d'eau d'intérêt local. 


\section{SECTION 4 : DU DEVELOPPEMENT ECONOMIQUE ET DE LA PLANIFICATION}

ARTICLE 91 : Les collectivités territoriales élaborent et exécutent leurs politiques et plans de développement dans le respect des grandes orientations de l'Etat. A cet effet, elles bénéficient de l'appui des services compétents de l'Etat dans les conditions définies par la loi.

L'appui des services de l'Etat a pour objectifs :

1) d'assurer une meilleure conception et mise en œuvre des plans locaux de développement ;

2) de permettre une meilleure harmonisation des différents plans de développement des collectivités territoriales et leur cohérence avec le plan national de développement économique et social ;

3) de favoriser la constitution et la conservation des banques de données nécessaires à toute planification ;

4) d'apporter un appui aux financements des projets productifs, notamment ceux au profit des franges déshéritées de la population.

ARTICLE 92: Les collectivités territoriales peuvent passer avec l'Etat ou avec d'autres personnes morales, des contrats - plans ou des contrats - programmes pour la réalisation d'objectifs de développement économique, social, culturel, sanitaire, scientifique et environnemental.

\section{SECTION 5 : DE LA SANTE ET DE L'HYGIENE}

ARTICLE 93 : La région reçoit les compétences suivantes :

1) participation à la construction et à la gestion des formations sanitaires de base;

2) construction et gestion des formations sanitaires intermédiaires ;

3) organisation de l'approvisionnement pharmaceutique ;

4) réglementation et prise de mesures relatives à l'hygiène, à la salubrité et à la prévention des maladies;

5) valorisation de la pharmacopée traditionnelle ;

6) participation à la résolution des problèmes de santé ;

7) participation à l'établissement de la tranche régionale de la carte sanitaire nationale.

ARTICLE 94 : La commune urbaine et la commune rurale reçoivent les compétences suivantes :

1) construction et gestion des formations sanitaires de base;

2) organisation de l'approvisionnement pharmaceutique et prise de mesures relatives à la réglementation et à la prévention des maladies;

3) prise de mesures d'hygiène et de salubrité dans leur ressort territorial ;

4) contrôle de l'application des règlements sanitaires ;

5) participation à la résolution des problèmes de santé;

6) participation à l'établissement de la tranche communale de la carte sanitaire nationale.

118 Textes et textes de loi sur la gestion des ressources naturelles au Burkina Faso 


\section{SECTION 6 : DE L'EDUCATION, DE LEMPLOI, DE LA FORMATION PROFESSIONNELLE ET DE L'ALPHABETISATION}

ARTICLE 95 : La région reçoit les compétences suivantes :

1) participation à la prise en charge de l'enseignement préscolaire ;

2) participation à la prise en charge du développement de l'enseignement primaire ;

3) participation à la construction et à la gestion des établissements secondaires ;

4) prise en charge, avec l'appui de l'Etat, du développement de l'enseignement supérieur;

5) prise en charge, avec l'appui de l'Etat, de la promotion de l'emploi, de la formation professionnelle et de l'alphabétisation ;

6) participation à l'établissement de la tranche régionale de la carte éducative nationale.

ARTICLE 96 : La commune urbaine et la commune rurale reçoivent les compétences suivantes :

1) prise en charge du développement de l'enseignement préscolaire: acquisition, construction et gestion des établissements préscolaires ;

2) prise en charge du développement de l'enseignement primaire dans le territoire communal : construction ou acquisition et gestion des écoles primaires ;

3) contribution au développement de l'enseignement secondaire: acquisition, construction et gestion des établissements secondaires ;

4) prise en charge avec l'appui de l'Etat, du développement de la formation professionnelle et de l'alphabétisation ;

5) participation à l'établissement de la tranche communale de la carte éducative nationale.

\section{SECTION 7 : DE LA CULTURE, DES SPORTS ET DES LOISIRS}

ARTICLE 97 : La région reçoit les compétences suivantes :

1) construction et gestion des infrastructures culturelles, sportives et de jeunesse ;

2) promotion d'activités culturelles, sportives et de jeunesse ;

3) construction et gestion de musées et bibliothèques régionaux;

4) promotion du tourisme et de l'artisanat ;

5) valorisation des potentiels culturels et artistiques traditionnels de la région;

6) gestion et conservation des archives régionales.

ARTICLE 98 : La commune urbaine et la commune rurale reçoivent les compétences suivantes :

1) construction et gestion des infrastructures sociales, culturelles, sportives et de jeunesse;

2) promotion d'activités culturelles, sportives et de jeunesse ;

3) construction et gestion de musées et bibliothèques communaux ;

4) promotion du tourisme et de l'artisanat ;

5) valorisation des potentiels culturels et artistiques traditionnels de la commune ;

6) gestion et conservation des archives communales ;

7) création et gestion des sites et monuments ;

8) suivi de la restauration et de la réhabilitation des sites et monuments historiques. 


\section{SECTION 8 : DE LA PROTECTION CIVILE, DE LASSISTANCE ET DES SECOURS}

ARTICLE 99 : La région reçoit les compétences suivantes :

1) participation à la protection et à la promotion des droits humains ;

2) participation à la promotion et à la protection sociale des individus et des groupes;

3) contribution à l'organisation et à la gestion des secours au profit des groupes vulnérables et des sinistrés ;

4) participation à l'organisation de la protection civile et de la lutte contre l'incendie ; à ce titre elle peut créer et gérer des unités de sapeurs-pompiers;

5) participation à la lutte contre les autres calamités.

ARTICLE 100 : La commune urbaine et la commune rurale reçoivent les compétences suivantes :

1) participation à la protection et à la promotion des droits humains ;

2) participation à la promotion sociale des individus et des groupes ;

3) participation à l'organisation et à la gestion des secours au profit des groupes vulnérables et des sinistrés ;

4) participation à l'organisation de la protection civile et de la lutte contre l'incendie ; à ce titre, elles peuvent créer et gérer des unités de sapeurs-pompiers.

\section{SECTION 9 : DES POMPES FUNEBRES ET DES CIMETIERES}

ARTICLE 101 : La commune urbaine et la commune rurale reçoivent les compétences suivantes :

1) pouvoir de réglementation en matière de pompes funèbres et de cimetières dans le territoire communal ;

2) aménagement et gestion des cimetières ;

3) délivrance des permis d'inhumer ou d'autorisations de transfert des restes mortels ;

4) contrôle du respect de la réglementation en matière d'opérations funéraires et de transfert des restes mortels ;

5) création et gestion des pompes funèbres ;

6) construction, entretien et gestion des morgues.

\section{SECTION 10 : DE L'EAU ET DE L'ELECTRICITE}

ARTICLE 102 : La région reçoit les compétences suivantes :

1) avis sur les programmes nationaux d'approvisionnement en eau ;

2) participation à l'élaboration du schéma directeur régional d'approvisionnement en eau ;

3) participation à l'entretien et à la conservation des cours d'eau ;

4) participation à la réalisation et à l'entretien des retenues, des barrages, des puits et forages ;

5) avis sur les plans d'électrification dans la région ;

6) participation à l'élaboration du schéma directeur régional d'électrification ;

7) participation à l'élaboration du schéma national d'électrification.

120 Textes et textes de loi sur la gestion des ressources naturelles au Burkina Faso 
ARTICLE 103 : La commune urbaine et la commune rurale reçoivent les compétences suivantes:

1) avis sur le schéma directeur d'approvisionnement en d'eau;

2) élaboration et mise en ouvre des plans locaux de production, de distribution et de maitrise d'énergie ;

3) création et gestion des infrastructures énergétiques ;

4) production et distribution de l'eau potable ;

5) réalisation et gestion de puits, de forages et de bornes-fontaines ;

6) participation à l'élaboration du schéma régional d'électrification ;

7) réalisation et gestion de l'éclairage public.

\section{SECTION 11 : DES MARCHES, ABATTOIRS ET FOIRES}

ARTICLE 104 : La région reçoit les compétences suivantes :

1) création, aménagement et gestion des aires de foires ;

2) organisation des foires régionales ;

3) participation à la construction et à la gestion des abattoirs régionaux.

ARTICLE 105 : La commune urbaine et la commune rurale reçoivent les compétences suivantes :

- création, aménagement et gestion des marchés ;

- construction et gestion des abattoirs et aires d'abattage.

\section{TITRE II DES MOYENS FINANCIERS DES COLLECTIVITES TERRITORIALES}

\section{CHAPITRE I : PRINCIPES GÉNÉRAUX}

ARTICLE 106 : La collectivité territoriale dispose d'un budget propre.

ARTICLE 107 : La nomenclature et les modalités de présentation du budget sont déterminées par décret pris en conseil des ministres, sur proposition du ministre chargé des finances.

ARTICLE 108: Le régime financier et comptable des collectivités territoriales est précisé par décret pris en conseil des ministres, sur proposition des ministres de tutelle.

ARTICLE 109 : Les ressources et les charges des collectivités territoriales sont déterminées par la loi.

ARTICLE 110 : Les ressources nécessaires à l'exercice des missions des collectivités territoriales leur sont dévolues soit par transfert de fiscalité, soit par dotations, soit par les deux à la fois.

ARTICLE 111 : Le budget de chaque collectivité territoriale prévoit pour une année financière, toutes les recettes et les dépenses de la collectivité, sans contraction entre les unes et les autres.

Il est la traduction financière de la tranche annuelle du programme d'action et de développement de la collectivité territoriale.

ARTICLE 112 : Le budget est proposé par l'organe exécutif de la collectivité territoriale, voté par le conseil et approuvé par l'autorité de tutelle dans les conditions fixées par la loi et dans le respect des règles de la comptabilité publique. 
ARTICLE 113 : Le budget de la collectivité territoriale est établi en section ordinaire ou de fonctionnement et en section extraordinaire ou d'investissement.

A la section ordinaire ou de fonctionnement correspondent les recettes et les dépenses ordinaires ou de fonctionnement.

A la section extraordinaire ou d'investissement correspondent les recettes et les dépenses extraordinaires ou d'investissement.

\section{CHAPITRE II : DES RECETTES DES COLLECTIVITES TERRITORIALES}

ARTICLE 114 : Les recettes des collectivités territoriales comprennent des recettes ordinaires ou de fonctionnement et des recettes extraordinaires ou d'investissement.

ARTICLE 115 : Les recettes ordinaires des collectivités territoriales proviennent du produit des recettes fiscales, de l'exploitation du domaine foncier et des services locaux, des subventions, des ristournes accordées par l'Etat ou d'autres collectivités publiques sur le montant des impôts et taxes recouvrés à leur profit, des recettes temporaires ou accidentelles et de la répartition annuelle des fonds de dotation des collectivités territoriales.

ARTICLE 116 : Les collectivités territoriales disposent d'une compétence générale en matière de détermination des tarifs ou taxes rémunératoires du domaine et des services locaux dans les conditions prévues par les lois et règlements en vigueur.

ARTICLE 117 : Les fonds de dotation des collectivités territoriales prévus par la présente loi reçoivent un montant déterminé lors de l'adoption des lois de finances annuelles.

Les fonds de dotation sont gérés conformément aux dispositions du régime financier des collectivités territoriales.

Les modalités de répartition des fonds de dotation sont fixées chaque année par arrêté conjoint du ministre chargé des collectivités territoriales et du ministre chargé des finances.

ARTICLE 118 : Les recettes d'investissement comprennent la part des fonds de dotation, les fonds d'emprunts, les recettes temporaires ou accidentelles, les crédits alloués par le budget de l'Etat ou par tout autre organisme public ou privé, sous forme de fonds de concours et les prélèvements effectués au profit de la section d'investissement à partir de la section de fonctionnement.

ARTICLE 119 : Les collectivités territoriales peuvent recourir à l'emprunt pour la réalisation de leurs investissements de développement.

Ces emprunts peuvent bénéficier de la garantie de l'Etat.

\section{CHAPITRE III: DES DEPENSES DES COLLECTIVITES TERRITORIALES}

ARTICLE 120 : Les dépenses des collectivités territoriales comprennent des dépenses ordinaires ou de fonctionnement et des dépenses extraordinaires ou d'investissement.

Les dépenses de fonctionnement ont un caractère permanent et permettent à la collectivité territoriale de faire face à ses charges et obligations courantes.

Les dépenses d'investissement permettent la réalisation des équipements, bâtiments et autres infrastructures, ainsi que l'acquisition de matériels relatifs à ces travaux.

ARTICLE 121: Les dépenses des collectivités territoriales sont obligatoires ou facultatives.

122 Textes et textes de loi sur la gestion des ressources naturelles au Burkina Faso 
Sont obligatoires pour les collectivités territoriales les dépenses mises à leur charge par la loi.

Les dépenses obligatoires doivent nécessairement figurer au budget. Elles doivent faire l'objet d'ouverture de crédits suffisants avant qu'il ne soit possible à la collectivité d'inscrire des dépenses facultatives.

Une dépense facultative ne peut être inscrite au budget que lorsqu'elle présente un caractère d'intérêt local.

\section{TITRE III : DES RELATIONS ENTRE COLLECTIVITES TERRITORIALES CHAPITRE 1 : DE L'ENTENTE ET DU JUMELAGE}

ARTICLE 122 : Les collectivités territoriales burkinabé peuvent instituer entre elles ou entre elles et des collectivités territoriales étrangères, des relations de coopération.

Cette coopération peut se faire au moyen de l'entente ou du jumelage.

\section{SECTION 1 : DE L'ENTENTE}

ARTICLE 123 : L’entente est l'acte par lequel, des collectivités territoriales burkinabé instituent entre elles, des relations de coopération sur des objets d'utilité publique locale compris dans leurs attributions et les intéressant conjointement.

Les mêmes relations peuvent être instituées entre une ou plusieurs collectivités territoriales nationales et une ou plusieurs collectivités territoriales étrangères.

Ces collectivités territoriales peuvent passer des conventions à l'effet d'entreprendre ou de conserver à frais communs, des ouvrages et des institutions d'utilité publique.

ARTICLE 124 : Le cadre de la coopération instituée par l'entente est la conférence.

La conférence n'est pas dotée de la personnalité juridique et les différentes collectivités parties à l'entente y sont représentées sur une base d'égalité.

ARTICLE 125 : Les décisions des conférences ne sont exécutoires, qu'après autorisation par délibération, de chacune des collectivités territoriales concernées et après approbation de l'autorité de tutelle.

ARTICLE 126 : Les représentants de l'Etat du ressort territorial des collectivités parties à l'entente peuvent assister aux conférences ou s'y faire représenter avec un statut d'observateur.

Les frais occasionnés par leur participation sont à la charge de l'Etat.

\section{SECTION 2 : DU JUMELAGE}

ARTICLE 127: Le jumelage est l'acte solennel par lequel, des collectivités territoriales burkinabé instituent entre elles, ou entre elles et des collectivités territoriales étrangères, des relations de coopération en vue de la réalisation d'un idéal commun, notamment dans les domaines économique, culturel et social.

ARTICLE 128: Le jumelage ne peut s'effectuer que par l'intermédiaire d'un comité de jumelage.

L'organisation et le fonctionnement des comités de jumelage sont fixés par décret pris en conseil des ministres, sur proposition du ministre chargé des collectivités territoriales.

ARTICLE 129 : Les collectivités territoriales peuvent adhérer à des associations nationales ou internationales de jumelage et de coopération inter collectivités. 


\section{CHAPITRE II : DES COMMUNAUTÉS DE COMMUNES}

ARTICLE 130 : Plusieurs communes relevant d'une même région peuvent s'associer pour créer une communauté de communes.

Aucune commune ne peut appartenir à plus d'une communauté de communes.

ARTICLE 131 : La communauté de communes est un établissement public.

Elle est chargée de la gestion d'affaires d'intérêt intercommunal.

Les lois et règlements relatifs à l'administration communale lui sont applicables, en l'absence de dispositions expresses contraires.

ARTICLE 132 : Les communautés de communes sont directement responsables et supportent sur leurs deniers, les dommages causés aux usagers ou aux tiers, pour les faits et actes qui leur sont imputables.

ARTICLE 133 : La communauté de communes est établie sur la base d'une convention approuvée par délibération de chacun des conseils municipaux intéressés.

L'adhésion, le retrait ainsi que l'organisation et le fonctionnement de la communauté de communes sont précisés par décret pris en conseil des ministres.

\section{TITRE IV : DES RELATIONS ENTRE COLLECTIVITES TERRITORIALES ET D'AUTRES PERSONNES MORALES DE DROIT PUBLIC OU PRIVE CHAPITRE I: DES STRUCTURES DE CONCERTATION ET DE COOPÉRATION}

ARTICLE 134: Les collectivités territoriales burkinabé peuvent instituer entre elles et des personnes morales de droit public ou privé, nationales ou étrangères, des structures de concertation et de coopération sur des questions d'intérêt commun.

Lorsqu'un groupement de collectivités territoriales exerce des attributions dans un domaine faisant l'objet d'un transfert de compétences, ce transfert s'opère au profit de cet organisme sur décision des organes délibérants locaux.

Ces structures de concertation et de coopération ne sont pas dotées de la personnalité juridique.

ARTICLE 135 : La création de la structure de concertation et de coopération fait l'objet d'une convention passée entre les parties concernées.

La convention n'est exécutoire, qu'après autorisation par délibération de chacune des collectivités territoriales et après approbation par l'autorité de tutelle.

ARTICLE 136: Les représentants de l'Etat du ressort territorial des collectivités territoriales parties à la convention peuvent assister ou se faire représenter aux réunions de la structure de concertation et de coopération avec un statut d'observateur.

ARTICLE 137: Les décisions prises par la structure de concertation et de coopération ne sont exécutoires, qu'après leur ratification par les instances délibérantes.

\section{CHAPITRE II : DES GROUPEMENTS D’INTÉR T PUBLIC (G.I.P.)}

ARTICLE 138: Des groupements d'intérêt public peuvent être constitués par accord entre des collectivités territoriales, l'Etat, des établissements publics ou toute personne physique ou morale de droit public ou privé, en vue d'une oeuvre ou d'un service présentant une utilité pour chacune des parties.

124 Textes et textes de loi sur la gestion des ressources naturelles au Burkina Faso 
ARTICLE 139: Le groupement d'intérêt public est une personne morale de droit public.

Un décret en fixe les modalités de fonctionnement et détermine les conditions d'exercice du contrôle administratif, technique et financier.

ARTICLE 140 : Le groupement d'intérêt public peut réaliser son objet par voie d'exploitation directe ou par simple participation financière dans des sociétés ou organismes à participation publique majoritaire ou autres organismes dans les mêmes conditions que les collectivités territoriales.

Les modalités de cette participation sont fixées par les actes constitutifs.

\section{TITRE V : DISPOSITIONS DIVERSES}

ARTICLE 141 : Dans le cadre de l'appui technique et financier aux collectivités territoriales, il est créé un fonds permanent pour le développement des collectivités territoriales.

Les missions, l'organisation et le fonctionnement du fonds sont fixés par décret pris en conseil des ministres.

ARTICLE 142 : Le personnel des collectivités territoriales est régi par le régime juridique applicable aux agents des collectivités territoriales.

\section{LIVREIII DES ORGANESET DEL'ADMINISTRATIONDESCOLLECTIVITES TERRITORIALES}

\section{TITRE I : DES ORGANES ET DE L'ADMINISTRATIONDE LA REGION CHAPITRE I : DES ORGANES DE LA REGION}

ARTICLE 143: Les organes de la région sont :

- le conseil régional qui est l'organe délibérant ;

- le président du conseil régional qui est l'organe exécutif. A ce titre il exécute les délibérations du conseil régional.

Le président du conseil régional est assisté d'un premier vice-président et d'un deuxième vice-président.

Le président du conseil régional et le premier vice-président ont l'obligation de résidence dans la région.

Le conseil régional siège au chef-lieu de la région.

\section{SECTION 1 : DU CONSEIL REGIONAL}

\section{PARAGRAPHE 1. : ORGANISATION}

ARTICLE 144: Le conseil régional élit en son sein le président et les vice-présidents. Il est institué au sein du conseil régional trois commissions permanentes :

1) une commission «affaires générales, sociales et culturelles»;

2) une commission "affaires économiques et financières";

3) une commission «environnement et développement local».

Le conseil régional peut créer en cas de besoin, des commissions ad hoc pour des questions spécifiques. Les modalités de fonctionnement des commissions ad hoc sont fixées par délibérations du conseil régional.

Le président et les vice-présidents ne peuvent pas être responsables de commissions. 


\section{PARAGRAPHE 2. : ATTRIBUTIONS}

ARTICLE 145: Le conseil régional définit les orientations en matière de développement dans la région conformément aux grandes orientations nationales.

A cet effet :

- il discute et adopte les plans de développement régional ;

- il règle par ses délibérations, les affaires de la région et donne son avis sur toutes les questions qui lui sont soumises par l'Etat ou par d'autres collectivités.

ARTICLE 146 : Le conseil régional est consulté sur toutes décisions à prendre par d'autres organes et autorités, sur les questions intéressant la région ou engageant sa responsabilité.

ARTICLE 147 : Le conseil régional délibère sur :

1) le budget primitif;

2) le budget supplémentaire ;

3) le compte administratif et le compte de gestion de la région;

4) les taxes et redevances perçues directement au profit de la région dont la perception est autorisée par la loi ;

5) les acquisitions, les aliénations ou les échanges des biens mobiliers ou immobiliers de la région;

6) l'acceptation ou le refus de dons et legs ;

7) les emprunts à contracter par la région;

8) l'attribution de secours ou de subventions ;

9) les indemnités;

10) toutes autres matières pour lesquelles compétence lui est reconnue par les textes en vigueur.

ARTICLE 148 : Le conseil régional contrôle l'action du président du conseil.

ARTICLE 149 : Les actes de l'autorité régionale ne sont soumis à approbation ou à autorisation préalable que dans les cas formellement prévus par la loi.

ARTICLE 150 : Ne sont exécutoires qu'après approbation ou autorisation de l'autorité de tutelle, les délibérations du conseil régional portant sur les matières suivantes :

$\mathbf{1}^{\circ}$ ) Sont soumis à approbation :

a) le budget primitif ;

b) le budget supplémentaire ;

c) les achats publics dans les limites prévues par les textes en vigueur ;

d) le compte administratif et le compte de gestion ;

e) les conventions portant création des structures de concertation et de coopération ;

f) les opérations d'aménagement du territoire.

$\left.2^{\circ}\right)$ Sont soumis à autorisation préalable :

a) les acquisitions, les aliénations ou les échanges de biens immobiliers ;

b) les emprunts dans les limites prévues par les textes en vigueur ;

126 Textes et textes de loi sur la gestion des ressources naturelles au Burkina Faso 
c) l'acceptation de dons et legs grevés de charges ou entraînant des charges pour la région ;

d) les indemnités ;

e) les contrats assortis de contrepartie de l'Etat ;

f) les opérations d'aménagement du territoire ;

g) les plans locaux de développement.

ARTICLE 151 : Les délibérations du conseil régional qui ne sont pas soumises à approbation ou à autorisation, deviennent exécutoires après leur transmission à l'autorité de tutelle, sous réserve du respect des conditions d'entrée en vigueur des actes des autorités territoriales.

ARTICLE 152 : L'approbation ou l'autorisation est donnée par écrit. Elle est toutefois réputée acquise quarante cinq jours à compter de la date de l'accusé de réception délivré par l'autorité de tutelle.

Lorsque l'autorité de tutelle refuse son autorisation préalable, le conseil peut exercer des recours conformément aux textes en vigueur.

Le conseil régional ne peut déléguer ses attributions.

\section{PARAGRAPHE 3 : FONCTIONNEMENT}

ARTICLE 153 : Le conseil régional statue sur toutes les matières dont il est saisi, soit à l'initiative de son président, soit à la demande d'au moins un tiers de ses membres.

ARTICLE 154 : Le conseil régional se réunit en session ordinaire une fois par trimestre.

ARTICLE 155: Le conseil régional peut se réunir en session extraordinaire, soit à l'initiative de son président, soit à la demande d'au moins un tiers des membres du conseil.

ARTICLE 156 :La durée des sessions ne saurait excéder cinq jours pour les sessions ordinaires et trois jours pour les sessions extraordinaires.

ARTICLE 157 : Les sessions sont convoquées par le président du conseil régional.

Les convocations du conseil régional doivent être adressées par écrit, par affichage et par communiqué aux membres du conseil au moins dix jours francs avant la date fixée pour l'ouverture de la session ordinaire et cinq jours francs avant la date fixée pour la session extraordinaire.

Les convocations doivent comporter l'indication de l'ordre du jour, de la date, de l'heure et du lieu.

A toute convocation doivent être joints tous les documents nécessaires afférents à la tenue de la session.

ARTICLE 158 : Les employeurs sont tenus, au vu de la convocation régulière, de libérer leurs salariés membres du conseil régional, le temps nécessaire pour participer aux sessions du conseil ou aux réunions des commissions spécialisées.

Le temps passé par les salariés aux différentes sessions ou réunions est payé par l'employeur comme temps de travail, sur présentation d'une attestation de présence ou d'un ordre de réquisition dûment signé par le président du conseil. 
ARTICLE 159 : Le conseil régional ne peut valablement siéger que si les deux tiers des membres sont présents à l'ouverture de la session.

Si le quorum n'est pas atteint, la séance est reportée à une date ultérieure qui ne peut excéder sept jours.

A cette seconde séance, la majorité absolue suffit. Si la majorité absolue n'est pas constatée, le Président du conseil régional est tenu dans un délai de sept jours, d'adresser un rapport à l'autorité de tutelle.

L'autorité de tutelle dispose d'un délai de trente jours pour réagir.

Un membre du conseil régional peut se faire représenter par un autre membre. La procuration n'est valable que pour une session.

$\mathrm{Nul}$ ne peut recevoir plus d'une procuration à la fois.

ARTICLE 160 : Les absences non motivées aux sessions donnent lieu à des sanctions qui sont échelonnées comme suit :

- l'avertissement ;

- le blâme.

Un décret portant règlement intérieur-type des conseils de collectivités territoriales précise les modalités d'application des sanctions.

ARTICLE 161 : Les délibérations du conseil régional sont prises à la majorité des membres présents.

Le vote se fait au scrutin secret ou à main levée.

En cas de partage égal des voix, la délibération n'est pas adoptée.

ARTICLE 162 : Les séances du conseil régional sont publiques. Le conseil peut décider à la majorité simple des membres présents, de siéger à huis clos sur tout ou partie de son ordre du jour.

ARTICLE 163 : Les délibérations auxquelles auraient pris part les membres du conseil intéressés, soit en leur nom personnel, soit comme mandataires à l'affaire qui en fait l'objet sont susceptibles d'annulation.

Toute personne ayant intérêt à l'annulation dispose d'un délai de deux mois à compter de la date de publication des délibérations, pour déposer une requête adressée au président du conseil régional.

Il en est donné récépissé.

ARTICLE 164 : La nullité de droit est constatée par l'autorité de tutelle.

ARTICLE 165 : Le président préside le conseil. Il assure la police des séances.

Dans les séances où le compte administratif du président est débattu, le conseil élit un président de séance.

Dans ce cas, le président du conseil peut, même quand il ne serait plus en fonction, assister à la discussion, mais doit se retirer au moment du vote.

ARTICLE 166 : Au début de chaque session et pour sa durée, le conseil régional nomme un ou plusieurs de ses membres pour assurer les fonctions de secrétaire.

Il peut leur adjoindre des auxiliaires pris en dehors de ses membres. Ceux-ci assistent aux séances sans participer aux débats.

128 Textes et textes de loi sur la gestion des ressources naturelles au Burkina Faso 
ARTICLE 167 : Les délibérations du conseil régional sont transcrites par ordre chronologique sur un registre coté et paraphé par l'autorité de tutelle et tenu au siège du conseil.

ARTICLE 168 : Les délibérations du conseil régional sont portées à la connaissance du public par voie d'affichage dans les locaux du conseil régional.

ARTICLE 169 : Si le conseil régional ne se réunit pas ou se sépare avant d'avoir émis un vote sur les questions qui lui sont obligatoirement soumises, le président en informe le gouverneur.

ARTICLE 170 : Le gouverneur est toujours tenu informé par écrit des dates de réunion du conseil régional dans les mêmes délais que les conseillers régionaux et reçoit les procès-verbaux des délibérations.

Il reçoit ampliation de l'ensemble des actes pris par le conseil au plus tard dix jours suivant la fin de la session.

Dans les mêmes conditions, il reçoit ampliation de tout acte pris par le président du conseil régional.

ARTICLE 171 : Au cours du premier trimestre de chaque année, le président du conseil régional rend compte au conseil par un rapport spécial de :

1) la situation de la région sur les matières transférées ;

2) l'activité et du fonctionnement des différents services de la région et des organismes relevant de celle-ci ;

3) l'état d'exécution des délibérations du conseil ;

4) la situation financière de la région.

Ce rapport donne lieu à débats mais n'est pas suivi de vote. La séance est publique et le rapport est transmis au gouverneur pour information.

Nonobstant les dispositions ci-dessus, le conseil régional a obligation de rendre régulièrement compte aux populations de ses activités et de la vie de la région.

\section{PARAGRAPHE 4. : DISSOLUTION DU CONSEIL REGIONAL}

ARTICLE 172 : Lorsque le fonctionnement d'un conseil se révèle impossible, sa dissolution peut être prononcée par décret pris en conseil des ministres, sur rapport du ministre chargé des collectivités territoriales.

Le parlement en est informé par le gouvernement dans un délai de trente jours.

La dissolution ne peut jamais être prononcée par voie de mesure générale.

ARTICLE 173 : En cas de dissolution du conseil, de démission de tous ses membres en exercice ou d'annulation devenue définitive de l'élection de tous ses membres, le gouverneur est chargé de l'expédition des affaires courantes.

Il est procédé à l'élection d'un nouveau conseil régional dans un délai maximum de soixante jours.

Si la dissolution ou la démission de tous les membres du conseil régional survient au cours de la dernière année du mandat, il est procédée à l'installation d'une délégation spéciale présidée par le représentant de l'Etat, dans les conditions fixées par décret pris en conseil des ministres. 
ARTICLE 174 : Le gouverneur convoque les conseillers élus pour la première réunion, dont il fixe le jour, l'heure et le lieu. La date de la réunion ne saurait excéder quinze jours après la proclamation définitive des résultats des élections.

\section{SECTION 2 : DU PRESIDENT ET DES VICE-PRESIDENTS DU CONSEIL REGIONAL}

PARAGRAPHE 1 : ELECTION DU PRESIDENT ET DES VICE-PRESIDENTS

ARTICLE 175 : Le conseil régional élit le président et les vice-présidents parmi ses membres.

Lélection du président et des vice-présidents est faite conformément aux dispositions du code électoral en vigueur.

\section{PARAGRAPHE 2 : ATTRIBUTIONS DU PRESIDENT DU CONSEIL REGIONAL}

ARTICLE 176: Le président du conseil régional est chargé de l'exécution des décisions du conseil.

ARTICLE 177 : Le président du conseil régional exécute les délibérations du conseil régional.

Il est chargé sous le contrôle du conseil de :

1) conserver et administrer le patrimoine de la région;

2) exercer la maitrise d'ouvrage des travaux de la région;

3) prendre les mesures relatives à la voirie régionale ;

4) représenter la région dans les actes de la vie civile;

5) représenter la région en justice ;

6) veiller à l'exécution des programmes de développement ;

7) veiller à la protection de l'environnement et prendre en conséquence les mesures propres, d'une part à empêcher ou à supprimer les atteintes au cadre de vie et d'autre part à assurer la conservation et la gestion durable des ressources naturelles renouvelables de la région.

ARTICLE 178 : Le président du conseil régional représente la région dans les conseils, commissions et organisations dans lesquels cette représentation est prévue par les lois et règlements en vigueur.

ARTICLE 179 : Le président du conseil régional est l'ordonnateur du budget de la région.

Le président du conseil régional est officier de police judiciaire.

PARAGRAPHE 3 : ATTRIBUTIONS DES VICE-PRESIDENTS

ARTICLE 180 : Dès sa prise de fonction le président du conseil régional a obligation de déléguer sous sa responsabilité, par arrêté une partie de ses attributions au profit de chacun des deux vice-présidents.

ARTICLE 181 : Les vice-présidents assurent l'intérim du président du conseil dans l'ordre de leur énumération.

L'intérim est constaté, sauf cas de force majeure, par arrêté du président du conseil.

130 Textes et textes de loi sur la gestion des ressources naturelles au Burkina Faso 


\section{PARAGRAPHE 4: DISPOSITIONS PARTICULIERES APPLICABLES AU PRESIDENT ET AUX VICE-PRESIDENTS DU CONSEIL REGIONAL}

ARTICLE 182 : Le président ou le vice-président nommé à une fonction incompatible avec son mandat de président ou de vice-président est tenu de faire une déclaration d'option dans un délai de trente jours.

Passé ce délai, il est démis d'office de son mandat de président ou de vice - président par l'autorité de tutelle.

ARTICLE 183 : Le président ou le vice-président du conseil régional qui, pour une cause antérieure à son élection ou découverte après celle-ci, ne remplit plus les conditions requises pour être élu, ou qui se trouve dans un des cas d'incompatibilité prévus par le code électoral, doit cesser immédiatement ses fonctions.

Si le président ou le vice-président refuse de démissionner, il est procédé à sa révocation d'office.

ARTICLE 184 : Le président ou le vice-président peut faire l'objet de révocation en cas de faute grave.

Peuvent entraîner la révocation, les fautes graves suivantes :

1) détournement de biens et/ou de deniers publics ;

2) concussion ou corruption ;

3) prêts irréguliers d'argent sur les fonds de la région;

4) faux en écriture publique et usage de faux ;

5) endettement de la région résultant d'une faute de gestion ;

6) refus de signer et/ou de transmettre à l'autorité de tutelle une délibération du conseil régional ;

7) refus de réunir le conseil régional conformément aux textes en vigueur ;

8) spéculation sur l'affectation des terrains publics, les lotissements, les attributions de parcelles, les permis de construire ;

9) absence du président du conseil régional de la région depuis plus de six mois pour des raisons autres que celles relatives à l'intérêt de la collectivité territoriale ou de santé ;

10) poursuite devant un tribunal répressif ou condamnation pour des faits et actes punis par la loi, à l'exception des contraventions de simple police ou des délits d'imprudence, hormis les cas de délit de fuite concomitant.

En tout état de cause, le président ou le vice-président, prévenu des fautes graves cidessus énumérées peut faire l'objet de suspension préalable prononcée par l'autorité de tutelle.

ARTICLE 185 : Il peut être introduit à l'encontre du président du conseil régional une motion de défiance. Pour être recevable, la motion de défiance doit être signée par au moins un tiers des membres du conseil régional. La motion de défiance est déposée auprès du président du conseil régional qui en délivre accusé de réception dans un délai de deux jours ouvrables. Une copie de la motion de défiance est adressée au gouverneur de région dans le même délai.

La motion de défiance doit être motivée. 
ARTICLE 186 : La motion de défiance donne obligatoirement lieu à la tenue d'une session extraordinaire dans les sept jours qui suivent la date de dépôt de la motion. En cas de refus du président du conseil régional, la convocation du conseil est assurée par l'autorité de tutelle. Dans ce cas, la session est présidée par un vice-président.

ARTICLE 187: La motion de défiance ne peut être adoptée qu'au terme d'un débat contradictoire suivi de vote. Elle est acquise à la majorité des deux tiers du conseil régional.

En cas de rejet, une nouvelle motion pour les mêmes motifs ne peut être déposée avant un délai d'un an.

ARTICLE 188 : Le procès-verbal de la séance au cours de laquelle la motion de défiance a été adoptée est transmis au ministre chargé des collectivités territoriales dans un délai maximum de dix jours pour compter de la date de tenue de la session.

ARTICLE 189 : La démission du président du conseil régional pour cause de défiance est constatée par décret pris en conseil des ministres.

Il est procédé dans un délai maximum de trente jours, à l'élection d'un nouveau président du conseil régional.

ARTICLE 190 : Nonobstant les dispositions des articles 184 et 189 ci-dessus, il peut être mis fin aux fonctions du président du conseil régional et/ou de ses vice-présidents en cas de maladie prolongée de plus d'un an et les rendant inaptes à l'exercice des fonctions.

ARTICLE 191 : Toute suspension ou révocation du président ou d'un vice-président doit être précédée d'une audition de l'intéressé ou d'une invitation à fournir des explications par écrit dans les délais requis.

La suspension est prise à titre de mesure conservatoire ; elle relève de l'autorité de tutelle.

La révocation est décidée par décret pris en conseil des ministres.

ARTICLE 192 : Toute décision de l'autorité de tutelle portant suspension ou révocation d'un président ou d'un vice-président du conseil régional est susceptible de recours pour excès de pouvoir devant la juridiction administrative.

ARTICLE 193 : La démission du président du conseil régional est adressée au gouverneur par lettre recommandée avec accusé de réception ; elle est définitive à partir de la date du dépôt de l'acceptation sur le bureau du président.

Le président démissionnaire continue d'exercer ses fonctions jusqu’à la nomination d'un intérimaire.

ARTICLE 194 : En cas de suspension, le président est provisoirement remplacé par un vice-président désigné par l'autorité de tutelle dans l'ordre d'énumération.

A défaut d'un vice-président, l'intérimaire est désigné parmi les conseillers régionaux.

Dès sa nomination, l'intérimaire doit résider effectivement dans la région.

ARTICLE 195 : En cas de décès, de démission, de révocation ou de tout autre empêchement absolu devenu définitif, il est procédé à l'élection d'un nouveau président dans un délai de trente jours.

En attendant cette élection, la conduite des activités de la région est assurée dans les conditions suivantes :

132 Textes et textes de loi sur la gestion des ressources naturelles au Burkina Faso 
- dans les cas de vacance du poste provoquée par le décès du président du conseil régional, le premier vice-président ou à défaut le deuxième vice-président, est d'office chargé de l'intérim ;

- dans les autres cas de vacance du poste de président du conseil régional, il est procédé, conformément aux dispositions de l'article 194 ci-dessus, à la nomination d'un intérimaire dans un délai de sept jours à compter de la date de constatation de la vacance.

ARTICLE 196 : Lorsque le président du conseil régional est révoqué, démis ou suspendu, son remplaçant exerce la plénitude de ses fonctions.

En cas d'absence ou d'empêchement, l'intérimaire est uniquement chargé de l'expédition des affaires courantes.

ARTICLE 197 : La démission des vice-présidents est adressée au président du conseil, par lettre recommandée avec accusé de réception; elle est définitive à partir de la date de l'acceptation de la démission par le président; ils continuent d'exercer leurs fonctions jusqu'à l'installation de leurs successeurs qui doit intervenir dans un délai maximum de quinze jours.

ARTICLE 198 : En cas de décès, de démission, de révocation ou d'empêchement absolu d'un vice-président, il est procédé à son remplacement dans les formes prévues par la loi.

\section{SECTION3:DISPOSITIONSRELATIVESAL'ELECTIONDESCONSEILLERS REGIONAUX}

ARTICLE 199: L'élection des conseillers régionaux est faite conformément aux dispositions du code électoral.

La durée du mandat est de cinq ans.

\section{CHAPITRE II : L'ADMINISTRATION DE LA REGION SECTION 1 : DISPOSITIONS GENERALES}

ARTICLE 200 : Le président du conseil est le chef de l'administration de la collectivité territoriale. A ce titre, il administre toutes les affaires du ressort de la collectivité territoriale et organise les services à caractère administratif, industriel et commercial, aux fins de sauvegarder les intérêts de la collectivité et promouvoir le domaine public et privé de la collectivité.

ARTICLE 201 : Le président du conseil régional est assisté dans ses fonctions administratives par un secrétaire général.

ARTICLE 202: Le secrétaire général est nommé par le président du conseil parmi les cadres supérieurs de l'administration générale.

Le secrétaire général peut être un agent recruté par la région ou un agent mis à disposition ou en position de détachement par l'Etat.

ARTICLE 203 : Le secrétaire général est chargé, sous l'autorité du président du conseil régional :

1) de la coordination administrative et technique des services de la collectivité territoriale;

2) de la gestion du personnel et du matériel de la collectivité ;

3) des relations techniques du conseil régional avec les représentants de l'Etat et les services communaux. 
ARTICLE 204: Outre les attributions énumérées à l'article 203 ci-dessus, le secrétaire général peut recevoir délégation de signature du président du conseil régional.

Les matières dans lesquelles le secrétaire général peut recevoir délégation de signature sont précisées par le conseil régional dans le respect des textes en vigueur et notamment le décret portant règlement intérieur-type des conseils des collectivités territoriales.

\section{SECTION 2 : DU PERSONNEL REGIONAL}

ARTICLE 205 : Le personnel régional est soumis aux dispositions du régime juridique applicable aux agents des collectivités territoriales.

\section{SECTION 3 : DU PATRIMOINE DE LA REGION}

ARTICLE 206 : La région peut créer ou acquérir des biens meubles ou immeubles aux fins d'assurer son fonctionnement ou de soutenir son action dans les domaines économique, social et culturel.

ARTICLE 207 : La propriété de la région peut aussi résulter de dons et legs, de mutations de biens appartenant à l'Etat, à d'autres personnes morales ou physiques de droit public ou privé.

ARTICLE 208 : La région peut être propriétaire de rentes sur l'Etat, notamment, par l'achat de titres ou par l'emploi de capitaux provenant de remboursements faits par des particuliers, d'aliénations, de soultes d'échanges, de legs et donations.

Le placement en rentes sur l'Etat s'opère en vertu d'une délibération du conseil régional.

ARTICLE 209 : Le conseil régional délibère sur le mode d'acquisition, d'aliénation ou de gestion des biens et sur les opérations immobilières effectuées par la région.

ARTICLE 210 : Lorsqu'au moment de sa création, une région ne possède pas de biens propres, l'Etat met à sa disposition les moyens nécessaires au fonctionnement des services régionaux.

L'Etat peut céder à la région les biens lui appartenant et situés dans le territoire régional.

ARTICLE 211 : Les baux, les accords amiables et conventions de toute nature ayant pour objet la prise en location, l'échange d'immeubles ou de droits immobiliers sont conclus après délibération du conseil régional.

\section{SECTION 4 : DES ACTES DU PRESIDENT DU CONSEIL REGIONAL}

ARTICLE 212: Le président du conseil régional exerce son pouvoir réglementaire par voie d'arrêté.

ARTICLE 213 : Les actes du président du conseil régional ne sont exécutoires et/ ou opposables aux tiers qu’après avoir été portés à la connaissance des intéressés par voie de publication ou d'affichage, toutes les fois qu'ils contiennent des dispositions générales et dans les autres cas, par voie de notification individuelle.

ARTICLE 214 : Tout citoyen peut ester en justice contre les actes du président du conseil régional lui faisant grief.

\section{SECTION 5. : DE L'ACTION EN JUSTICE}

ARTICLE 215 : Le conseil régional délibère sur les actions en justice à intenter ou à soutenir par la région. Cependant, le président du conseil régional peut, en cas d'urgence, sans l'autorisation préalable du conseil régional, intenter les actions 
possessoires et accomplir les actes nécessaires à la conservation des droits de la région.

ARTICLE 216 : Le président du conseil régional représente la région en justice. Il peut, sans délibération préalable du conseil régional, poser tous les actes conservatoires.

\section{TITRE II : DES ORGANES ET DE LADMINISTRATION DE LA COMMUNE}

ARTICLE 217 : La commune urbaine est divisée en secteurs et le cas échéant en villages.

La loi détermine les limites territoriales de la commune urbaine.

ARTICLE 218 : La commune rurale comprend un espace d'habitation, un espace de production et un espace de conservation.

La commune rurale est divisée en secteurs et en villages. Le découpage en secteurs est constaté par arrêté du ministre chargé de l'administration du territoire.

L'érection d'agglomérations en villages est constatée par arrêté du ministre chargé de l'administration du territoire, sur proposition du maire de la commune.

La loi détermine les limites territoriales de la commune rurale.

\section{CHAPITRE 1 : DES ORGANES DE LA COMMUNE}

ARTICLE 219 : Les organes de la commune urbaine et de la commune rurale sont :

- le conseil municipal qui est l'organe délibérant;

- le maire qui est l'organe exécutif; à ce titre, il exécute les délibérations du conseil municipal.

Le maire est assisté d'un premier adjoint et d'un deuxième adjoint.

Dès sa prise de fonction, le maire a obligation de déléguer sous sa responsabilité, par arrêté une partie de ses attributions au profit de chacun des deux adjoints.

Le siège de la commune est la mairie.

\section{SECTION 1 : DU CONSEIL MUNICIPAL}

ARTICLE 220 : Le conseil municipal est élu conformément aux dispositions du code électoral.

La durée du mandat du conseil municipal est de cinq ans.

\section{PARAGRAPHE 1 : ORGANISATION}

ARTICLE 221 : Le conseil municipal élit en son sein le maire et les adjoints au maire.

Il est institué au sein du conseil municipal trois commissions permanentes:

1) une commission «affaires générales, sociales et culturelles»;

2) une commission "affaires économiques et financières";

3) une commission «environnement et développement local».

Le conseil municipal peut créer des commissions ad hoc pour des questions spécifiques. Les modalités de fonctionnement des commissions ad hoc sont fixées par délibérations du conseil municipal.

Le maire et ses adjoints ne peuvent pas présider ces commissions.

ARTICLE 222: Dans les communes rurales, il est institué dans chaque village autre que 
le chef-lieu un conseil villageois de développement (CVD). Le conseil de la collectivité territoriale supervise la mise en place des conseils villageois de développement (CVD) dans les villages.

ARTICLE 223 : Le conseil villageois de développement est chargé sous l'autorité du conseil municipal de :

1) contribuer à l'élaboration et à la mise en ouvre des plans communaux de développement ;

2) contribuer à la promotion du développement local dans le village ;

3 participer aux activités des différentes commissions mises en place par le conseil municipal pour la gestion et la promotion du développement local.

Les membres du conseil municipal ne peuvent être membres dirigeants des conseils villageois de développement.

L'organisation, la composition et le fonctionnement des conseils villageois de développement sont fixés par décret pris en conseil des ministres.

\section{PARAGRAPHE 2 : ATTRIBUTIONS}

ARTICLE 224 : Le conseil municipal définit les grandes orientations en matière de développement communal.

Il discute et adopte les plans de développement communaux et contrôle leur exécution.

Il règle par ses délibérations, les affaires de la commune et donne son avis sur toutes les questions qui lui sont soumises par l'Etat ou par d'autres collectivités territoriales.

ARTICLE 225 : Le conseil municipal est consulté sur toutes les décisions à prendre par d'autres organes et autorités sur des questions intéressant la commune ou engageant sa responsabilité.

ARTICLE 226: Le conseil municipal délibère sur :

1) le budget primitif ;

2) le budget supplémentaire ;

3) le compte administratif et le compte de gestion de la commune;

4) les taux des taxes et redevances perçues directement au profit de la commune, ainsi que le taux des centimes additionnels dont la perception est autorisée par la loi ;

5) les acquisitions, les aliénations ou les échanges des biens mobiliers ou immobiliers de la commune ;

6) l'acceptation ou le refus de dons et legs;

7) les emprunts à contracter par la commune ;

8) l'attribution de secours ou de subventions ;

9) les indemnités.

ARTICLE 227 : Le conseil municipal contrôle l'action du maire.

Il contrôle l'exécution des plans de développement communaux et en assure l'évaluation périodique.

ARTICLE 228 : Les actes des autorités municipales ne sont soumis à approbation ou à autorisation préalable, que dans les cas formellement prévus par la loi.

ARTICLE 229 : Ne sont exécutoires qu'après approbation ou autorisation de l'autorité de tutelle, les délibérations du conseil municipal portant sur les matières suivantes:

136 Textes et textes de loi sur la gestion des ressources naturelles au Burkina Faso 
1) Sont soumis à approbation :

a) le budget primitif;

b) le budget supplémentaire;

c) les achats publics dans les limites prévues par les textes en vigueur;

d) le compte administratif et le compte de gestion;

e) les conventions portant création des structures de concertation et de coopération.

2) Sont soumis à autorisation préalable :

a) les acquisitions, les aliénations ou les échanges de biens immobiliers;

b) les emprunts dans les limites prévues par les textes en vigueur;

c) l'acceptation de dons et legs grevés de charges ;

d) les indemnités ;

e) les opérations d'aménagement du territoire ;

f) les contrats assortis de contrepartie de l'Etat ;

g) la mise en œuvre des plans locaux de développement.

ARTICLE 230 : Les délibérations du conseil municipal qui ne sont pas soumises à approbation ou à autorisation deviennent exécutoires après leur transmission à l'autorité de tutelle sous réserve du respect des conditions d'entrée en vigueur des actes des autorités territoriales.

ARTICLE 231 : L'approbation ou l'autorisation est donnée par écrit. Elle est toutefois réputée acquise trente jours à partir de la date de l'accusé de réception délivré par l'autorité de tutelle. Lorsque l'autorité de tutelle refuse son autorisation préalable, le conseil peut exercer des recours conformément aux textes en vigueur.

Le conseil municipal ne peut déléguer ses attributions.

\section{PARAGRAPHE 3 : FONCTIONNEMENT}

ARTICLE 232 : Le conseil municipal statue sur toutes les matières dont il est saisi, soit sur proposition du maire, soit à l'initiative d'au moins un tiers de ses membres.

ARTICLE 233 : Le conseil municipal se réunit en session ordinaire une fois par trimestre sur convocation du maire.

ARTICLE 234 : Le conseil municipal peut se réunir en session extraordinaire sur convocation du maire, soit à son initiative, soit à la demande d'un président de commission permanente, soit d'au moins un tiers des membres du conseil.

ARTICLE 235 : La durée des sessions ne saurait excéder cinq jours pour les sessions ordinaires et trois jours pour les sessions extraordinaires.

ARTICLE 236 : Les convocations du conseil municipal doivent être adressées par écrit, par affichage et par communiqué aux membres du conseil, au moins cinq jours francs avant la date fixée pour l'ouverture de la session ordinaire et deux jours francs avant la date fixée pour l'ouverture de la session extraordinaire.

Les convocations doivent comporter l'indication de l'ordre du jour, de la date, de l'heure et du lieu.

L'autorité administrative territorialement compétente est toujours tenue informée par écrit des dates de réunion du conseil municipal, dans les mêmes délais que les 
conseillers municipaux et reçoit les procès-verbaux des délibérations. Elle reçoit ampliation de l'ensemble des actes pris par le conseil au plus tard dix jours suivant la fin de la session.

Dans les mêmes conditions, elle reçoit ampliation de tout acte pris par le maire.

A toute convocation, doivent être joints tous les documents afférents à la tenue de la session.

ARTICLE 237 : Les employeurs sont tenus, au vu de la convocation régulière, de libérer leurs salariés membres du conseil municipal, le temps nécessaire pour participer aux sessions du conseil ou aux réunions des commissions.

Le temps passé par les salariés aux différentes sessions ou réunions est payé par l'employeur comme temps de travail, sur présentation d'une attestation de présence ou d'un ordre de réquisition dûment signé par le maire.

ARTICLE 238 : Le conseil municipal ne peut valablement siéger que si les deux tiers des membres sont présents à l'ouverture de la session.

Si le quorum n'est pas atteint, la séance est reportée à une date ultérieure qui ne peut excéder sept jours.

A cette seconde séance, la majorité absolue suffit. Si la majorité absolue n'est pas constatée, le président du conseil municipal est tenu, dans un délai de sept jours, d'adresser un rapport à l'autorité de tutelle.

L'autorité de tutelle dispose d'un délai de trente jours pour réagir.

ARTICLE 239 : Les absences non motivées aux sessions donnent lieu à des sanctions qui sont échelonnées comme suit :

- l'avertissement ;

- le blâme.

Un décret portant règlement intérieur-type des conseils de collectivités territoriales précise les modalités d'application des sanctions.

ARTICLE 240 : Les délibérations du conseil municipal sont prises à la majorité des membres présents.

Le vote se fait au scrutin secret ou à main levée.

En cas de partage égal des voix, la délibération n’est pas adoptée.

ARTICLE 241 : Les séances du conseil municipal sont publiques. Le conseil peut décider à la majorité simple des membres présents, de siéger à huis clos sur tout ou partie de son ordre du jour.

ARTICLE 242 : Les délibérations auxquelles auraient pris part les membres du conseil intéressés, soit en leur nom personnel, soit comme mandataires à l'affaire qui en fait l'objet sont susceptibles d'annulation.

Toute personne intéressée dispose d'un délai de deux mois à compter de la date de publication des délibérations, pour déposer une requête adressée au maire.

Il en est donné récépissé.

ARTICLE 243 : La nullité de droit est constatée par l'autorité de tutelle.

ARTICLE 244 : Le maire préside les séances du conseil municipal.

Il assure la police des séances.

138 Textes et textes de loi sur la gestion des ressources naturelles au Burkina Faso 
Dans les séances où le compte administratif du maire est débattu, le conseil élit un président de séance.

Dans ce cas, le maire peut, même quand il ne serait plus en fonction, assister à la discussion, mais doit se retirer au moment du vote.

ARTICLE 245 : Au début de chaque session et pour sa durée, le conseil municipal nomme un ou plusieurs de ses membres, pour assurer les fonctions de secrétaire.

Il peut leur adjoindre des auxiliaires pris en dehors de ses membres. Ceux-ci assistent aux séances sans participer aux débats.

ARTICLE 246 : Les délibérations du conseil municipal sont transcrites par ordre chronologique sur un registre coté et paraphé par l'autorité de tutelle et tenu au siège du conseil.

ARTICLE 247 : Les délibérations du conseil municipal sont portées à la connaissance du public par voie d'affichage dans les locaux de la mairie et en tout autre lieu approprié.

ARTICLE 248 : Si le conseil municipal ne se réunit pas ou se sépare avant d'avoir émis un vote sur les questions qui lui sont obligatoirement soumises, le maire en informe l'autorité de tutelle.

ARTICLE 249 : L'autorité de tutelle est toujours tenue informée par écrit, des dates de réunion du conseil municipal et reçoit les procès-verbaux des délibérations.

ARTICLE 250 : Au cours du premier trimestre de chaque année, le maire rend compte au conseil municipal par un rapport spécial de:

1) la situation de la commune sur les matières transférées;

2) l'activité et du fonctionnement des différents services de la commune et des organismes relevant de celle-ci ;

3) l'état d'exécution des délibérations du conseil ;

4) la situation financière de la commune urbaine.

Ce rapport donne lieu à débats mais n'est pas suivi de vote. La séance est publique et le rapport est transmis à l'autorité administrative territorialement compétente pour information.

\section{PARAGRAPHE 4 : DISSOLUTION DU CONSEIL MUNICIPAL}

ARTICLE 251: Lorsque le fonctionnement d'un conseil municipal se révèle impossible, sa dissolution peut être prononcée par décret pris en conseil des ministres sur rapport du ministre chargé des collectivités territoriales.

La dissolution ne peut jamais être prononcée par voie de mesure générale.

ARTICLE 252 : En cas de dissolution du conseil municipal, de démission de tous ses membres en exercice ou d'annulation devenue définitive de l'élection de tous ses membres, le représentant de l'Etat territorialement compétent est chargé de l'expédition des affaires courantes.

Il est procédé à la réélection du conseil municipal dans un délai maximum de soixante jours.

Si la dissolution ou la démission de tous les membres du conseil municipal survient au cours de la dernière année du mandat, il est procédé à l'installation d'une délégation 
spéciale présidée par le représentant de l'Etat, dans les conditions fixées par décret pris en conseil des ministres.

ARTICLE 253 : La démission du ou des conseillers est adressée au maire.

ARTICLE 254 : Le représentant de l'Etat territorialement compétent convoque les conseillers élus pour la première réunion, dont il fixe le jour, l'heure et le lieu. La date de la réunion ne saurait excéder sept jours après la proclamation définitive des résultats.

\section{SECTION 2. : DU MAIRE ET DES ADJOINTS AU MAIRE}

\section{PARAGRAPHE 1 : ELECTION DU MAIRE ET DES ADJOINTS AU MAIRE}

ARTICLE 255 : Le conseil municipal élit le maire et les adjoints au maire parmi ses membres.

Lélection du maire et de ses adjoints se fait conformément aux dispositions du code électoral.

\section{PARAGRAPHE 2 : ATTRIBUTIONS DU MAIRE}

ARTICLE 256: Le maire est chargé de l'exécution des décisions du conseil municipal.

ARTICLE 257 : Le maire est ordonnateur du budget communal.

ARTICLE 258 : Le maire est chargé, sous le contrôle du conseil municipal de :

1) conserver et administrer les propriétés de la commune ;

2) exercer la maîtrise d'ouvrage des travaux de la commune;

3) prendre les mesures relatives à la voirie municipale ;

4) représenter la commune dans les actes de la vie civile ;

5) représenter la commune en justice ;

6) veiller à l'exécution des programmes de développement ;

7) veiller à la protection de l'environnement, en prenant d'une part, des mesures propres à empêcher ou à supprimer les atteintes au cadre de vie et d'autre part, à améliorer ce cadre de vie et à assurer la conservation et la gestion durable des ressources naturelles renouvelables de la commune.

ARTICLE 259 : Le maire représente la commune dans les conseils, commissions et organismes dans lesquels cette représentation est prévue par les lois et règlements en vigueur.

ARTICLE 260 : Le maire est chargé de la police municipale ayant pour but d'assurer la sûreté, la sécurité, la salubrité et la tranquillité.

ARTICLE 261: La fonction de police municipale du maire comprend :

1) la protection du domaine et des lieux publics, les mesures de police administrative visant à assurer la commodité de la circulation, la protection des aliénés, l'occupation régulière du domaine public, l'exercice de la profession de marchand fixe ou ambulant;

2) la protection des personnes et de leurs biens ;

3) les mesures visant à assurer la salubrité et l'hygiène publiques et qui concernent les opérations funéraires, le contrôle sanitaire des établissements recevant du public, la lutte contre les fléaux, les calamités et les produits incommodes ou dangereux pour la santé publique; 
4) les mesures visant à assurer le droit à la tranquillité et au repos et qui concernent les mesures contre les bruits, les tapages, les indécences;

5) l'application des mesures prises en matière de divagation des animaux.

ARTICLE 262: Le maire assure la police des routes à l'intérieur du territoire communal, dans les limites des règlements en matière de circulation routière.

A cet effet, il délivre :

- les permis de stationnement ou de dépôt temporaire sur la voie publique et autres lieux publics, sous réserve que cette mesure ne gène pas la circulation ;

- les autorisations d'alignements individuels et de construire et les autres permissions de voirie à titre précaire et essentiellement révocables, ayant pour objet notamment l'établissement dans le sol de la voie publique de canalisations destinées au passage ou à la conduite d'eau, d'électricité, de gaz, du téléphone.

ARTICLE 263 : Le maire est officier d'état civil. Il est responsable du centre principal d'état civil. Il peut créer des centres secondaires d'état civil qui fonctionnent sous son contrôle.

ARTICLE 264 : Le maire est officier de police judiciaire.

\section{PARAGRAPHE 3 : ATTRIBUTIONS DES ADJOINTS AU MAIRE}

ARTICLE 265 : Le maire délègue sous sa responsabilité et par arrêté, une partie de ses attributions à chacun de ses adjoints.

ARTICLE 266 : Le premier adjoint au maire a obligation de résidence dans la commune.

ARTICLE 267 : Les adjoints au maire sont officiers d'état civil et officiers de police judiciaire.

ARTICLE 268 : Les adjoints assurent l'intérim du maire dans l'ordre de leur énumération.

PARAGRAPHE 4: DISPOSITIONS PARTICULIERES APPLICABLES AUX MAIRES ET AUX ADJOINTS

ARTICLE 269 : Le maire ou l'adjoint nommé à une fonction incompatible avec son mandat municipal est tenu de faire une déclaration d'option dans un délai de trente jours. Passé ce délai, il est démis d'office de son mandat de maire ou d'adjoint par l'autorité de tutelle.

ARTICLE 270 : Le maire ou l'adjoint qui, pour une cause antérieure à son élection ou découverte après celle-ci ne remplit plus les conditions requises pour être maire ou adjoint ou qui se trouve dans un des cas d'incompatibilité prévus par le code électoral doit cesser immédiatement ses fonctions.

Si le maire ou l'adjoint refuse de démissionner, il est procédé à sa révocation d'office.

ARTICLE 271 : Toute suspension ou révocation du maire ou d'un adjoint doit être précédée d'une audition de l'intéressé ou d'une invitation à fournir des explications par écrit dans les délais requis. La suspension ne peut excéder trois mois.

La suspension relève de l'autorité de tutelle.

La révocation est décidée par décret pris en conseil des ministres.

ARTICLE 272 : Le maire ou un adjoint peut faire l'objet de suspension ou de révocation en cas de faute grave. 
Peuvent entraîner la révocation prévue à l'alinéa ci-dessus, les fautes graves suivantes:

1) détournement de biens et/ou de deniers publics;

2) concussion et/ou corruption ;

3) prêts irréguliers d'argent sur les fonds de la commune ;

4) faux en écriture publique et usage de faux ;

5) endettement de la commune résultant d'une faute de gestion;

6) refus de signer et/ou de transmettre à l'autorité de tutelle une délibération du conseil municipal ;

7) refus de réunir le conseil municipal conformément aux textes en vigueur ;

8) spéculation sur l'affectation des terrains publics, les lotissements, les attributions de parcelles, les permis de construire ;

9) absence du président du conseil municipal depuis plus de six mois pour des raisons autres que celles relatives à l'intérêt de la collectivité territoriale ou de santé ;

10) poursuite devant un tribunal répressif ou condamnation pour des faits et actes punis par la loi, à l'exception des contraventions de simple police ou des délits d'imprudence, hormis les cas de délit de fuite concomitant.

En tout état de cause, le maire ou l'adjoint prévenu des fautes graves ci-dessus énumérées peut faire l'objet de suspension préalable prononcée par l'autorité de tutelle.

ARTICLE 273 : Il peut être introduit à l'encontre du maire une motion de défiance. Pour être recevable, la motion de défiance doit être signée par au moins un tiers des membres du conseil municipal. La motion de défiance est déposée auprès du maire qui doit en donner accusé de réception dans un délai de deux jours ouvrables. Une copie de la motion de défiance est adressée au haut commissaire dans le même délai.

La motion de défiance doit être motivée.

ARTICLE 274: La motion de défiance donne obligatoirement lieu à la tenue d'une session extraordinaire dans les sept jours qui suivent la date de dépôt de la motion. En cas de refus du maire, la convocation du conseil est assurée par l'autorité de tutelle.

Dans ce cas, la session est présidée par un adjoint au maire.

ARTICLE 275 : La motion de défiance ne peut être adoptée qu'au terme d'un débat contradictoire suivi de vote. Elle est acquise à la majorité des deux tiers du conseil municipal.

En cas de rejet, une nouvelle motion de défiance ne peut être déposée pour les mêmes motifs avant le délai d'un an.

ARTICLE 276 : Le procès-verbal de la séance au cours de laquelle la motion de défiance a été adoptée est transmise au ministre chargé des collectivités territoriales dans un délai maximum de dix jours pour compter de la date de tenue de la session.

ARTICLE 277 : La démission du maire pour cause de défiance est constatée par décret pris en conseil des ministres.

Il est procédé dans un délai maximum de trente jours à l'élection d'un nouveau maire.

ARTICLE 278 : Nonobstant les dispositions de l'article 277 ci-dessus, il peut être mis fin aux fonctions du maire et/ou de ses adjoints, en cas de maladie prolongée de plus d'un an et les rendant inaptes à l'exercice de leurs fonctions.

142 Textes et textes de loi sur la gestion des ressources naturelles au Burkina Faso 
ARTICLE 279: Toute décision de l'autorité de tutelle portant suspension ou révocation du maire ou d'un adjoint est susceptible de recours pour excès de pouvoir devant la juridiction administrative.

ARTICLE 280 : La démission du maire est adressée à l'autorité de tutelle, par lettre recommandée avec accusé de réception; elle est définitive à partir de la date du dépôt de l'acceptation de l'autorité de tutelle sur le bureau du maire.

Le maire démissionnaire continue d'exercer ses fonctions jusqu'à la nomination d'un intérimaire.

ARTICLE 281 : En cas de suspension, le maire est provisoirement remplacé par un intérimaire nommé parmi les adjoints par l'autorité de tutelle dans l'ordre d'énumération.

A défaut d'un adjoint, l'intérimaire est choisi parmi les conseillers municipaux.

Dès sa nomination, l'intérimaire doit résider effectivement dans la commune.

ARTICLE 282 :En cas de décès, de démission, de révocation ou de tout autre empêchement absolu et définitif, il est procédé à l'élection d'un nouveau maire dans un délai maximum de trente jours.

En attendant cette élection, la conduite des activités de la commune est assurée dans les conditions suivantes :

- dans le cas où la vacance du poste est provoquée par le décès du maire, le premier adjoint, ou à défaut le deuxième adjoint, est d'office chargé de l'intérim ;

- dans les autres cas de vacance du poste, il est procédé, dans un délai maximum de sept jours, à la nomination d'un intérimaire conformément aux dispositions de l'article 281 ci-dessus.

ARTICLE 283 : Lorsque le maire est révoqué, démis ou suspendu, son intérimaire exerce la plénitude de ses fonctions.

En cas d'empêchement, l'intérimaire exerce la plénitude des fonctions du maire.

Constituent des cas d'empêchement les situations ci-après :

1) l'invalidité dûment constatée ou l'incapacité totale ou partielle à plus de cinquante pour cent ;

2) l'absence prolongée de plus de six mois pour toute autre raison que celle de l'intérêt de la commune ;

3) toute autre situation dûment constatée et empêchant le maire d'exercer ses fonctions pendant une période d'au moins six mois.

En cas d'absence, l'intérimaire est uniquement chargé de l'expédition des affaires courantes.

ARTICLE 284 : La démission des adjoints au maire est adressée au maire par lettre recommandée avec accusé de réception; elle est définitive à partir de la date de l'acceptation de la démission par l'autorité de tutelle; ils continuent d'exercer leurs fonctions jusqu'à l'installation de leurs successeurs qui doit intervenir dans un délai maximum de quinze jours.

ARTICLE 285 : En cas de décès, de démission, de révocation ou de tout autre empêchement absolu d'un adjoint, il est procédé à son remplacement dans les formes prévues par la loi. 


\section{SECTION 3: DISPOSITIONS RELATIVES A L'ELECTION DES CONSEILLERS DES COMMUNES}

ARTICLE 286 : L'élection des conseillers des communes est faite conformément aux dispositions du code électoral.

La durée du mandat est de cinq ans.

\section{CHAPITRE II : DE L'ADMINISTRATION DE LA COMMUNE SECTION 1 : DISPOSITIONS GENERALES}

ARTICLE 287 : Le maire est le chef de l'administration communale. A ce titre, il administre toutes les affaires du ressort communal et organise les services à caractère administratif, industriel et commercial, aux fins de sauvegarder les intérêts de la commune et de promouvoir le domaine public et privé communal.

ARTICLE 288 : Le maire est assisté dans ses fonctions administratives par un secrétaire général de mairie.

ARTICLE 289: Le secrétaire général est nommé par le maire parmi les agents des catégories $\mathrm{A}, \mathrm{B}$ ou $\mathrm{C}$ de l'administration générale ou agents assimilés.

Le secrétaire général peut être un agent recruté par la commune ou un agent mis à disposition ou en position de détachement par l'Etat.

ARTICLE 290 : Le secrétaire général est chargé, sous l'autorité du maire :

1) de la coordination administrative et technique des services de la mairie ;

2) de la gestion du personnel et du matériel de la commune ;

3) des relations techniques de la mairie avec les services de l'Etat.

ARTICLE 291 : Outre les attributions énumérées à l'article 290 ci-dessus, le secrétaire général peut recevoir délégation de signature du maire.

Les matières dans lesquelles le secrétaire général peut recevoir délégation de signature sont précisées par le conseil municipal, conformément aux textes en vigueur, notamment le décret portant règlement intérieur-type des conseils de collectivités territoriales.

\section{SECTION 2 : DU PERSONNEL COMMUNAL}

ARTICLE 292 : Le personnel communal est soumis aux dispositions de la loi portant régime juridique applicable aux agents des collectivités territoriales.

\section{SECTION 3 : DU PATRIMOINE DE LA COMMUNE}

ARTICLE 293 : La commune peut créer ou acquérir des biens meubles ou immeubles aux fins d'assurer son fonctionnement ou de soutenir son action dans les domaines économique, social, culturel et environnemental.

ARTICLE 294: Lorsqu'au moment de sa création, une commune ne possède pas de biens propres, l'Etat met à sa disposition les moyens nécessaires au fonctionnement des services communaux.

L'Etat peut céder à la commune des biens lui appartenant et situés dans le territoire communal.

ARTICLE 295 : La propriété de la commune peut aussi résulter de dons et legs, de mutations de biens appartenant à l'Etat, à d'autres personnes morales ou physiques de droit public ou privé.

ARTICLE 296 : La commune peut être propriétaire de rentes sur l'Etat, notamment, par l'achat de titres ou par l'emploi de capitaux provenant de remboursements faits par des particuliers, d'aliénations, des soultes d'échanges, de legs et donations.

144 Textes et textes de loi sur la gestion des ressources naturelles au Burkina Faso 
Le placement en rentes sur l'Etat s'opère en vertu d'une délibération du conseil municipal.

ARTICLE 297 : Le conseil municipal délibère sur le mode d'acquisition, d'aliénation ou de gestion des biens et sur les opérations immobilières effectuées par la commune.

ARTICLE 298 : Les baux, les accords amiables et conventions de toute nature ayant pour objet la prise en location, l'échange d'immeubles ou de droits immobiliers sont conclus après délibération du conseil municipal.

\section{SECTION 4 : DES ACTES DU MAIRE}

ARTICLE 299 : Le maire exerce son pouvoir réglementaire par voie d'arrêté.

ARTICLE 300 : Les actes du maire ne sont exécutoires et/ou opposables aux tiers qu'après avoir été portés à la connaissance des intéressés, par voie de publication ou d'affichage, toutes les fois qu'ils contiennent des dispositions générales et dans les autres cas par voie de notification individuelle.

ARTICLE 301 : Tout citoyen peut ester en justice contre les actes du maire lui faisant grief.

\section{SECTION 5 : DE L'ACTION EN JUSTICE}

ARTICLE 302 : Le conseil municipal délibère sur les actions en justice à intenter ou à soutenir par la commune. Cependant, le maire peut, en cas d'urgence, sans l'autorisation préalable du conseil municipal, intenter les actions possessoires et accomplir les actes nécessaires à la conservation des droits de la commune.

ARTICLE 303 : Le maire représente la commune en justice.

Il peut toujours, sans délibération préalable du conseil municipal, poser tous les actes conservatoires.

\section{TITRE III : DISPOSITIONS DIVERSES}

ARTICLE 304 : Le mandat de conseiller régional, les fonctions de président et de vice-président sont gratuits.

Le conseil régional peut voter sur ses ressources ordinaires des indemnités pour frais de représentation au profit de ses membres.

Les montants de ces indemnités doivent être conformes à la grille autorisée par les textes en vigueur.

ARTICLE 305 : Les frais des missions commandées par la région sont à la charge de la région.

ARTICLE 306 : Dans les cas où les intérêts personnels du président du conseil régional ou ceux de ses ascendants, descendants et alliés se trouvent en opposition avec ceux de la région, le conseil régional désigne un de ses membres pour représenter la région dans les matières qu'il détermine.

ARTICLE 307 : Tout membre du conseil régional peut faire l'objet de révocation conformément aux dispositions de l'article 184 de la présente loi.

ARTICLE 308 : Le mandat de conseiller municipal, les fonctions de maire et d'adjoints au maire sont gratuits.

Le conseil municipal peut voter sur les ressources ordinaires du budget communal des indemnités pour frais de représentation au profit de ses membres. 
Les montants de ces indemnités doivent être conformes à la grille autorisée par les textes en vigueur.

ARTICLE 309 : Les frais des missions commandées par la commune sont à la charge de la commune.

ARTICLE 310 : Dans les cas où les intérêts personnels du maire ou ceux de ses ascendants, descendants et alliés se trouvent en opposition avec ceux de la commune, le conseil municipal désigne un de ses membres pour représenter la commune dans les matières qu'il détermine.

ARTICLE 311 : Tout membre du conseil municipal peut faire l'objet de suspension ou de révocation conformément aux dispositions des articles 272 et 278 de la présente loi.

\section{LIVRE IV DES COMMUNES A STATUT PARTICULIER}

\section{TITRE I : DE LADMINISTRATION DE LA COMMUNE A STATUT PARTICULIER}

ARTICLE 312 : L'organe de délibération de la commune à statut particulier est le conseil municipal.

ARTICLE 313 : La commune à statut particulier est administrée par un maire et des adjoints élus au sein du conseil municipal, conformément à la législation en vigueur pour les communes.

ARTICLE 314 :Le conseil municipal est l'instance qui réunit les conseillers des arrondissements de la commune à statut particulier.

ARTICLE 315 :Les conditions et modalités d'élection du conseil municipal, ainsi que ses attributions et fonctionnement sont ceux définis par la législation relative à l'organisation et à l'administration des communes.

ARTICLE 316 : Le maire de la commune à statut particulier exerce les attributions conférées au maire de commune urbaine, sous réserve des dérogations prévues par la loi.

Il est assisté dans ses fonctions par un secrétaire général de mairie. Le secrétaire général de mairie est choisi parmi les agents de la catégorie A de l'administration générale.

\section{TITRE II : DE L'ADMINISTRATION DE L'ARRONDISSEMENT}

ARTICLE 317 :L'arrondissement est constitué de secteurs et peut comprendre des villages.

ARTICLE 318 : Lorgane de délibération de l'arrondissement est le conseil d'arrondissement.

ARTICLE 319 : Les conseillers d'un même arrondissement constituent le conseil d'arrondissement.

ARTICLE 320 : Chaque conseil d'arrondissement élit en son sein un maire d'arrondissement et des adjoints au maire.

ARTICLE 321 : Le maire d'arrondissement exerce certaines attributions conférées au maire de commune urbaine sur l'étendue du territoire de l'arrondissement. Il reçoit délégation de pouvoirs du maire de la commune à statut particulier, après délibération du conseil municipal.

146 Textes et textes de loi sur la gestion des ressources naturelles au Burkina Faso 
Le maire d'arrondissement est assisté dans ses fonctions par un secrétaire général de mairie choisi parmi les personnels de la catégorie A de l'administration générale.

ARTICLE 322 : La composition, les attributions et le fonctionnement du conseil d'arrondissement sont ceux reconnus au conseil municipal par la législation en vigueur relative à l'organisation des communes.

ARTICLE 323 : Chaque arrondissement dispose d'un siège appelé mairie d'arrondissement.

ARTICLE 324 : Chaque arrondissement a la charge de mettre en place un minimum de services à même de résoudre les problèmes immédiats des populations. Ces services concernent notamment :

1) l'état civil;

2) les services sociaux;

3) les régies de recettes.

Chaque arrondissement doit veiller à faire assurer ou respecter la propreté et l'embellissement de la collectivité.

ARTICLE 325 : Le maire d'arrondissement et le conseil d'arrondissement sont compétents pour toutes les affaires particulières ou spécifiques à l'arrondissement, à l'exclusion de toute matière ayant un intérêt général pour tout ou partie de la commune à statut particulier.

ARTICLE 326 : L'arrondissement doit s'attacher à promouvoir le cadre de vie de ses populations par :

1) l'entretien des rues et des caniveaux ;

2) la gestion des marchés des secteurs;

3) l'embellissement ;

4) l'aménagement et/ou la gestion des services sociaux suivants :

a) les crèches et jardins d'enfants ;

b) les maisons des jeunes et de la culture principalement destinées aux habitants de l'arrondissement ;

c) les terrains de jeux et d'éducation physique de secteur ;

d) les stades à l'exception du stade municipal principal et des stades dont la gestion a été confiée à la commune par un texte particulier ;

e) les espaces verts ;

f) tout équipement principalement destiné aux habitants de l'arrondissement, sous réserve de ceux gérés par la commune.

ARTICLE 327 : Le conseil municipal peut, en outre, déléguer au conseil d'arrondissement, avec l'accord de celui-ci, la gestion de tout équipement ou service de la commune. Ces délégations prennent fin de plein droit au prochain renouvellement du conseil municipal.

ARTICLE 328 : Le conseil d'arrondissement est saisi pour avis, dans les délais fixés par le maire, des rapports de présentation et des projets de délibération concernant les affaires dont l'exécution est prévue en tout ou en partie dans les limites de l'arrondissement, avant leur examen par le conseil municipal, sous réserve des règles relatives à l'élaboration du budget. 
ARTICLE 329 : Le conseil d'arrondissement est consulté sur les conditions générales d'admission dans les crèches, les écoles maternelles, les foyers sociaux gérés par la commune.

ARTICLE 330 : Les délibérations du conseil d'arrondissement ne peuvent en aucun cas être contraires aux délibérations du conseil municipal, sous peine de nullité constatée par le maire de la commune à statut particulier. La décision du maire peut faire l'objet de recours devant l'autorité de tutelle.

ARTICLE 331 : Pour l'exécution des attributions visées aux articles 325 et 326, les maires d'arrondissement entretiennent avec le maire de la commune à statut particulier et le conseil municipal des rapports de collaboration et non de hiérarchie ou de tutelle.

ARTICLE 332 : Les arrondissements peuvent entretenir des relations de jumelage et de coopération avec d'autres collectivités territoriales et/ou avec tout autre partenaire, sous réserve de l'approbation du conseil municipal.

ARTICLE 333 : Le maire d'arrondissement peut recevoir délégation de pouvoir du maire de la commune, pour des matières intéressant l'arrondissement, mais relevant de la compétence du maire de la commune à statut particulier.

ARTICLE 334 : Le maire d'arrondissement est consulté sur :

1) toute autorisation d'occupation du sol dans l'arrondissement délivrée par le maire de la commune à statut particulier;

2) tout changement d'affectation d'un immeuble communal situé dans l'arrondissement;

3) l'exercice de tout droit de préemption.

ARTICLE 335 : Le maire d'arrondissement est informé par le maire de la commune, des conditions générales de réalisation des projets d'équipement dont l'exécution est prévue, en tout ou en partie, dans les limites de l'arrondissement.

\section{TITRE III : DES LIMITES DES COMMUNES A STATUT PARTICULIER}

ARTICLE 336 :Les limites des communes à statut particulier sont fixées par la loi. Elles comprennent la description des limites de la commune et de celles des arrondissements qui la composent.

\section{TITRE IV : DISPOSITIONS TRANSITOIRE ET FINALE}

ARTICLE 337 : Les communes à statut particulier existant à l'entrée en vigueur de la présente loi demeurent.

ARTICLE 338 : La présente loi qui abroge toutes dispositions antérieures contraires sera exécutée comme loi de l'Etat.

Ainsi fait et délibéré en séance publique à Ouagadougou, le 21 décembre 2004.

Le Président

Roch Marc Christian KABORE

Le Secrétaire de séance

Saïdou KABORE

148 Textes et textes de loi sur la gestion des ressources naturelles au Burkina Faso 



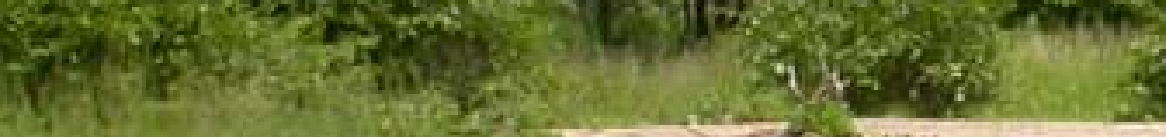

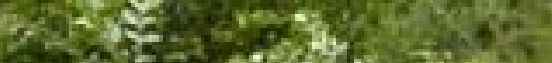

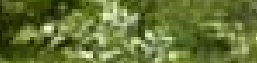

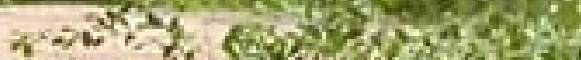

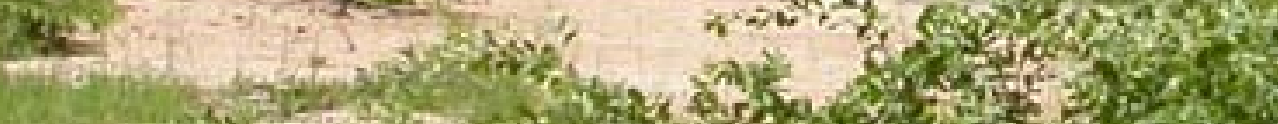

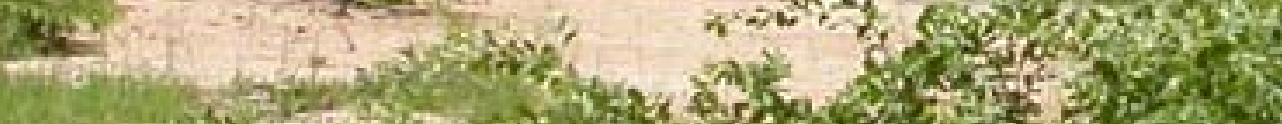

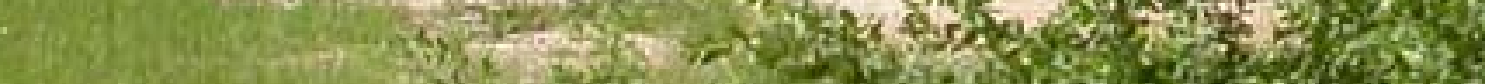

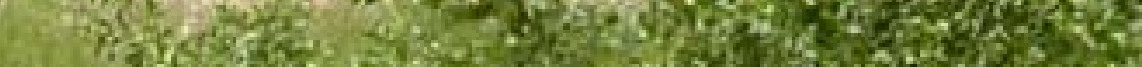

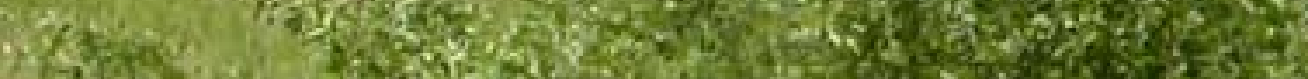

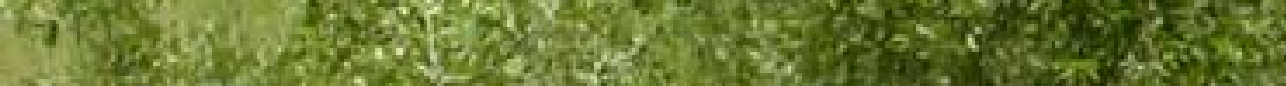
yon 43629

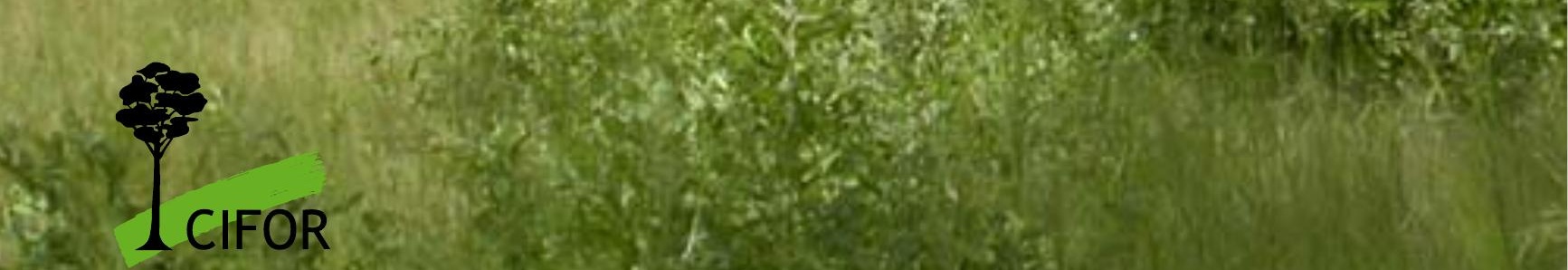

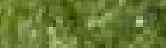

